\title{
THE GREAT OBSERVATORIES ALL-SKY LIRG SURVEY: COMPARISON OF ULTRAVIOLET AND FAR-INFRARED PROPERTIES
}

\author{
Justin H. Howell ${ }^{1}$, Lee Armus ${ }^{1}$, Joseph M. Mazzarella ${ }^{2}$, Aaron S. Evans ${ }^{3,4}$, Jason A. Surace ${ }^{1}$, David B. Sanders ${ }^{5}$, \\ Andreea Petric ${ }^{1}$, Phil Appleton ${ }^{6}$, Greg Bothun ${ }^{7}$, Carrie Bridge ${ }^{1,8}$, Ben H. P. Chan ${ }^{2}$, Vassilis Charmandaris ${ }^{9,10,11}$, \\ David T. Frayer ${ }^{6}$, Sebastian Haan ${ }^{1}$, Hanae Inami ${ }^{1}$, Dong-Chan Kim ${ }^{3}$, Steven Lord ${ }^{6}$, Barry F. Madore ${ }^{2,12}$, \\ Jason Melbourne $^{8}$, Bernhard Schulz ${ }^{6}$, Vivian ${ }^{5,13}$, Tatjana Vavilkin $^{14}$, Sylvain Veilleux ${ }^{15}$, and Kevin Xu ${ }^{6}$ \\ ${ }^{1}$ Spitzer Science Center, MS 220-6, California Institute of Technology, Pasadena, CA 91125, USA; jhhowell@ipac.caltech.edu \\ ${ }^{2}$ Infrared Processing \& Analysis Center, MS 100-22, California Institute of Technology, Pasadena, CA 91125, USA \\ ${ }^{3}$ Department of Astronomy, University of Virginia, P.O. Box 400325, Charlottesville, VA 22904, USA \\ ${ }^{4}$ National Radio Astronomy Observatory, 520 Edgemont Road, Charlottesville, VA 22903, USA \\ ${ }^{5}$ Institute for Astronomy, University of Hawaii, 2680 Woodlawn Drive, Honolulu, HI 96822, USA \\ ${ }^{6}$ NASA Herschel Science Center, IPAC, MS 100-22, California Institute of Technology, Pasadena, CA 91125, USA \\ ${ }^{7}$ Department of Physics, University of Oregon, Eugene, OR 97403, USA \\ ${ }^{8}$ Caltech Optical Observatories, Division of Physics, Mathematics and Astronomy, Mail Stop 320-47, California Institute of Technology, Pasadena, CA 91125, USA \\ ${ }^{9}$ University of Crete, Department of Physics, Heraklion 71003, Greece \\ ${ }^{10} \mathrm{IESL} /$ Foundation for Research and Technology-Hellas, GR-71110, Heraklion, Greece \\ ${ }^{11}$ Chercheur Associé, Observatoire de Paris, F-75014, Paris, France \\ 12 The Observatories, Carnegie Institution of Washington, 813 Santa Barbara Street, Pasadena, CA 91101, USA \\ ${ }^{13}$ Harvard-Smithsonian Center for Astrophysics, 60 Garden St., Cambridge, MA 02138, USA \\ ${ }^{14}$ Department of Physics and Astronomy, State University of New York at Stony Brook, Stony Brook, NY 11794-3800, USA \\ ${ }^{15}$ Department of Astronomy, University of Maryland, College Park, MD 20742, USA \\ Received 2009 August 9; accepted 2010 April 1; published 2010 April 30
}

\begin{abstract}
The Great Observatories All-sky LIRG Survey (GOALS) consists of a complete sample of 202 luminous infrared galaxies (LIRGs) selected from the IRAS Revised Bright Galaxy Sample (RBGS). The galaxies span the full range of interaction stages, from isolated galaxies to interacting pairs to late stage mergers. We present a comparison of the UV and infrared properties of 135 galaxies in GOALS observed by GALEX and Spitzer. For interacting galaxies with separations greater than the resolution of GALEX and Spitzer $\left(\sim 2^{\prime \prime}-6^{\prime \prime}\right)$, we assess the UV and IR properties of each galaxy individually. The contribution of the FUV to the measured star formation rate (SFR) ranges from $0.2 \%$ to $17.9 \%$, with a median of $2.8 \%$ and a mean of $4.0 \% \pm 0.4 \%$. The specific star formation rate (SSFR) of the GOALS sample is extremely high, with a median value $\left(3.9 \times 10^{-10} \mathrm{yr}^{-1}\right)$ that is comparable to the highest SSFRs seen in the Spitzer Infrared Nearby Galaxies Survey sample. We examine the position of each galaxy on the IR excess-UV slope (IRX- $\beta$ ) diagram as a function of galaxy properties, including IR luminosity and interaction stage. The LIRGs on average have greater IR excesses than would be expected based on their UV colors if they obeyed the same relations as starbursts with $L_{\mathrm{IR}}<10^{11} L_{\odot}$ or normal late-type galaxies. The ratio of $L_{\mathrm{IR}}$ to the value one would estimate from the IRX $-\beta$ relation published for lower luminosity starburst galaxies ranges from 0.2 to 68 , with a median value of 2.7 . A minimum of $19 \%$ of the total IR luminosity in the RBGS is produced in LIRGs and ultraluminous infrared galaxies with red UV colors $(\beta>0)$. Among resolved interacting systems, $32 \%$ contain one galaxy which dominates the IR emission while the companion dominates the UV emission. Only $21 \%$ of the resolved systems contain a single galaxy which dominates both wavelengths.

Key words: infrared: galaxies - ultraviolet: galaxies
\end{abstract}

\section{INTRODUCTION}

The Infrared Astronomical Satellite (IRAS) provided the first unbiased survey of the sky at mid- and far-infrared wavelengths, giving us a comprehensive census of the infrared emission properties of galaxies in the local universe. A major result of this survey was the discovery of a large population of luminous infrared galaxies (LIRGs) which emit a large majority of their bolometric luminosity in the far-infrared, and have $10^{11} L_{\odot} \leqslant$ $L_{\text {IR }}[8-1000 \mu \mathrm{m}]<10^{12} L_{\odot}$. LIRGs are a mixture of single galaxies, disk galaxy pairs, interacting systems, and advanced mergers. They exhibit enhanced star formation rates (SFRs) and a higher fraction of active galactic nuclei (AGNs) compared to less luminous and non-interacting galaxies (Sanders \& Mirabel 1996, and references therein). At the highest luminosities, ultraluminous infrared galaxies (ULIRGs; $L_{\mathrm{IR}} \geqslant 10^{12} L_{\odot}$ ) may represent an important evolutionary stage in the formation of QSOs (Sanders et al. 1988a, 1988b) and massive ellipticals (e.g., Genzel et al. 2001; Tacconi et al. 2002). Since LIRGs comprise the bulk of the cosmic infrared background and dominate the star formation activity between $0.5<z<$ 1 (Le Floc'h et al. 2005; Caputi et al. 2006), they may also play a key role in our understanding of the general evolution of galaxies and black holes (e.g., Magorrian et al. 1998).

The Great Observatories All-sky LIRG Survey (GOALS; Armus et al. 2009) contains a complete sample of low-redshift LIRGs and ULIRGs with observations across the electromagnetic spectrum. The GOALS targets are drawn from the IRAS Revised Bright Galaxy Sample (RBGS; Sanders et al. 2003), a complete sample of 629 galaxies with IRAS $60 \mu \mathrm{m}$ flux densities $S_{60}>5.24 \mathrm{Jy}$, covering the full sky above Galactic latitudes $|b|>5^{\circ}$. The 629 galaxies have a median redshift of $z=0.008$ and a maximum redshift of $z=0.088$. There are 181 LIRGs and 21 ULIRGs in the RBGS, and these galaxies define the GOALS sample. 
In LIRGs and ULIRGs, UV radiation is produced by young stars and AGN. A fraction of the UV radiation is absorbed by dust and re-radiated in the far-infrared. To understand the power sources in these galaxies, it is essential to fully characterize the energy budget by measuring both the emerging UV and the infrared flux. The relationship between the UV continuum slope and the infrared excess (the IRX- $\beta$ correlation) provides a useful parameterization of this energy budget. Charlot \& Fall (2000) showed that the IRX $-\beta$ relation is a sequence in effective optical depth for star-forming systems. However, this relation does not hold in all systems. While lower luminosity starbursts follow the correlation, ULIRGs do not (Meurer et al. 1999; Goldader et al. 2002). The GOALS sample allows us to explore the IRX- $\beta$ correlation precisely over the luminosity range where it breaks down. A detailed study of LIRGs may indicate the luminosity threshold or the time during the merger when the UV slope becomes decoupled from the IR emission. Being a flux limited sample of the nearest and most well-studied LIRGs and ULIRGs, GOALS provides an important local benchmark against which to compare the observed visual properties of high-redshift galaxies. This paper looks at global UV and IR properties. Future work will address nearby spatially resolved LIRGs.

This paper is divided into five sections. The data are discussed in Section 2. Analysis of the sample is presented in Section 3, results are discussed in Section 4, and conclusions are given in Section 5. A cosmology of $\Omega_{\Lambda}=0.72, \Omega_{m}=0.28$, with $H_{0}=70 \mathrm{~km} \mathrm{~s}^{-1} \mathrm{Mpc}^{-1}$ is adopted throughout.

\section{OBSERVATIONS AND DATA REDUCTION}

The GOALS GALEX sample consists of 135 systems observed as part of GALEX Cycle 1 program No. 13 (PI: Mazzarella), GALEX Cycle 5 program No. 38 (PI: Howell), the Nearby Galaxy Survey (NGS), and the All Sky Survey (AIS). All systems have been observed in both the FUV $\left(\lambda_{\text {eff }}=1528 \AA\right)$ and NUV $\left(\lambda_{\text {eff }}=2271 \AA\right)$. Integration times range from $\sim 100 \mathrm{~s}$ for the AIS data to $>1500 \mathrm{~s}$ for the Cycle 1, Cycle 5, and NGS data. Aside from a handful of galaxies not yet observed from the Cycle 5 program, the 135 systems described here represent all GOALS targets accessible to GALEX.

Photometry was performed on the standard GALEX pipeline data products. Since GALEX backgrounds are very low, especially for FUV images, standard photometry codes often return a background value of zero. To accurately measure the background in these images, we followed the prescription of Gil de Paz et al. (2007, hereafter GDP) using software written by those authors and made available to us. Standard IDL aperture photometry codes were used to measure the total UV fluxes inside large apertures (typically $1^{\prime}$ radius) matched to Spitzer $24 \mu \mathrm{m}$ MIPS photometry (J. Mazzarella et al. 2010, in preparation). Aperture centers were taken from Armus et al. (2009). The resultant UV GALEX photometry of the sample is presented in Table 1. In the case of widely separated pairs, only the more IR-luminous component is listed. Close pairs are treated as a single system, with the combined flux density listed in the table.

To test the accuracy of our measurements and to ensure meaningful comparisons with published data sets such as GDP, galaxies with D25 ellipses (de Vaucouleurs et al. 1991, p. 2069) were measured in D25 elliptical apertures. Little difference was found between the fluxes measured in the D25 aperture as compared to the circular aperture. The seven targets in common with the sample of GDP revealed a systematic shift in the photometric calibration between different versions of the
GALEX data reduction pipeline. To account for this, the GDP fluxes have been adjusted for purposes of comparison with the GOALS sample. The raw count rates (before background subtraction) have been multiplied by factors of 0.89 (FUV) and 1.05 (NUV). Fluxes and magnitudes were then recalculated after background subtraction.

The resolution of Spitzer allows many interacting pairs or groups to be resolved into their component galaxies in the IR. For systems with separations greater than 0.5 , the $70 \mu \mathrm{m}$ flux ratio was used to estimate the fraction of the IRAS $L_{\mathrm{IR}}$ coming from each galaxy. Similarly, the $24 \mu \mathrm{m}$ flux ratio was used for systems separated by $0.12<d<0.5$, and systems which saturated at $70 \mu \mathrm{m}$. The latter method is inaccurate for systems in which the two galaxies have different far-IR colors, such as the Arp 299 (NGC 3690/IC 0694) system (see Charmandaris et al. 2002). A total of 93 galaxies in 44 GOALS systems have been resolved in one or both GALEX FUV and NUV images. Photometry of the resolved sources is presented in Table 2.

\section{RESULTS}

\subsection{UV Luminosities and Spectral Slopes}

Although selected to be IR luminous, the GOALS sample spans a wide range of UV luminosities. The FUV flux densities range from $2.4 \times 10^{-16}$ to $2.9 \times 10^{-13} \mathrm{erg} \mathrm{s}^{-1} \mathrm{~cm}^{-2} \AA^{-1}$, with a median of $7.3 \times 10^{-15} \mathrm{erg} \mathrm{s}^{-1} \mathrm{~cm}^{-2} \AA^{-1}$ and a mean of $(1.7 \pm 0.4) \times 10^{-14} \mathrm{erg} \mathrm{s}^{-1} \mathrm{~cm}^{-2} \AA^{-1}$. The NUV flux densities range from $6.8 \times 10^{-16}$ to $2.6 \times 10^{-13} \mathrm{erg} \mathrm{s}^{-1} \mathrm{~cm}^{-2} \AA^{-1}$ with a median of $5.1 \times 10^{-15} \mathrm{erg} \mathrm{s}^{-1} \mathrm{~cm}^{-2} \AA^{-1}$ and a mean of $(1.3 \pm 0.3) \times 10^{-14} \mathrm{erg} \mathrm{s}^{-1} \mathrm{~cm}^{-2} \AA^{-1}$. The log of the FUV luminosities range from 8.30 to 10.33 , with a median and a mean of $9.45 \pm 0.04$, where the luminosities are expressed in solar units, uncorrected for reddening. The log of the NUV luminosities range from 8.30 to 10.40 , with a median of 9.57 and a mean of $9.64 \pm 0.04$. For comparison, the characteristic luminosity $L_{*}$ for the present day FUV luminosity function is $10^{9.6} L_{\odot}$ (Wyder et al. 2005). The GOALS sample is thus on average only $30 \%$ fainter than $L_{*}$ in the FUV, and the most UV-luminous LIRGs in GOALS are ultraviolet luminous galaxies (UVLGs, defined as $\log \left(L_{\mathrm{FUV}} / L_{\odot}\right)>10.3$; Heckman et al. 2005).

The infrared excess IRX is defined as the ratio of IR to FUV flux, most commonly expressed in logarithmic units. When calculating IRX we use $L_{\mathrm{IR}}$, the total IR luminosity from 8 to $1000 \mu \mathrm{m} . L_{\mathrm{IR}}$ is calculated using IRAS flux densities for integrated systems, and is allocated among resolved galaxies using MIPS flux density ratios as described above. IRAS flux densities for GOALS systems are taken from Sanders et al. (2003), MIPS flux densities for resolved galaxies within GOALS systems are taken from J. Mazzarella et al. (2010, in preparation), and luminosity distances are taken from Armus et al. (2009). IRX values range from 1.08 to 3.42 , with a median of 2.02 and a mean of $2.06 \pm 0.04$. Derived quantities are presented in Table 3 for integrated systems and in Table 4 for resolved galaxies.

The UV continuum slope $\beta(G A L E X)$ was calculated according to the definition of Kong et al. (2004):

$$
\beta(G A L E X)=\frac{\log \left(f_{\mathrm{FUV}}\right)-\log \left(f_{\mathrm{NUV}}\right)}{-0.182},
$$

where $f_{\mathrm{FUV}}$ and $f_{\mathrm{NUV}}$ are the mean flux densities per unit wavelength. Values of $\beta(G A L E X)$ range from -1.28 to 3.5, with a median of -0.16 and a mean of $0.07 \pm 0.08$. Since 
Table 1

GOALS GALEX Photometry-Integrated Systems

\begin{tabular}{|c|c|c|c|c|c|c|c|c|c|c|}
\hline $\begin{array}{l}\text { System } \\
\text { (1) }\end{array}$ & $\begin{array}{c}\text { Alternate Name } \\
\text { (2) }\end{array}$ & $\begin{array}{l}\text { R.A. (J2000) } \\
\text { (3) }\end{array}$ & $\begin{array}{c}\text { Decl. (J2000) } \\
(4)\end{array}$ & $\begin{array}{c}\log \left(L_{\mathrm{IR}}\right) \\
(5)\end{array}$ & $\begin{array}{l}t_{\mathrm{FUV}} \\
(6)\end{array}$ & $\begin{array}{c}f_{v}(\mathrm{FUV}) \\
(7) \\
\end{array}$ & $\begin{array}{c}\sigma_{\mathrm{FUV}} \\
(8)\end{array}$ & $\begin{array}{l}t_{\mathrm{NUV}} \\
(9)\end{array}$ & $\begin{array}{c}f_{v}(\mathrm{NUV}) \\
(10)\end{array}$ & $\begin{array}{c}\sigma_{\mathrm{NUV}} \\
(11) \\
\end{array}$ \\
\hline NGC 0023 & & $00^{\mathrm{h}} 09^{\mathrm{m}} 53^{\mathrm{s}} .41$ & $+25^{\circ} 55^{\prime} 25^{\prime \prime} 6$ & 11.12 & 3410 & $1.26 \mathrm{E}-14$ & $8 . \mathrm{E}-17$ & 3410 & $1.49 \mathrm{E}-14$ & 3.E-17 \\
\hline NGC 0034 & & $00^{\mathrm{h}} 11^{\mathrm{m}} 06^{\mathrm{s}} .55$ & $-12^{\circ} 06^{\prime} 26^{\prime \prime} .3$ & 11.49 & 119 & $1.53 \mathrm{E}-14$ & 4.E-16 & 119 & $1.05 \mathrm{E}-14$ & 2.E-16 \\
\hline Arp 256 & MCG-02-01-051-2 & $00^{\mathrm{h}} 18^{\mathrm{m}} 50^{\mathrm{s}} .40$ & $-10^{\circ} 22^{\prime} 08^{\prime \prime} .0$ & 11.48 & 1618 & $2.65 \mathrm{E}-14$ & 2.E-16 & 1618 & $2.50 \mathrm{E}-14$ & 6.E-17 \\
\hline ESO 350-IG038 & & $00^{\mathrm{h}} 36^{\mathrm{m}} 52^{\mathrm{s}} .50$ & $-33^{\circ} 33^{\prime} 19^{\prime \prime} .0$ & 11.28 & 3356.05 & $3.95 \mathrm{E}-14$ & 1.E-16 & 3356.05 & $2.31 \mathrm{E}-14$ & 4.E-17 \\
\hline NGC 0232 & & $00^{\mathrm{h}} 42^{\mathrm{m}} 45^{\mathrm{s}} .82$ & $-23^{\circ} 33^{\prime} 40^{\prime \prime} 9$ & 11.44 & 115 & $2.65 \mathrm{E}-15$ & 2.E-16 & 115 & $2.37 \mathrm{E}-15$ & 1.E-16 \\
\hline MCG+12-02-001 & & $00^{\mathrm{h}} 54^{\mathrm{m}} 03^{\mathrm{s}} .61$ & $+73^{\circ} 05^{\prime} 11^{\prime \prime} .8$ & 11.50 & 112 & $3.60 \mathrm{E}-16$ & 3.E-16 & 112 & $1.61 \mathrm{E}-16$ & 1.E-16 \\
\hline NGC 0317B & & $00^{\mathrm{h}} 57^{\mathrm{m}} 41^{\mathrm{s}} .67$ & $+43^{\circ} 47^{\prime} 33^{\prime \prime} .2$ & 11.19 & 110 & $2.36 \mathrm{E}-15$ & 3.E-16 & 110 & $4.29 \mathrm{E}-15$ & 1.E-16 \\
\hline IC 1623 & VV 114 & $01^{\mathrm{h}} 07^{\mathrm{m}} 47^{\mathrm{s}} .18$ & $-17^{\circ} 30^{\prime} 25^{\prime \prime} .3$ & 11.71 & 1668 & $6.40 \mathrm{E}-14$ & 2.E-16 & 1668 & $4.87 \mathrm{E}-14$ & 8.E-17 \\
\hline MCG-03-04-014 & & $01^{\mathrm{h}} 10^{\mathrm{m}} 08^{\mathrm{s}} .96$ & $-16^{\circ} 51^{\prime} 09^{\prime \prime} .8$ & 11.65 & 3241 & $3.63 \mathrm{E}-15$ & 5.E-17 & 3241 & $3.78 \mathrm{E}-15$ & 2.E-17 \\
\hline ESO 244-G012 & & $01^{\mathrm{h}} 18^{\mathrm{m}} 08^{\mathrm{s}} .10$ & $-44^{\circ} 27^{\prime} 51^{\prime \prime} .0$ & 11.38 & 117 & $8.88 \mathrm{E}-15$ & 4.E-16 & 117 & $7.59 \mathrm{E}-15$ & 1.E-16 \\
\hline CGCG 436-030 & & $01^{\mathrm{h}} 20^{\mathrm{m}} 02^{\mathrm{s}} .72$ & $+14^{\circ} 21^{\prime} 42^{\prime \prime} .9$ & 11.69 & 3178.05 & $6.42 \mathrm{E}-15$ & $6 . \mathrm{E}-17$ & 6101.5 & $8.68 \mathrm{E}-15$ & 2.E-17 \\
\hline IRAS F01364-1042 & & $01^{\mathrm{h}} 38^{\mathrm{m}} 52^{\mathrm{s}} .92$ & $-10^{\circ} 27^{\prime} 11^{\prime \prime} .4$ & 11.85 & 3284 & $2.50 \mathrm{E}-16$ & 3.E-17 & 3753 & $7.62 \mathrm{E}-16$ & 1.E-17 \\
\hline NGC 0695 & & $01^{\mathrm{h}} 51^{\mathrm{m}} 14^{\mathrm{s}} .24$ & $+22^{\circ} 34^{\prime} 56^{\prime \prime} .5$ & 11.68 & 3403 & $4.96 \mathrm{E}-15$ & $6 . \mathrm{E}-17$ & 6404 & $6.83 \mathrm{E}-15$ & 2.E-17 \\
\hline UGC 01385 & & $01^{\mathrm{h}} 54^{\mathrm{m}} 53.79$ & $+36^{\circ} 55^{\prime} 04^{\prime \prime} .6$ & 11.05 & 137 & $1.09 \mathrm{E}-14$ & 4.E-16 & 137 & $8.60 \mathrm{E}-15$ & 1.E-16 \\
\hline NGC 0877 & & $02^{\mathrm{h}} 17^{\mathrm{m}} 59^{\mathrm{s}} .64$ & $+14^{\circ} 32^{\prime} 38^{\prime \prime} .6$ & 11.10 & 1694 & $2.81 \mathrm{E}-14$ & 2.E-16 & 1694 & $2.68 \mathrm{E}-14$ & 6.E-17 \\
\hline MCG+05-06-036 & & $02^{\mathrm{h}} 23^{\mathrm{m}} 20^{\mathrm{s}} .45$ & $+32^{\circ} 11^{\prime} 34^{\prime \prime} .2$ & 11.64 & 147 & $2.29 \mathrm{E}-15$ & 2.E-16 & 147 & $3.52 \mathrm{E}-15$ & 8.E-17 \\
\hline NGC 0958 & & $02^{\mathrm{h}} 30^{\mathrm{m}} 42^{\mathrm{s}} .58$ & $-02^{\circ} 56^{\prime} 27^{\prime \prime} .2$ & 11.20 & 1696 & $1.41 \mathrm{E}-14$ & 1.E-16 & 1696 & $1.27 \mathrm{E}-14$ & 4.E-17 \\
\hline NGC 1068 & & $02^{\mathrm{h}} 42^{\mathrm{m}} 40^{\mathrm{s}} .71$ & $-00^{\circ} 00^{\prime} 47^{\prime \prime} .8$ & 11.40 & 1627 & $2.86 \mathrm{E}-13$ & 5.E-16 & 1627 & $2.64 \mathrm{E}-13$ & 2.E-16 \\
\hline UGC 02238 & & $02^{\mathrm{h}} 46^{\mathrm{m}} 17^{\mathrm{s}} .49$ & $+13^{\circ} 05^{\prime} 44^{\prime \prime} .4$ & 11 & 1316 & $1.42 \mathrm{E}-15$ & 7.E-17 & 1400 & $1.28 \mathrm{E}$ & 3.E-17 \\
\hline IRAS F02437+2122 & & $02^{\mathrm{h}} 46^{\mathrm{m}} 39^{\mathrm{s}} .15$ & $+21^{\circ} 35^{\prime} 10^{\prime \prime} 3$ & 11.16 & 120 & $\ldots$ & $\ldots$ & 120 & $3.83 \mathrm{E}-16$ & 9.E-17 \\
\hline NGC 1275 & & $03^{\mathrm{h}} 19^{\mathrm{m}} 48^{\mathrm{s}} .16$ & $+41^{\circ} 30^{\prime} 42^{\prime \prime} .1$ & 11.26 & 14563.35 & $1.78 \mathrm{E}-14$ & 5.E-17 & 17097.35 & $1.67 \mathrm{E}-14$ & 2.E-17 \\
\hline NGC 1365 & & $03^{\mathrm{h}} 33^{\mathrm{m}} 36^{\mathrm{s}} .37$ & $-36^{\circ} 08^{\prime} 25^{\prime \prime} .4$ & 11.00 & 1662.05 & $1.14 \mathrm{E}-13$ & 5.E-16 & 1662.05 & $1.05 \mathrm{E}-13$ & 2.E-16 \\
\hline IRAS F03359+1523 & & $03^{\mathrm{h}} 38^{\mathrm{m}} 46^{\mathrm{s}} .70$ & $+15^{\circ} 32^{\prime} 55^{\prime \prime} .0$ & 11.55 & 107 & $9.11 \mathrm{E}-16$ & 3.E-16 & 107 & $1.32 \mathrm{E}-15$ & 6.E-17 \\
\hline CGCG 465-012 & & $03^{\mathrm{h}} 54^{\mathrm{m}} 16^{\mathrm{s}} .08$ & $+15^{\circ} 55^{\prime} 43^{\prime \prime} 4$ & 11.20 & 133 & $3.49 \mathrm{E}-16$ & 3.E-16 & 133 & $1.68 \mathrm{E}-15$ & 1.E-16 \\
\hline IRAS $03582+6012$ & & $04^{\mathrm{h}} 02^{\mathrm{m}} 30.65$ & $+60^{\circ} 20^{\prime} 33^{\prime \prime} .4$ & 11.43 & 116 & & & 116 & $8.08 \mathrm{E}-17$ & 1.E-16 \\
\hline UGC 02982 & & $04^{\mathrm{h}} 12^{\mathrm{m}} 22^{\mathrm{s}} .45$ & $+05^{\circ} 32^{\prime} 50^{\prime \prime} 6$ & 11.20 & 113 & $4.51 \mathrm{E}-16$ & 4.E-16 & 113 & $2.46 \mathrm{E}-16$ & 1.E-16 \\
\hline ESO 550-IG025 & & $04^{\mathrm{h}} 21^{\mathrm{m}} 20^{\mathrm{s}} .00$ & $-18^{\circ} 48^{\prime} 48^{\prime \prime} .0$ & 11.51 & 129 & $2.68 \mathrm{E}-15$ & 3.E-16 & 129 & $2.80 \mathrm{E}-15$ & 1.E-16 \\
\hline IRAS $04271+3849$ & & $04^{\mathrm{h}} 30^{\mathrm{m}} 33^{\mathrm{s}} .09$ & $+38^{\circ} 55^{\prime} 47^{\prime \prime} .7$ & 11.11 & 166 & $\ldots$ & $\ldots$ & 166 & $\ldots$ & $\ldots$ \\
\hline ESO 203-IG001 & & $04^{\mathrm{h}} 46^{\mathrm{m}} 49^{\mathrm{s}} .50$ & $-48^{\circ} 33^{\prime} 32^{\prime \prime} .9$ & 11.86 & 3899 & $7.39 \mathrm{E}-16$ & 3.E-17 & 5482 & $6.81 \mathrm{E}-16$ & 1.E-17 \\
\hline MCG-05-12-006 & & $04^{\mathrm{h}} 52^{\mathrm{m}} 04^{\mathrm{s}} .96$ & $-32^{\circ} 59^{\prime} 25^{\prime \prime} 6$ & & 81 & $1.64 \mathrm{E}-15$ & 3.E-16 & 81 & $3.94 \mathrm{E}-15$ & 1.E-16 \\
\hline CGCG 468-002 & & $05^{\mathrm{h}} 08^{\mathrm{m}} 20^{\mathrm{s}} .50$ & $+17^{\circ} 21^{\prime} 58^{\prime \prime} .0$ & & 118 & E-16 & 5.E-16 & 118 & $5.07 \mathrm{E}-16$ & 1.E-16 \\
\hline IRAS $05083+2441$ & & $05^{\mathrm{h}} 11^{\mathrm{m}} 25^{\mathrm{s}} .88$ & $+24^{\circ} 45^{\prime} 18^{\prime \prime} .3$ & 11.26 & 111.05 & $2.00 \mathrm{E}-16$ & 2.E-16 & 111.05 & $3.42 \mathrm{E}-16$ & $6 . \mathrm{E}-17$ \\
\hline IRAS $05129+5128$ & & $05^{\mathrm{h}} 16^{\mathrm{m}} 56^{\mathrm{s}} 10$ & $+51^{\circ} 31^{\prime} 56^{\prime \prime} .5$ & 11.42 & 130 & 5.33E-16 & 3.E-16 & 130 & $5.90 \mathrm{E}-16$ & 1.E-16 \\
\hline IRAS F05189-2524 & & $05^{\mathrm{h}} 21^{\mathrm{m}} 01^{\mathrm{s}} 47$ & $-25^{\circ} 21^{\prime} 45^{\prime \prime} .4$ & 12.16 & 2310.05 & $1.58 \mathrm{E}-15$ & 8.E-17 & 2311.05 & $1.36 \mathrm{E}-15$ & 3.E-17 \\
\hline IRAS $05223+1908$ & & $05^{\mathrm{h}} 25^{\mathrm{m}} 16^{\mathrm{s}} 50$ & $+19^{\circ} 10^{\prime} 46^{\prime \prime} 0$ & 11.65 & 114 & $\ldots$ & $\ldots$ & 114 & $\ldots$ & 5. \\
\hline $\mathrm{MCG}+08-11-002$ & & $5^{\mathrm{h}} 40^{\mathrm{m}} 43^{\mathrm{s}} .68$ & $+49^{\circ} 41^{\prime} 35^{\prime \prime} .4$ & 11.46 & 3368 & $\ldots$ & $\ldots$ & 3368 & $8.60 \mathrm{E}-16$ & 2.E-17 \\
\hline NGC 1961 & & $05^{\mathrm{h}} 42^{\mathrm{m}} 04^{\mathrm{s}} .80$ & $+69^{\circ} 22^{\prime} 43^{\prime \prime} .3$ & 11.06 & 3932.05 & $2.86 \mathrm{E}-14$ & 1.E-16 & 3932.05 & $2.69 \mathrm{E}-14$ & 5.E-17 \\
\hline UGC 03410 & & $06^{\mathrm{h}} 14^{\mathrm{m}} 29.63$ & $+80^{\circ} 26^{\prime} 59^{\prime \prime} .6$ & 11.10 & 136 & $3.20 \mathrm{E}-15$ & 3.E-16 & 137 & $2.94 \mathrm{E}-15$ & 1.E-16 \\
\hline NGC 2146 & & $06^{\mathrm{h}} 18^{\mathrm{m}} 37^{\mathrm{s}} .71$ & $+78^{\circ} 21^{\prime} 25^{\prime \prime} .3$ & 11.12 & 1621 & $1.39 \mathrm{E}-14$ & 1.E-16 & 1621 & $1.93 \mathrm{E}-14$ & $6 . \mathrm{E}-17$ \\
\hline ESO255-IG007 & & $6^{\mathrm{h}} 27^{\mathrm{m}} 22^{\mathrm{s}} .64$ & $-47^{\circ} 10^{\prime} 48^{\prime \prime} .4$ & 11.90 & 3393 & $5.46 \mathrm{E}-15$ & 6.E-17 & 3393 & $5.21 \mathrm{E}-15$ & 2.E-17 \\
\hline NGC 2342 & & $07^{\mathrm{h}} 09^{\mathrm{m}} 18^{\mathrm{s}} .08$ & $+20^{\circ} 38^{\prime} 09^{\prime \prime} .5$ & 11.31 & 111 & $2.54 \mathrm{E}-14$ & 6.E-16 & 111 & $2.26 \mathrm{E}-14$ & 2.E-16 \\
\hline NGC 2369 & & $07^{\mathrm{h}} 16^{\mathrm{m}} 37^{\mathrm{s}} .73$ & $-62^{\circ} 20^{\prime} 37^{\prime \prime} .4$ & 11.16 & 105 & $5.13 \mathrm{E}-15$ & 4.E-16 & 105 & $4.72 \mathrm{E}-15$ & 1.E-16 \\
\hline NGC 2388 & & $07^{\mathrm{h}} 28^{\mathrm{m}} 53^{\mathrm{s}} .44$ & $+33^{\circ} 49^{\prime} 08^{\prime \prime} .7$ & 11.28 & 1700 & $2.96 \mathrm{E}-14$ & 2.E-16 & 1700 & $2.19 \mathrm{E}-14$ & 6.E-17 \\
\hline MCG+02-20-003 & & $07^{\mathrm{h}} 35^{\mathrm{m}} 43^{\mathrm{s}} .37$ & $+11^{\circ} 42^{\prime} 33^{\prime \prime} .5$ & & 117 & $1.16 \mathrm{E}-14$ & 4.E-16 & 117 & $9.14 \mathrm{E}-15$ & 2.E-16 \\
\hline NGC 2623 & & $08^{\mathrm{h}} 38^{\mathrm{m}} 24^{\mathrm{s}} .08$ & $+25^{\circ} 45^{\prime} 16^{\prime \prime} 9$ & 11.60 & 1698 & $5.44 \mathrm{E}-15$ & 8.E-17 & 1698 & $4.93 \mathrm{E}-15$ & 3.E-17 \\
\hline ESO 060-IG016 & & $8^{\mathrm{h}} 52^{\mathrm{m}} 32^{\mathrm{s}} .03$ & $-69^{\circ} 01^{\prime} 57^{\prime \prime} .3$ & 11.82 & 1580 & $1.11 \mathrm{E}-15$ & 1.E-16 & 1580 & $1.65 \mathrm{E}-15$ & 4.E-17 \\
\hline IRAS F08572+3915 & & $09^{\mathrm{h}} 00^{\mathrm{m}} 25^{\mathrm{s}} 39$ & $+39^{\circ} 03^{\prime} 54^{\prime \prime} .4$ & 12.16 & 1268.1 & $1.39 \mathrm{E}-15$ & 6.E-17 & 1268.1 & $1.94 \mathrm{E}-15$ & 3.E-17 \\
\hline IRAS 09022-3615 & & $09^{\mathrm{h}} 04^{\mathrm{m}} 12^{\mathrm{s}} \cdot 70$ & $-36^{\circ} 27^{\prime} 01^{\prime \prime} 1$ & 12.31 & 5224 & $2.38 \mathrm{E}-16$ & 6.E-17 & 5224 & $1.11 \mathrm{E}-15$ & 2.E-17 \\
\hline IRAS F09111-1007 & & $09^{\mathrm{h}} 13^{\mathrm{m}} 38^{\mathrm{s}} .80$ & $-10^{\circ} 19^{\prime} 20^{\prime \prime} .3$ & 12.06 & 2924 & $1.07 \mathrm{E}-15$ & 5.E-17 & 19292.1 & $1.84 \mathrm{E}-15$ & 8.E-18 \\
\hline UGC 04881 & & $09^{\mathrm{h}} 15^{\mathrm{m}} 55^{\mathrm{s}} .10$ & $+44^{\circ} 19^{\prime} 55^{\prime \prime} .0$ & 11.74 & 3338 & $2.52 \mathrm{E}-15$ & 4.E-17 & 3339 & $3.42 \mathrm{E}-15$ & 2.E-17 \\
\hline UGC 05101 & & $09^{\mathrm{h}} 35^{\mathrm{m}} 51^{\mathrm{s}} .65$ & $+61^{\circ} 21^{\prime} 11^{\prime \prime} .3$ & 12.01 & 1690 & $8.91 \mathrm{E}-16$ & 5.E-17 & 1690 & $1.29 \mathrm{E}-15$ & 2.E-17 \\
\hline MCG+08-18-013 & & $09^{\mathrm{h}} 36^{\mathrm{m}} 37^{\mathrm{s}} .19$ & $+48^{\circ} 28^{\prime} 27^{\prime \prime} .7$ & 11.34 & 105 & $9.76 \mathrm{E}-15$ & 4.E-16 & 105 & $6.49 \mathrm{E}-15$ & 1.E-16 \\
\hline Arp 303 & IC 0563-4 & $09^{\mathrm{h}} 46^{\mathrm{m}} 20.60$ & $+03^{\circ} 03^{\prime} 30^{\prime \prime} 0$ & 11.23 & 118 & $4.65 \mathrm{E}-15$ & 3.E-16 & 118 & $4.24 \mathrm{E}-15$ & 1.E-16 \\
\hline NGC 3110 & & $10^{\mathrm{h}} 04^{\mathrm{m}} 02^{\mathrm{s}} .11$ & $-06^{\circ} 28^{\prime} 29^{\prime \prime} 2$ & 11.37 & 80 & $1.80 \mathrm{E}-14$ & 6.E-16 & 80 & $1.67 \mathrm{E}-14$ & 2.E-16 \\
\hline ESO 374-IG032 & IRAS F10038-3338 & $10^{\mathrm{h}} 06^{\mathrm{m}} 04^{\mathrm{s}} .80$ & $-33^{\circ} 53^{\prime} 15^{\prime \prime} 0$ & 11.78 & 4930.65 & $2.28 \mathrm{E}-15$ & 5.E-17 & 14429.75 & $7.91 \mathrm{E}-15$ & 1.E-17 \\
\hline IRAS F10173+0828 & & $10^{\mathrm{h}} 20^{\mathrm{m}} 00^{\mathrm{s}} 22$ & $+08^{\circ} 13^{\prime} 34^{\prime \prime} .0$ & 11.86 & 3284.2 & $5.87 \mathrm{E}-18$ & 3.E-17 & 6789.2 & $9.63 \mathrm{E}-16$ & 1.E-17 \\
\hline NGC 3221 & & $10^{\mathrm{h}} 22^{\mathrm{m}} 19^{\mathrm{s}} .90$ & $+21^{\circ} 34^{\prime} 30^{\prime \prime} 4$ & 11.09 & 107 & $7.79 \mathrm{E}-15$ & 4.E-16 & 107 & $8.36 \mathrm{E}-15$ & 2.E-16 \\
\hline NGC 3256 & & $10^{\mathrm{h}} 27^{\mathrm{m}} 51^{\mathrm{s}} .83$ & $-43^{\circ} 54^{\prime} 13^{\prime \prime} .2$ & 11.64 & 1152 & $3.10 \mathrm{E}-14$ & 2.E-16 & 1152 & $3.72 \mathrm{E}-14$ & $9 . \mathrm{E}-17$ \\
\hline ESO 264-G036 & & $10^{\mathrm{h}} 43^{\mathrm{m}} 07^{\mathrm{s}} .67$ & $-46^{\circ} 12^{\prime} 44^{\prime \prime} 6$ & 11.32 & 109 & $7.74 \mathrm{E}-17$ & 4.E-16 & 109 & $2.70 \mathrm{E}-15$ & 1.E-16 \\
\hline IRAS F10565+2448 & & $10^{\mathrm{h}} 59^{\mathrm{m}} 18^{\mathrm{s}} .28$ & $+24^{\circ} 32^{\prime} 34^{\prime \prime} .8$ & 12.08 & 1653 & $4.00 \mathrm{E}-16$ & 4.E-17 & 1653 & $9.22 \mathrm{E}-16$ & 2.E-17 \\
\hline MCG+07-23-019 & & $11^{\mathrm{h}} 03^{\mathrm{m}} 53^{\mathrm{s}} .20$ & $+40^{\circ} 50^{\prime} 57^{\prime \prime} .0$ & 11.62 & 105 & $7.27 \mathrm{E}-15$ & 3.E-16 & 3733 & $5.19 \mathrm{E}-15$ & 2.E-17 \\
\hline CGCG 011-076 & & $11^{\mathrm{h}} 21^{\mathrm{m}} 12.26$ & $-02^{\circ} 59^{\prime} 03^{\prime \prime} .5$ & 11.43 & 106 & $5.90 \mathrm{E}-16$ & 1.E-16 & 106 & $9.41 \mathrm{E}-16$ & 6.E-17 \\
\hline IRAS F11231+1456 & IC 2810 & $11^{\mathrm{h}} 25^{\mathrm{m}} 45^{\mathrm{s}} .05$ & $+14^{\circ} 40^{\prime} 35^{\prime \prime} .9$ & 11.64 & 122 & $1.55 \mathrm{E}-15$ & 1.E-16 & 122 & $2.26 \mathrm{E}-15$ & 7.E-17 \\
\hline NGC 3690 & Arp 299 & $11^{\mathrm{h}} 28^{\mathrm{m}} 32^{\mathrm{s}} \cdot 20$ & $+58^{\circ} 33^{\prime} 44^{\prime \prime} .0$ & 11.93 & 103 & $1.19 \mathrm{E}-13$ & 1.E-15 & 103 & $8.09 \mathrm{E}-14$ & 4.E-16 \\
\hline ESO 320-G030 & & $11^{\mathrm{h}} 53^{\mathrm{m}} 11^{\mathrm{s}} .72$ & $-39^{\circ} 07^{\prime} 48^{\prime \prime} .9$ & 11.17 & 103 & $3.79 \mathrm{E}-15$ & 4.E-16 & 103 & $4.94 \mathrm{E}-15$ & 1.E-16 \\
\hline
\end{tabular}


Table 1

(Continued)

\begin{tabular}{|c|c|c|c|c|c|c|c|c|c|c|}
\hline $\begin{array}{l}\text { System } \\
\text { (1) }\end{array}$ & $\begin{array}{c}\text { Alternate Name } \\
\text { (2) }\end{array}$ & $\begin{array}{l}\text { R.A. (J2000) } \\
\text { (3) }\end{array}$ & $\begin{array}{c}\text { Decl. (J2000) } \\
\text { (4) }\end{array}$ & $\begin{array}{c}\log \left(L_{\mathrm{IR}}\right) \\
(5)\end{array}$ & $\begin{array}{l}t_{\mathrm{FUV}} \\
(6)\end{array}$ & $\begin{array}{c}f_{v}(\mathrm{FUV}) \\
(7)\end{array}$ & $\begin{array}{c}\sigma_{\mathrm{FUV}} \\
(8)\end{array}$ & $\begin{array}{c}t_{\mathrm{NUV}} \\
(9)\end{array}$ & $\begin{array}{c}f_{v}(\mathrm{NUV}) \\
(10)\end{array}$ & $\begin{array}{c}\sigma_{\mathrm{NUV}} \\
(11)\end{array}$ \\
\hline ESO 440-IG058 & & $12^{\mathrm{h}} 06^{\mathrm{m}} 51^{\mathrm{s}} .90$ & $-31^{\circ} 56^{\prime} 54^{\prime \prime} 0$ & 11.43 & 92 & $2.26 \mathrm{E}-15$ & 3.E-16 & 92 & $3.48 \mathrm{E}-15$ & 1.E-16 \\
\hline IRAS F12112+0305 & & $12^{\mathrm{h}} 13^{\mathrm{m}} 46^{\mathrm{s}} .00$ & $+02^{\circ} 48^{\prime} 38^{\prime \prime} .0$ & 12.36 & 3713 & $1.46 \mathrm{E}-15$ & 3.E-17 & 3713 & $1.14 \mathrm{E}-15$ & 2.E-17 \\
\hline ESO 267-G030 & & $12^{\mathrm{h}} 14^{\mathrm{m}} 12^{\mathrm{s}} .84$ & $-47^{\circ} 13^{\prime} 43^{\prime \prime} 2$ & 11.25 & 104 & $2.29 \mathrm{E}-15$ & 4.E-16 & 104 & $2.23 \mathrm{E}-15$ & 1.E-16 \\
\hline NGC 4922 & & $13^{\mathrm{h}} 01^{\mathrm{m}} 24^{\mathrm{s}} .90$ & $+29^{\circ} 18^{\prime} 40^{\prime \prime} 0$ & 11.38 & 1888.7 & $1.40 \mathrm{E}-15$ & 5.E-17 & 3524 & $2.19 \mathrm{E}-15$ & 2.E-17 \\
\hline CGCG 043-099 & & $13^{\mathrm{h}} 01^{\mathrm{m}} 50^{\mathrm{s}} .80$ & $+04^{\circ} 20^{\prime} 00^{\prime \prime} 0$ & 11.68 & 137 & $1.69 \mathrm{E}-15$ & 2.E-16 & 137 & $1.36 \mathrm{E}-15$ & 8.E-17 \\
\hline MCG-02-33-098-9 & & $13^{\mathrm{h}} 02^{\mathrm{m}} 19^{\mathrm{s}} .70$ & $-15^{\circ} 46^{\prime} 03^{\prime \prime} .0$ & 11.17 & 102 & $5.51 \mathrm{E}-15$ & 4.E-16 & 112 & $7.11 \mathrm{E}-15$ & 1.E-16 \\
\hline ESO 507-G070 & & $13^{\mathrm{h}} 02^{\mathrm{m}} 52^{\mathrm{s}} .35$ & $-23^{\circ} 55^{\prime} 17^{\prime \prime} .7$ & 11.56 & 1251 & $2.02 \mathrm{E}-15$ & 9.E-17 & 1251 & $1.99 \mathrm{E}-15$ & 3.E-17 \\
\hline VV 250a & & $13^{\mathrm{h}} 15^{\mathrm{m}} 35^{\mathrm{s}} .02$ & $+62^{\circ} 07^{\prime} 28^{\prime \prime} .8$ & 11.81 & 103 & $8.66 \mathrm{E}-15$ & 4.E-16 & 120 & $8.87 \mathrm{E}-15$ & 1.E-16 \\
\hline UGC 08387 & & $13^{\mathrm{h}} 20^{\mathrm{m}} 35^{\mathrm{s}} .34$ & $+34^{\circ} 08^{\prime} 22^{\prime \prime} .2$ & 11.73 & 119 & 4.13E-15 & 3.E-16 & 119 & 4.65E-15 & 1.E-16 \\
\hline NGC 5104 & & $13^{\mathrm{h}} 21^{\mathrm{m}} 23^{\mathrm{s}} .08$ & $+00^{\circ} 20^{\prime} 32^{\prime \prime} .7$ & 11.27 & 113 & $2.97 \mathrm{E}-15$ & 2.E-16 & 120 & $2.89 \mathrm{E}-15$ & 1.E-16 \\
\hline MCG-03-34-064 & & $13^{\mathrm{h}} 22^{\mathrm{m}} 24^{\mathrm{s}} .45$ & $-16^{\circ} 43^{\prime} 42^{\prime \prime} .4$ & 11.19 & 110 & $3.13 \mathrm{E}-15$ & 3.E-16 & 110 & $3.16 \mathrm{E}-15$ & 6.E-16 \\
\hline NGC 5135 & & $13^{\mathrm{h}} 25^{\mathrm{m}} 44^{\mathrm{s}} .06$ & $-29^{\circ} 50^{\prime} 01^{\prime \prime} .2$ & 11.30 & 108 & $1.80 \mathrm{E}-14$ & 6.E-16 & 108 & $1.96 \mathrm{E}-14$ & 2.E-16 \\
\hline IC 4280 & & $13^{\mathrm{h}} 32^{\mathrm{m}} 53^{\mathrm{s}} .40$ & $-24^{\circ} 12^{\prime} 25^{\prime \prime} .7$ & 11.15 & 116 & $9.92 \mathrm{E}-15$ & 4.E-16 & 116 & $1.17 \mathrm{E}-14$ & 2.E-16 \\
\hline NGC 5256 & & $13^{\mathrm{h}} 38^{\mathrm{m}} 17^{\mathrm{s}} .50$ & $+48^{\circ} 16^{\prime} 37^{\prime \prime} .0$ & 11.56 & 111 & $1.02 \mathrm{E}-14$ & 4.E-16 & 111 & $9.45 \mathrm{E}-15$ & 2.E-16 \\
\hline Arp 240 & NGC 5257-8 & $13^{\mathrm{h}} 39^{\mathrm{m}} 55^{\mathrm{s}} .20$ & $+00^{\circ} 50^{\prime} 13^{\prime \prime} .0$ & 11.62 & 129 & $3.94 \mathrm{E}-14$ & 7.E-16 & 130 & $3.08 \mathrm{E}-14$ & 2.E-16 \\
\hline UGC 08739 & & $13^{\mathrm{h}} 49^{\mathrm{m}} 13^{\mathrm{s}} .93$ & $+35^{\circ} 15^{\prime} 26^{\prime \prime} .8$ & 11.15 & 59 & $6.46 \mathrm{E}-15$ & 4.E-16 & 59 & $4.76 \mathrm{E}-15$ & 2.E-16 \\
\hline NGC 5331 & & $13^{\mathrm{h}} 52^{\mathrm{m}} 16^{\mathrm{s}} .20$ & $+02^{\circ} 06^{\prime} 16^{\prime \prime} 0$ & 11.66 & 2704 & $5.21 \mathrm{E}-15$ & 6.E-17 & 4312.5 & $5.23 \mathrm{E}-15$ & 2.E-17 \\
\hline Arp 84 & NGC 5394-5 & $13^{\mathrm{h}} 58^{\mathrm{m}} 35^{\mathrm{s}} .80$ & $+37^{\circ} 26^{\prime} 20^{\prime \prime} 0$ & 11.08 & 91 & $7.52 \mathrm{E}-15$ & 4.E-16 & 1476 & $8.88 \mathrm{E}-15$ & 4.E-17 \\
\hline CGCG 247-020 & & $14^{\mathrm{h}} 19^{\mathrm{m}} 43^{\mathrm{s}} .25$ & $+49^{\circ} 14^{\prime} 11^{\prime \prime} .7$ & 11.39 & 105 & $4.67 \mathrm{E}-16$ & 2.E-16 & 105 & $3.59 \mathrm{E}-16$ & 7.E-17 \\
\hline NGC 5653 & & $14^{\mathrm{h}} 30^{\mathrm{m}} 10^{\mathrm{s}} .42$ & $+31^{\circ} 12^{\prime} 55^{\prime \prime} .8$ & 11.13 & 92 & $1.84 \mathrm{E}-14$ & 6.E-16 & 92 & $1.85 \mathrm{E}-14$ & 2.E-16 \\
\hline IRAS F14348-1447 & & $14^{\mathrm{h}} 37^{\mathrm{m}} 38^{\mathrm{s}} 37$ & $-15^{\circ} 00^{\prime} 22^{\prime \prime} .8$ & 12.39 & 1942 & $1.32 \mathrm{E}-15$ & 7.E-17 & 3134 & $9.46 \mathrm{E}-16$ & 2.E-17 \\
\hline NGC 5734 & & $14^{\mathrm{h}} 45^{\mathrm{m}} 09^{\mathrm{s}} .05$ & $-20^{\circ} 52^{\prime} 13^{\prime \prime} .7$ & 11.15 & 128 & $1.06 \mathrm{E}-14$ & 4.E-16 & 128 & $9.61 \mathrm{E}-15$ & 2.E-16 \\
\hline VV 340a & & $14^{\mathrm{h}} 57^{\mathrm{m}} 00^{\mathrm{s}} 68$ & $+24^{\circ} 37^{\prime} 02^{\prime \prime} .7$ & 11.74 & 3042 & $5.72 \mathrm{E}-15$ & 6.E-17 & 6028 & $4.89 \mathrm{E}-15$ & 2.E-17 \\
\hline CGCG 049-057 & & $15^{\mathrm{h}} 13^{\mathrm{m}} 13^{\mathrm{s}} .10$ & $+07^{\circ} 13^{\prime} 31^{\prime \prime} .8$ & 11.35 & 88 & $7.87 \mathrm{E}-17$ & 2.E-16 & 88 & $9.31 \mathrm{E}-16$ & 9.E-17 \\
\hline VV 705 & & $15^{\mathrm{h}} 18^{\mathrm{m}} 06^{\mathrm{s}} 34$ & $+42^{\circ} 44^{\prime} 36^{\prime \prime} .7$ & 11.92 & 110 & $5.23 \mathrm{E}-15$ & 3.E-16 & 110 & $4.14 \mathrm{E}-15$ & 1.E-16 \\
\hline IRAS F15250+3608 & & $15^{\mathrm{h}} 26^{\mathrm{m}} 59^{\mathrm{s}} .40$ & $+35^{\circ} 58^{\prime} 37^{\prime \prime} .5$ & 12.08 & 168 & $2.42 \mathrm{E}-15$ & 2.E-16 & 1601 & $2.25 \mathrm{E}-15$ & 2.E-17 \\
\hline NGC 5936 & & $15^{\mathrm{h}} 30^{\mathrm{m}} 00^{\mathrm{s}} .84$ & $+12^{\circ} 59^{\prime} 21^{\prime \prime} .5$ & 11.14 & 106 & $2.38 \mathrm{E}-14$ & 6.E-16 & 106 & $2.01 \mathrm{E}-14$ & 2.E-16 \\
\hline UGC 09913 & Arp 220 & $15^{\mathrm{h}} 34^{\mathrm{m}} 57^{\mathrm{s}} .11$ & $+23^{\circ} 30^{\prime} 11^{\prime \prime} .5$ & 12.28 & 92 & $2.02 \mathrm{E}-15$ & 3.E-16 & 92 & $3.07 \mathrm{E}-15$ & 8.E-17 \\
\hline NGC 5990 & & $15^{\mathrm{h}} 46^{\mathrm{m}} 16^{\mathrm{s}} .37$ & $+02^{\circ} 24^{\prime} 55^{\prime \prime} .7$ & 11.13 & 109 & $1.08 \mathrm{E}-14$ & 4.E-16 & 109 & $9.97 \mathrm{E}-15$ & 2.E-16 \\
\hline NGC 6052 & & $16^{\mathrm{h}} 05^{\mathrm{m}} 12^{\mathrm{s}} .90$ & $+20^{\circ} 32^{\prime} 32^{\prime \prime} 0$ & 11.09 & 97 & $3.71 \mathrm{E}-14$ & 8.E-16 & 2137 & $2.70 \mathrm{E}-14$ & 5.E-17 \\
\hline NGC 6090 & & $16^{\mathrm{h}} 11^{\mathrm{m}} 40^{\mathrm{s}} .70$ & $+52^{\circ} 27^{\prime} 24^{\prime \prime} 0$ & 11.58 & 2383 & $1.38 \mathrm{E}-14$ & 1.E-16 & 14866.05 & $1.28 \mathrm{E}-14$ & 1.E-17 \\
\hline IRAS F16164-0746 & & $16^{\mathrm{h}} 19^{\mathrm{m}} 11^{\mathrm{s}} .76$ & $-07^{\circ} 54^{\prime} 04^{\prime \prime} 5$ & 11.62 & 3878 & $1.51 \mathrm{E}-15$ & 1.E-16 & 3878 & $1.26 \mathrm{E}-15$ & 3.E-17 \\
\hline CGCG 052-037 & & $16^{\mathrm{h}} 30^{\mathrm{m}} 56^{\mathrm{s}} .54$ & $+04^{\circ} 04^{\prime} 58^{\prime \prime} .4$ & 11.45 & 115 & $1.55 \mathrm{E}-15$ & 2.E-16 & 115 & $1.85 \mathrm{E}-15$ & 9.E-17 \\
\hline NGC 6240 & & $16^{\mathrm{h}} 52^{\mathrm{m}} 58^{\mathrm{s}} .89$ & $+02^{\circ} 24^{\prime} 03^{\prime \prime} .4$ & 11.93 & 108 & $6.39 \mathrm{E}-15$ & 5.E-16 & 17086.45 & $6.40 \mathrm{E}-15$ & 1.E-17 \\
\hline NGC 6286 & & $16^{\mathrm{h}} 58^{\mathrm{m}} 31^{\mathrm{s}} .38$ & $+58^{\circ} 56^{\prime} 10^{\prime \prime} .5$ & 11.37 & 122 & $7.32 \mathrm{E}-15$ & 3.E-16 & 122 & $6.46 \mathrm{E}-15$ & 1.E-16 \\
\hline IRAS F17132+5313 & & $17^{\mathrm{h}} 14^{\mathrm{m}} 20^{\mathrm{s}} .00$ & $+53^{\circ} 10^{\prime} 30^{\prime \prime} 0$ & 11.96 & 3116.75 & $1.44 \mathrm{E}-15$ & 4.E-17 & 5577.65 & $1.67 \mathrm{E}-15$ & 1.E-17 \\
\hline IRAS F17207-0014 & & $17^{\mathrm{h}} 23^{\mathrm{m}} 22^{\mathrm{s}} .16$ & $-00^{\circ} 17^{\prime} 01^{\prime \prime} .7$ & 12.46 & 4345 & $1.19 \mathrm{E}-17$ & 4.E-17 & 4345 & $8.07 \mathrm{E}-17$ & 1.E-17 \\
\hline UGC 11041 & & $17^{\mathrm{h}} 54^{\mathrm{m}} 51^{\mathrm{s}} .82$ & $+34^{\circ} 46^{\prime} 34^{\prime \prime} 4$ & 11.11 & 114 & $4.74 \mathrm{E}-15$ & 3.E-16 & 114 & $3.76 \mathrm{E}-15$ & 1.E-16 \\
\hline CGCG 141-034 & & $17^{\mathrm{h}} 56^{\mathrm{m}} 56^{\mathrm{s}} .63$ & $+24^{\circ} 01^{\prime} 01^{\prime \prime} .6$ & 11.20 & 112 & $1.01 \mathrm{E}-15$ & 3.E-16 & 112 & $5.26 \mathrm{E}-16$ & 1.E-16 \\
\hline NGC 6621 & & $18^{\mathrm{h}} 12^{\mathrm{m}} 55^{\mathrm{s}} .31$ & $+68^{\circ} 21^{\prime} 48^{\prime \prime} 4$ & 11.29 & 102 & $5.64 \mathrm{E}-15$ & 3.E-16 & 102 & $6.14 \mathrm{E}-15$ & 1.E-16 \\
\hline IC 4687 & & $18^{\mathrm{h}} 13^{\mathrm{m}} 39^{\mathrm{s}} .63$ & $-57^{\circ} 43^{\prime} 31^{\prime \prime} .3$ & 11.62 & 145 & $1.74 \mathrm{E}-14$ & 4.E-16 & 145 & $1.43 \mathrm{E}-14$ & 2.E-16 \\
\hline NGC6670AB & & $18^{\mathrm{h}} 33^{\mathrm{m}} 35^{\mathrm{s}} \cdot 60$ & $+59^{\circ} 53^{\prime} 20^{\prime \prime} 0$ & 11.65 & 2760 & $3.06 \mathrm{E}-15$ & 6.E-17 & 3531 & $3.29 \mathrm{E}-15$ & 2.E-17 \\
\hline IC 4734 & & $18^{\mathrm{h}} 38^{\mathrm{m}} 25^{\mathrm{s}} .70$ & $-57^{\circ} 29^{\prime} 25^{\prime \prime} 6$ & 11.35 & 112 & $8.06 \mathrm{E}-15$ & 4.E-16 & 112 & $6.02 \mathrm{E}-15$ & 1.E-16 \\
\hline ESO 593-IG008 & & $19^{\mathrm{h}} 14^{\mathrm{m}} 30^{\mathrm{s}} .90$ & $-21^{\circ} 19^{\prime} 07^{\prime \prime} 0$ & 11.93 & 82 & $2.51 \mathrm{E}-15$ & 5.E-16 & 4422 & $5.54 \mathrm{E}-15$ & 3.E-17 \\
\hline IRAS F19297-0406 & & $19^{\mathrm{h}} 32^{\mathrm{m}} 21^{\mathrm{s}} .25$ & $-03^{\circ} 59^{\prime} 56^{\prime \prime} .3$ & 12.45 & 98.75 & $1.72 \mathrm{E}-17$ & 7.E-18 & 3802 & $2.06 \mathrm{E}-17$ & 4.E-18 \\
\hline ESO 339-G011 & & $19^{\mathrm{h}} 57^{\mathrm{m}} 37^{\mathrm{s}} .54$ & $-37^{\circ} 56^{\prime} 08^{\prime \prime} .4$ & 11.20 & 113 & $2.08 \mathrm{E}-15$ & 3.E-16 & 113 & $2.86 \mathrm{E}-15$ & 1.E-16 \\
\hline NGC 6907 & & $20^{\mathrm{h}} 25^{\mathrm{m}} 06^{\mathrm{s}} .65$ & $-24^{\circ} 48^{\prime} 33^{\prime \prime} .5$ & 11.11 & 1686 & $8.18 \mathrm{E}-14$ & 3.E-16 & 2175 & $6.30 \mathrm{E}-14$ & 8.E-17 \\
\hline NGC 6926 & & $20^{\mathrm{h}} 33^{\mathrm{m}} 06^{\mathrm{s}} .11$ & $-02^{\circ} 01^{\prime} 39^{\prime \prime} .0$ & 11.32 & 119 & $1.41 \mathrm{E}-14$ & 5.E-16 & 119 & $1.02 \mathrm{E}-14$ & 2.E-16 \\
\hline CGCG 448-020 & II Zw 096 & $20^{\mathrm{h}} 57^{\mathrm{m}} 23^{\mathrm{s}} .29$ & $+17^{\circ} 07^{\prime} 34^{\prime \prime} .3$ & 11.94 & 66 & $1.64 \mathrm{E}-14$ & 6.E-16 & 66 & $1.02 \mathrm{E}-14$ & 2.E-16 \\
\hline ESO 286-IG019 & & $20^{\mathrm{h}} 58^{\mathrm{m}} 26^{\mathrm{s}} .79$ & $-42^{\circ} 39^{\prime} 00^{\prime \prime} 3$ & 12.06 & 2974 & $5.51 \mathrm{E}-15$ & $6 . E-17$ & 8100.05 & $5.06 \mathrm{E}-15$ & 1.E-17 \\
\hline ESO 286-G035 & & $21^{\mathrm{h}} 04^{\mathrm{m}} 11^{\mathrm{s}} .18$ & $-43^{\circ} 35^{\prime} 33^{\prime \prime} .0$ & 11.20 & 105 & $4.35 \mathrm{E}-15$ & 3.E-16 & 105 & $4.72 \mathrm{E}-15$ & 1.E-16 \\
\hline ESO 343-IG013 & & $21^{\mathrm{h}} 36^{\mathrm{m}} 11^{\mathrm{s}} .00$ & $-38^{\circ} 32^{\prime} 37^{\prime \prime} .0$ & 11.14 & 81 & $3.95 \mathrm{E}-15$ & 3.E-16 & 81 & $4.24 \mathrm{E}-15$ & 1.E-16 \\
\hline NGC 7130 & & $21^{\mathrm{h}} 48^{\mathrm{m}} 19^{\mathrm{s}} .50$ & $-34^{\circ} 57^{\prime} 04^{\prime \prime} 7$ & 11.42 & 1692 & $2.11 \mathrm{E}-14$ & 1.E-16 & 1692 & $2.42 \mathrm{E}-14$ & 6.E-17 \\
\hline IC 5179 & & $22^{\mathrm{h}} 16^{\mathrm{m}} 09^{\mathrm{s}} .10$ & $-36^{\circ} 50^{\prime} 37^{\prime \prime} 4$ & 11.24 & 86 & $3.36 \mathrm{E}-14$ & 8.E-16 & 86 & $3.17 \mathrm{E}-14$ & 3.E-16 \\
\hline ESO 602-G025 & & $22^{\mathrm{h}} 31^{\mathrm{m}} 25^{\mathrm{s}} .48$ & $-19^{\circ} 02^{\prime} 04^{\prime \prime} 1$ & 11.34 & 62 & $3.63 \mathrm{E}-15$ & 4.E-16 & 62 & $3.38 \mathrm{E}-15$ & 2.E-16 \\
\hline UGC 12150 & & $22^{\mathrm{h}} 41^{\mathrm{m}} 12^{\mathrm{s}} .26$ & $+34^{\circ} 14^{\prime} 57^{\prime \prime} .0$ & 11.35 & 167 & $9.13 \mathrm{E}-17$ & 2.E-16 & 167 & 8.54E-16 & 8.E-17 \\
\hline IRAS F22491-1808 & & $22^{\mathrm{h}} 51^{\mathrm{m}} 49^{\mathrm{s}} .26$ & $-17^{\circ} 52^{\prime} 23^{\prime \prime} .5$ & 12.20 & 1696 & $1.89 \mathrm{E}-15$ & 6.E-17 & 1696 & $2.04 \mathrm{E}-15$ & 3.E-17 \\
\hline NGC 7469 & & $23^{\mathrm{h}} 03^{\mathrm{m}} 15^{\mathrm{s}} .62$ & $+08^{\circ} 52^{\prime} 26^{\prime \prime} .4$ & 11.65 & 3768 & $4.50 \mathrm{E}-14$ & 1.E-16 & 3768 & $3.29 \mathrm{E}-14$ & 4.E-17 \\
\hline CGCG 453-062 & & $23^{\mathrm{h}} 04^{\mathrm{m}} 56^{\mathrm{s}} .53$ & $+19^{\circ} 33^{\prime} 08^{\prime \prime} 0$ & 11.38 & 113 & $7.51 \mathrm{E}-16$ & 2.E-16 & 113 & $1.29 \mathrm{E}-15$ & 9.E-17 \\
\hline ESO 148-IG002 & & $23^{\mathrm{h}} 15^{\mathrm{m}} 46^{\mathrm{s}} .78$ & $-59^{\circ} 03^{\prime} 15^{\prime \prime} 6$ & 12.06 & 3009.05 & $5.88 \mathrm{E}-15$ & 6.E-17 & 5350.05 & $5.22 \mathrm{E}-15$ & 2.E-17 \\
\hline IC 5298 & & $23^{\mathrm{h}} 16^{\mathrm{m}} 00^{\mathrm{s}} .70$ & $+25^{\circ} 33^{\prime} 24^{\prime \prime} 1$ & 11.60 & 113 & $2.21 \mathrm{E}-15$ & 3.E-16 & 113 & $1.76 \mathrm{E}-15$ & 1.E-16 \\
\hline NGC 7552 & & $23^{\mathrm{h}} 16^{\mathrm{m}} 10^{\mathrm{s}} .77$ & $-42^{\circ} 35^{\prime} 05^{\prime \prime} 4$ & 11.11 & 4825 & 7.33E-14 & 1.E-16 & 7560.95 & $8.68 \mathrm{E}-14$ & 5.E-17 \\
\hline NGC 7592 & & $23^{\mathrm{h}} 18^{\mathrm{m}} 22^{\mathrm{s}} .54$ & $-04^{\circ} 24^{\prime} 58^{\prime \prime} .5$ & 11.40 & 107 & $2.32 \mathrm{E}-14$ & 6.E-16 & 107 & $1.38 \mathrm{E}-14$ & 2.E-16 \\
\hline ESO 077-IG014 & & $23^{\mathrm{h}} 21^{\mathrm{m}} 04^{\mathrm{s}} .30$ & $-69^{\circ} 12^{\prime} 54^{\prime \prime} 0$ & 11.76 & 2060 & $5.34 \mathrm{E}-16$ & 5.E-17 & 6625.05 & $1.03 \mathrm{E}-15$ & 1.E-17 \\
\hline NGC 7674 & & $23^{\mathrm{h}} 27^{\mathrm{m}} 56^{\mathrm{s}} .72$ & $+08^{\circ} 46^{\prime} 44^{\prime \prime} .5$ & 11.56 & 1666 & $1.42 \mathrm{E}-14$ & 1.E-16 & 1666 & $1.16 \mathrm{E}-14$ & 4.E-17 \\
\hline
\end{tabular}


Table 1

(Continued)

\begin{tabular}{|c|c|c|c|c|c|c|c|c|c|c|}
\hline $\begin{array}{l}\text { System } \\
\text { (1) }\end{array}$ & $\begin{array}{l}\text { Alternate Name } \\
\text { (2) }\end{array}$ & $\begin{array}{l}\text { R.A. (J2000) } \\
\text { (3) }\end{array}$ & $\begin{array}{c}\text { Decl. (J2000) } \\
\text { (4) }\end{array}$ & $\begin{array}{c}\log \left(L_{\mathrm{IR}}\right) \\
(5)\end{array}$ & $\begin{array}{c}t_{\mathrm{FUV}} \\
(6)\end{array}$ & $\begin{array}{c}f_{v}(\mathrm{FUV}) \\
(7)\end{array}$ & $\begin{array}{c}\sigma_{\mathrm{FUV}} \\
(8)\end{array}$ & $\begin{array}{l}t_{\mathrm{NUV}} \\
(9)\end{array}$ & $\begin{array}{c}f_{v}(\mathrm{NUV}) \\
(10)\end{array}$ & $\begin{array}{c}\sigma_{\mathrm{NUV}} \\
(11)\end{array}$ \\
\hline IRAS F23365+3604 & & $23^{\mathrm{h}} 39^{\mathrm{m}} 01^{\mathrm{s}} .27$ & $+36^{\circ} 21^{\prime} 08^{\prime \prime} .7$ & 12.20 & 3876 & $6.02 \mathrm{E}-16$ & 5.E-17 & 3876 & $2.62 \mathrm{E}-15$ & 2.E-17 \\
\hline MCG-01-60-022 & & $23^{\mathrm{h}} 41^{\mathrm{m}} 54^{\mathrm{s}} .10$ & $-03^{\circ} 38^{\prime} 29^{\prime \prime} .0$ & 11.27 & 1489 & $7.28 \mathrm{E}-15$ & 9.E-17 & 1489 & $5.50 \mathrm{E}-15$ & 3.E-17 \\
\hline Arp 86 & NGC 7752-3 & $23^{\mathrm{h}} 47^{\mathrm{m}} 01^{\mathrm{s}} .63$ & $+29^{\circ} 28^{\prime} 17^{\prime \prime} .2$ & 11.07 & 1687.7 & $1.02 \mathrm{E}-14$ & 1.E-16 & 3246 & $9.15 \mathrm{E}-15$ & 3.E-17 \\
\hline NGC 7771 & & $23^{\mathrm{h}} 51^{\mathrm{m}} 24^{\mathrm{s}} 88$ & $+20^{\circ} 06^{\prime} 42^{\prime \prime} 6$ & 11.40 & 8579 & 3.39E-14 & 8.E-17 & 16457 & $2.35 \mathrm{E}-14$ & 2.E-17 \\
\hline MRK0331 & & $23^{\mathrm{h}} 51^{\mathrm{m}} 26^{\mathrm{s}} .80$ & $+20^{\circ} 35^{\prime} 09^{\prime \prime} .9$ & 11.50 & 1129 & $2.91 \mathrm{E}-15$ & 1.E-16 & 1129 & 3.07E-15 & 4.E-17 \\
\hline
\end{tabular}

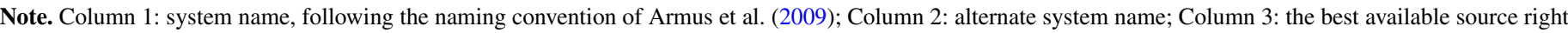

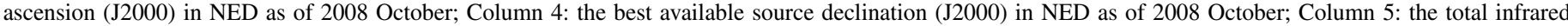

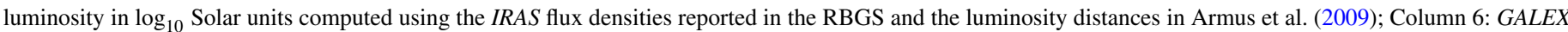

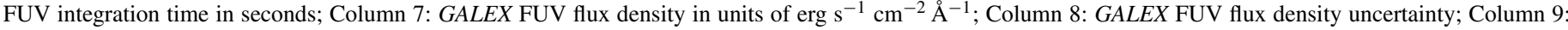

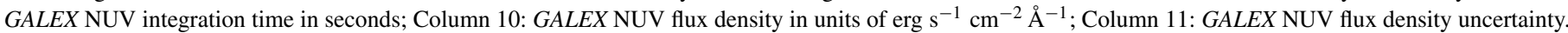

the GALEX filters have different effective wavelengths than previous instruments such as IUE or STIS, the normalization of $\beta(G A L E X)$ is different from previous work (e.g., Meurer et al. 1999; Goldader et al. 2002; see the Appendix for a more detailed discussion and a direct conversion between $\beta(G A L E X)$ and $\beta(I U E)$ ). Of the 135 observed systems, 112 have good quality data $\left(\sigma_{\beta(\text { GALEX })}<0.5\right)$ and are used in the subsequent analysis. Eleven galaxies in the Meurer et al. (1999) sample are included in GDP. These systems allow us to recreate the linear portion of the IRX- $\beta(G A L E X)$ relation for (sub-LIRG) starburst galaxies, hereafter referred to as the starburst relation. The 11 Meurer et al. (1999) systems in GDP span a range of $-1<\beta(G A L E X)<0.5$, and extrapolations beyond that range are not necessarily correct.

The IRX- $\beta(G A L E X)$ plot is shown in Figure 1. As Goldader et al. (2002) discovered, IR-luminous systems tend to lie above the starburst relation. Similarly, as seen in Kong et al. (2004), Cortese et al. (2006), and GDP, the starburst relation forms an upper envelope for normal galaxies on this plot. Within the valid range for the starburst relation, $15 \%$ of LIRGs fall below the relation. In addition, 12 LIRGs with very red UV colors $(\beta>1)$ have high IRX values (2.2-3.3) but lie far below a linear extrapolation to the starburst relation. The fit to the late-type galaxy sample of Cortese et al. (2006) provides a particularly clean separation between (U)LIRGs and sub-LIRGs in Figure 1. The shallower slope better matches the distributions of GOALS subpopulations $\left(L_{\mathrm{IR}}<10^{11.4}, 10^{11.4}<L_{\mathrm{IR}}<10^{11.8}\right.$, and $L_{\mathrm{IR}}>$ $10^{11.8}$ ), with the more luminous subpopulations having larger separations in IRX from the Cortese relation. The best fit slope for the GOALS data is IRX $=(0.46 \pm 0.06) \beta+(2.1 \pm 0.1)$, shallower than the Cortese relation by 0.24 but offset to higher IRX by 0.8 at $\beta=0$.

\subsection{Star Formation Rates}

The combination of IRAS $L_{\mathrm{IR}}$ and GALEX FUV measurements provides an estimate of the total (obscured plus unobscured) SFR (Kennicutt 1998; Dale et al. 2007). The contribution of the FUV to the measured SFR ranges from $0.2 \%$ to $17.9 \%$, with a median of $2.8 \%$ and a mean of $4.0 \% \pm 0.4 \%$. A histogram showing the ratio of UV-derived SFR to that derived from the combination of UV and IR luminosity is shown in Figure 2(a). Calculations relating to SFR do not include galaxies with IRAC colors that are consistent with the presence of a strong AGN (Stern et al. 2005). The distribution of the FUV contribution to SFR is consistent with previous work (Surace \& Sanders 2000; Surace et al. 2000). The FUV contribution to SFR is small for LIRGs and ULIRGs, and decreases as $L_{\mathrm{IR}}$ in-

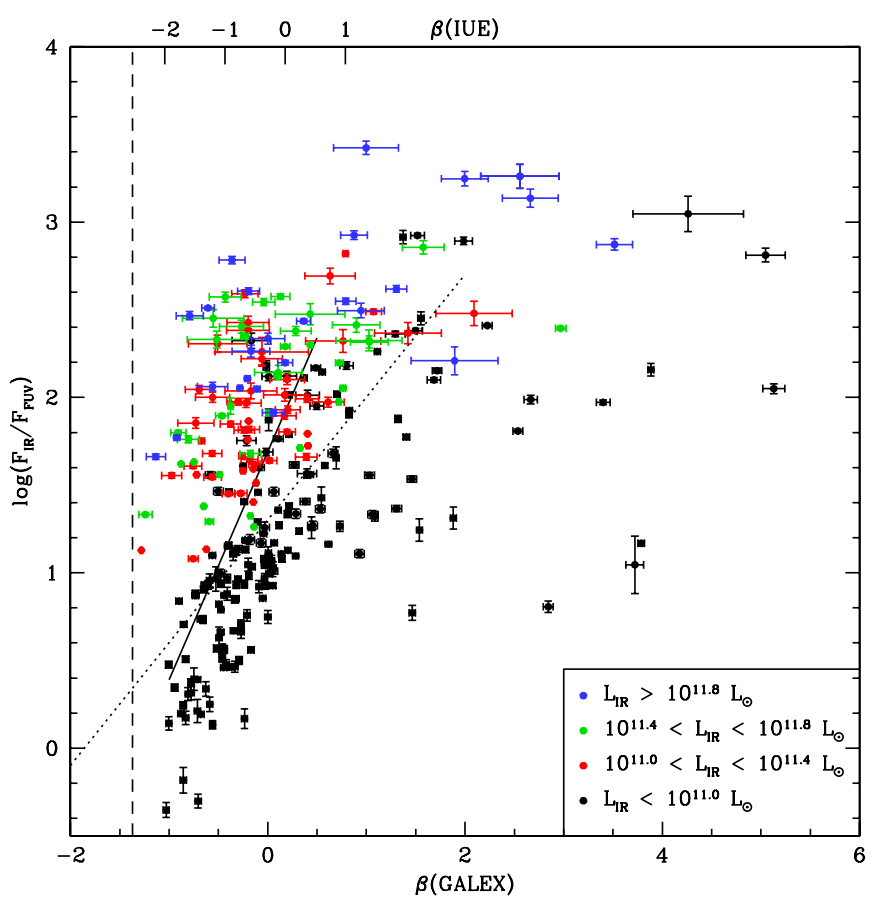

Figure 1. IR excess, $F_{\mathrm{IR}} / \mathrm{F}_{\mathrm{FUV}}$, plotted against the UV continuum slope, $\beta(G A L E X)$. Black points (from GDP) have $L_{\mathrm{IR}}<10^{11} L_{\odot}$, red points have $10^{11} L_{\odot}<L_{\mathrm{IR}}<10^{11.4} L_{\odot}$, green points have $10^{11.4} L_{\odot}<L_{\mathrm{IR}}<10^{11.8} L_{\odot}$, and blue points have $L_{\mathrm{IR}}>10^{11.8} L_{\odot}$. The solid line is a fit to the starburst galaxies of Meurer et al. (1999) which were included in the GDP sample. The dotted line is the fit to the late-type galaxy sample of Cortese et al. (2006). The vertical dashed line is the UV color of a Starburst99 (Leitherer et al. 1999) model of a $10^{8} \mathrm{yr}$ old starburst population with solar metallicity and a Salpeter IMF (Salpeter 1955). The range of $\beta(G A L E X)$ in the IUE system of Meurer et al. (1999) is shown at top. Low and medium luminosity LIRGs (red and green points) fill parameter space between normal galaxies and high luminosity LIRGs and ULIRGs (blue points).

creases (Figure 2(b)). The Spearman rank correlation coefficient $r_{s}=-0.47$, with significance $3.6 \times 10^{-6}$ indicating a significant correlation, although the relation is clearly nonlinear. Galaxies with larger infrared luminosity have a higher fraction of their measured star formation emerging in the far-infrared, with a corresponding lower fraction emerging in the far-ultraviolet. As a function of IR luminosity, the median (mean) contribution of the FUV to the measured SFR is 3.3\% (4.6\%) for systems with $L_{\mathrm{IR}}<10^{11.8}$, and drops to $1.9 \%$ (2.0\%) for systems with $L_{\mathrm{IR}}>10^{11.8}$

IRAC $3.6 \mu \mathrm{m}$ and Two Micron All Sky Survey (2MASS) $K$-band photometry were used to estimate the stellar mass of each galaxy (Lacey et al. 2008). The mass estimates 
Table 2

GALEX Photometry—Resolved Components

\begin{tabular}{|c|c|c|c|c|c|c|c|c|}
\hline $\begin{array}{c}\text { System Name } \\
\text { (1) }\end{array}$ & $\begin{array}{c}\text { Galaxy Name } \\
(2)\end{array}$ & $\begin{array}{c}\text { R.A. (J2000) } \\
\text { (3) }\end{array}$ & $\begin{array}{c}\text { Decl. (J2000) } \\
(4)\end{array}$ & $\begin{array}{c}\log \left(L_{\mathrm{IR}}\right) \\
(5)\end{array}$ & $\begin{array}{c}f_{v}(\mathrm{FUV}) \\
(6) \\
\end{array}$ & $\begin{array}{c}\sigma_{\mathrm{FUV}} \\
(7)\end{array}$ & $\begin{array}{c}f_{v}(\mathrm{NUV}) \\
(8) \\
\end{array}$ & $\begin{array}{c}\sigma_{\mathrm{NUV}} \\
(9)\end{array}$ \\
\hline GOALS J001108.5-120351 & NGC 0034 & $00^{\mathrm{h}} 11^{\mathrm{m}} 06^{\mathrm{s}} .56$ & $-12^{\circ} 06^{\prime} 28^{\prime \prime} .2$ & 11.34 & $1.53 \mathrm{E}-14$ & 4.E-16 & $1.05 \mathrm{E}-14$ & $6 . \mathrm{E}-16$ \\
\hline GOALS J001108.5-120351 & NGC 0035 & $00^{\mathrm{h}} 11^{\mathrm{m}} 10^{\mathrm{s}} .51$ & $-12^{\circ} 01^{\prime} 14^{\prime \prime} .9$ & 10.57 & $6.92 \mathrm{E}-15$ & 3.E-16 & $8.63 \mathrm{E}-15$ & $6 . \mathrm{E}-16$ \\
\hline MCG-02-01-051-2 & MCG-02-01-052 & $00^{\mathrm{h}} 18^{\mathrm{m}} 49^{\mathrm{s}} .85$ & $-10^{\circ} 21^{\prime} 34^{\prime \prime} 0$ & 10.36 & $1.24 \mathrm{E}-14$ & 1.E-16 & $1.05 \mathrm{E}-14$ & 1.E-16 \\
\hline MCG-02-01-051-2 & MCG-02-01-051 & $00^{\mathrm{h}} 18^{\mathrm{m}} 50^{\mathrm{s}} .90$ & $-10^{\circ} 22^{\prime} 36^{\prime \prime} .7$ & 11.45 & $1.42 \mathrm{E}-14$ & 1.E-16 & $1.08 \mathrm{E}-14$ & 1.E-16 \\
\hline NGC 0232 & NGC 0232 & $00^{\mathrm{h}} 42^{\mathrm{m}} 45^{\mathrm{s}} .83$ & $-23^{\circ} 33^{\prime} 41^{\prime \prime} .0$ & 11.37 & $2.65 \mathrm{E}-15$ & 2.E-16 & $2.37 \mathrm{E}-15$ & 5.E-16 \\
\hline NGC 0232 & NGC 0235A & $00^{\mathrm{h}} 42^{\mathrm{m}} 52^{\mathrm{s}} .82$ & $-23^{\circ} 32^{\prime} 27^{\prime \prime} .8$ & 10.86 & $1.28 \mathrm{E}-15$ & 2.E-16 & $1.19 \mathrm{E}-15$ & 5.E-16 \\
\hline NGC 0317 & NGC 0317A & $00^{\mathrm{h}} 57^{\mathrm{m}} 39^{\mathrm{s}} .04$ & $+43^{\circ} 48^{\prime} 03^{\prime \prime} .1$ & 8.96 & $2.48 \mathrm{E}-16$ & 3.E-17 & $1.37 \mathrm{E}-15$ & 4.E-17 \\
\hline NGC 0317 & NGC 0317B & $00^{\mathrm{h}} 57^{\mathrm{m}} 40^{\mathrm{s}} .41$ & $+43^{\circ} 47^{\prime} 32^{\prime \prime} .5$ & 11.19 & $2.12 \mathrm{E}-15$ & 3.E-16 & $2.91 \mathrm{E}-15$ & 9.E-17 \\
\hline IC 1623 & IC $1623 \mathrm{~A}$ & $01^{\mathrm{h}} 07^{\mathrm{m}} 46^{\mathrm{s}} .75$ & $-17^{\circ} 30^{\prime} 26^{\prime \prime} .2$ & 11.08 & $6.16 \mathrm{E}-14$ & 2.E-16 & $4.57 \mathrm{E}-14$ & 7.E-17 \\
\hline IC 1623 & IC 1623B & $01^{\mathrm{h}} 07^{\mathrm{m}} 47^{\mathrm{s}} .54$ & $-17^{\circ} 30^{\prime} 25^{\prime \prime} .1$ & 11.59 & $2.31 \mathrm{E}-15$ & 8.E-18 & $3.03 \mathrm{E}-15$ & 5.E-18 \\
\hline ESO 244-G012 & ESO 244-G012 NED01 & $01^{\mathrm{h}} 18^{\mathrm{m}} 08.23$ & $-44^{\circ} 28^{\prime} 00^{\prime \prime} 4$ & 9.49 & 7.27E-15 & 3.E-16 & $5.61 \mathrm{E}-15$ & 1.E-16 \\
\hline ESO 244-G012 & ESO 244-G012 NED02 & $01^{\mathrm{h}} 18^{\mathrm{m}} 08^{\mathrm{s}} .31$ & $-44^{\circ} 27^{\prime} 43^{\prime \prime} .4$ & 11.37 & $1.61 \mathrm{E}-15$ & 6.E-17 & $1.98 \mathrm{E}-15$ & 3.E-17 \\
\hline GOALS J015457.8+365508 & UGC 01385 & $01^{\mathrm{h}} 54^{\mathrm{m}} 53.82$ & $+36^{\circ} 55^{\prime} 04^{\prime \prime} .3$ & 11.00 & $1.92 \mathrm{E}-15$ & 2.E-16 & $1.71 \mathrm{E}-15$ & 3.E-16 \\
\hline GOALS J015457.8+365508 & KUG $0152+366$ & $01^{\mathrm{h}} 55^{\mathrm{m}} 01^{\mathrm{s}} .75$ & $+36^{\circ} 55^{\prime} 11^{\prime \prime} .6$ & 10.05 & $8.94 \mathrm{E}-15$ & 3.E-16 & $6.89 \mathrm{E}-15$ & 4.E-16 \\
\hline GOALS J021756.5+143158 & NGC 0876 & $02^{\mathrm{h}} 17^{\mathrm{m}} 53^{\mathrm{s}} .26$ & $+14^{\circ} 31^{\prime} 18^{\prime \prime} .4$ & 10.51 & $2.81 \mathrm{E}-14$ & 2.E-16 & $2.68 \mathrm{E}-14$ & 2.E-16 \\
\hline GOALS J021756.5+143158 & NGC 0877 & $02^{\mathrm{h}} 17^{\mathrm{m}} 59.68$ & $+14^{\circ} 32^{\prime} 38^{\prime \prime} .2$ & 11.07 & $1.27 \mathrm{E}-15$ & 6.E-17 & $1.31 \mathrm{E}-15$ & 2.E-16 \\
\hline MRK1034 & MCG+05-06-035 & $02^{\mathrm{h}} 23^{\mathrm{m}} 18^{\mathrm{s}} .97$ & $+32^{\circ} 11^{\prime} 18^{\prime \prime} .5$ & 11.00 & $1.61 \mathrm{E}-15$ & 1.E-16 & $2.53 \mathrm{E}-15$ & 6.E-17 \\
\hline MRK1034 & MCG+05-06-036 & $02^{\mathrm{h}} 23^{\mathrm{m}} 21^{\mathrm{s}} .99$ & $+32^{\circ} 11^{\prime} 48^{\prime \prime} .8$ & 11.53 & $6.80 \mathrm{E}-16$ & 5.E-17 & $9.82 \mathrm{E}-16$ & 2.E-17 \\
\hline KPG 095 & UGC 02894 & $03^{\mathrm{h}} 54^{\mathrm{m}} 07^{\mathrm{s}} .67$ & $+15^{\circ} 59^{\prime} 24^{\prime \prime} 3$ & 10.81 & $1.56 \mathrm{E}-15$ & 3.E-16 & $1.36 \mathrm{E}-15$ & 7.E-16 \\
\hline KPG 095 & CGCG 465-012 & $03^{\mathrm{h}} 54^{\mathrm{m}} 15^{\mathrm{s}} .95$ & $+15^{\circ} 55^{\prime} 43^{\prime \prime} .4$ & 11.16 & $3.49 \mathrm{E}-16$ & 3.E-16 & $1.68 \mathrm{E}-15$ & 7.E-16 \\
\hline ESO 550-IG025 & ESO 550-IG025 NED01 & $04^{\mathrm{h}} 21^{\mathrm{m}} 20^{\mathrm{s}} .02$ & $-18^{\circ} 48^{\prime} 39^{\prime \prime} 6$ & 11.27 & $2.18 \mathrm{E}-15$ & 2.E-16 & $2.17 \mathrm{E}-15$ & 8.E-17 \\
\hline ESO 550-IG025 & ESO 550-IG025 NED02 & $04^{\mathrm{h}} 21^{\mathrm{m}} 20.08$ & $-18^{\circ} 48^{\prime} 57^{\prime \prime} .4$ & 11.13 & $5.00 \mathrm{E}-16$ & 5.E-17 & $6.30 \mathrm{E}-16$ & 2.E-17 \\
\hline CGCG 468-002 & CGCG 468-002 NED01 & $05^{\mathrm{h}} 08^{\mathrm{m}} 19.71$ & $+17^{\circ} 21^{\prime} 47^{\prime \prime} .8$ & 10.74 & $4.20 \mathrm{E}-17$ & 3.E-17 & $2.65 \mathrm{E}-16$ & 7.E-17 \\
\hline CGCG 468-002 & CGCG 468-002 NED02 & $05^{\mathrm{h}} 08^{\mathrm{m}} 21^{\mathrm{s}} .21$ & $+17^{\circ} 22^{\prime} 08^{\prime \prime} .0$ & 11.05 & $7.09 \mathrm{E}-16$ & 4.E-16 & $2.42 \mathrm{E}-16$ & 6.E-17 \\
\hline GOALS J051127.4+244539 & IRAS $05083+2441$ & $05^{\mathrm{h}} 11^{\mathrm{m}} 25^{\mathrm{s}} .88$ & $+24^{\circ} 45^{\prime} 18^{\prime \prime} .2$ & 11.24 & $1.99 \mathrm{E}-16$ & 2.E-16 & $3.15 \mathrm{E}-16$ & 4.E-16 \\
\hline GOALS J051127.4+244539 & 2MASX J05112888+2445593 & $05^{\mathrm{h}} 11^{\mathrm{m}} 29.05$ & $+24^{\circ} 46^{\prime} 04^{\prime \prime} 0$ & 10.06 & $1.02 \mathrm{E}-18$ & 2.E-16 & $2.66 \mathrm{E}-17$ & 4.E-16 \\
\hline KPG 108 & UGC 03405 & $06^{\mathrm{h}} 13^{\mathrm{m}} 57^{\mathrm{s}} .90$ & $+80^{\circ} 28^{\prime} 34^{\prime \prime} .7$ & 10.54 & $3.20 \mathrm{E}-15$ & 3.E-16 & $2.94 \mathrm{E}-15$ & 5.E-16 \\
\hline KPG 108 & UGC 03410 & $06^{\mathrm{h}} 14^{\mathrm{m}} 29^{\mathrm{s}} .61$ & $+80^{\circ} 26^{\prime} 59^{\prime \prime} 6$ & 11.02 & $1.04 \mathrm{E}-15$ & 2.E-16 & $1.12 \mathrm{E}-15$ & 5.E-16 \\
\hline KPG 125 & NGC 2341 & $07^{\mathrm{h}} 09^{\mathrm{m}} 12^{\mathrm{s}} .01$ & $+20^{\circ} 36^{\prime} 11^{\prime \prime} .2$ & 11.11 & $4.98 \mathrm{E}-15$ & 4.E-16 & $6.98 \mathrm{E}-15$ & 8.E-16 \\
\hline KPG 125 & NGC 2342 & $07^{\mathrm{h}} 09^{\mathrm{m}} 18^{\mathrm{s}} .07$ & $+20^{\circ} 38^{\prime} 10^{\prime \prime} .2$ & 11.20 & $2.54 \mathrm{E}-14$ & 6.E-16 & $2.26 \mathrm{E}-14$ & 9.E-16 \\
\hline WBL 142 & NGC 2385 & $07^{\mathrm{h}} 28^{\mathrm{m}} 28^{\mathrm{s}} .17$ & $+33^{\circ} 50^{\prime} 16^{\prime \prime} .9$ & 9.59 & $3.22 \mathrm{E}-16$ & 6.E-17 & $1.86 \mathrm{E}-15$ & 2.E-16 \\
\hline WBL 142 & NGC 2388 & $07^{\mathrm{h}} 28^{\mathrm{m}} 53^{\mathrm{s}} .44$ & $+33^{\circ} 49^{\prime} 07^{\prime \prime} .8$ & 11.26 & $2.96 \mathrm{E}-14$ & 2.E-16 & $2.19 \mathrm{E}-14$ & 2.E-16 \\
\hline WBL 142 & NGC 2389 & $07^{\mathrm{h}} 29^{\mathrm{m}} 04^{\mathrm{s}} .59$ & $+33^{\circ} 51^{\prime} 38^{\prime \prime} 0$ & 10.65 & $7.20 \mathrm{E}-17$ & 6.E-17 & $3.62 \mathrm{E}-16$ & 2.E-16 \\
\hline GOALS J073542.4+113938 & NGC 2416 & $07^{\mathrm{h}} 35^{\mathrm{m}} 41^{\mathrm{s}} .53$ & $+11^{\circ} 36^{\prime} 42^{\prime \prime} .1$ & $\ldots$ & $1.16 \mathrm{E}-14$ & 4.E-16 & $9.14 \mathrm{E}-15$ & 7.E-16 \\
\hline GOALS J073542.4+113938 & MCG+02-20-003 & $07^{\mathrm{h}} 35^{\mathrm{m}} 43^{\mathrm{s}} .44$ & $+11^{\circ} 42^{\prime} 34^{\prime \prime} .8$ & $\ldots$ & $5.73 \mathrm{E}-15$ & 3.E-16 & $7.78 \mathrm{E}-15$ & 7.E-16 \\
\hline IRAS F09111-1007 & 2MASX J09133644-1019296 & $09^{\mathrm{h}} 13^{\mathrm{m}} 36^{\mathrm{s}} .50$ & $-10^{\circ} 19^{\prime} 29^{\prime \prime} .7$ & 11.90 & $3.10 \mathrm{E}-17$ & 1.E-17 & $9.30 \mathrm{E}-17$ & 8.E-18 \\
\hline IRAS F09111-1007 & 2MASX J09133888-1019196 & $09^{\mathrm{h}} 13^{\mathrm{m}} 38^{\mathrm{s}} .89$ & $-10^{\circ} 19^{\prime} 19^{\prime \prime} .6$ & 11.24 & $1.10 \mathrm{E}-15$ & 3.E-17 & $1.75 \mathrm{E}-15$ & 4.E-19 \\
\hline UGC 04881 & UGC 04881 NED02 & $09^{\mathrm{h}} 15^{\mathrm{m}} 54^{\mathrm{s}} .69$ & $+44^{\circ} 19^{\prime} 50^{\prime \prime} 8$ & 11.26 & $6.89 \mathrm{E}-17$ & 6.E-18 & $9.72 \mathrm{E}-17$ & 3.E-18 \\
\hline UGC 04881 & UGC 04881 NED01 & $09^{\mathrm{h}} 15^{\mathrm{m}} 55^{\mathrm{s}} .52$ & $+44^{\circ} 19^{\prime} 57^{\prime \prime} .4$ & 11.56 & $1.69 \mathrm{E}-16$ & 9.E-18 & $2.34 \mathrm{E}-16$ & 4.E-18 \\
\hline CGCG 239-011 & CGCG 239-011 NED01 & $09^{\mathrm{h}} 36^{\mathrm{m}} 30^{\mathrm{s}} .86$ & $+48^{\circ} 28^{\prime} 09^{\prime \prime} 9$ & 9.93 & $6.88 \mathrm{E}-15$ & 3.E-16 & $3.84 \mathrm{E}-15$ & 3.E-16 \\
\hline CGCG 239-011 & MCG+08-18-013 & $09^{\mathrm{h}} 36^{\mathrm{m}} 37^{\mathrm{s}} .20$ & $+48^{\circ} 28^{\prime} 27^{\prime \prime} .7$ & 11.32 & $2.88 \mathrm{E}-15$ & 2.E-16 & $2.65 \mathrm{E}-15$ & 3.E-16 \\
\hline GOALS J112110.3-025922 & 2MASX J11210825-0259399 & $11^{\mathrm{h}} 21^{\mathrm{m}} 08^{\mathrm{s}} .29$ & $-02^{\circ} 59^{\prime} 39^{\prime \prime} .2$ & 10.02 & $2.98 \mathrm{E}-16$ & 1.E-16 & $2.40 \mathrm{E}-16$ & 3.E-16 \\
\hline GOALS J112110.3-025922 & CGCG 011-076 & $11^{\mathrm{h}} 21^{\mathrm{m}} 12^{\mathrm{s}} \cdot 24$ & $-02^{\circ} 59^{\prime} 02^{\prime \prime} .5$ & 11.41 & $2.92 \mathrm{E}-16$ & 1.E-16 & 7.01E-16 & 3.E-16 \\
\hline IC 2810 & IC $2810 A$ & $11^{\mathrm{h}} 25^{\mathrm{m}} 45^{\mathrm{s}} .07$ & $+14^{\circ} 40^{\prime} 36^{\prime \prime} 0$ & 11.45 & $1.43 \mathrm{E}-15$ & 1.E-16 & $2.10 \mathrm{E}-15$ & 3.E-16 \\
\hline IC 2810 & IC 2810B & $11^{\mathrm{h}} 25^{\mathrm{m}} 49^{\mathrm{s}} .55$ & $+14^{\circ} 40^{\prime} 06^{\prime \prime} 6$ & 11.20 & $1.28 \mathrm{E}-16$ & 7.E-17 & $1.62 \mathrm{E}-16$ & 2.E-16 \\
\hline Arp 299 & NGC 3690 & $11^{\mathrm{h}} 28^{\mathrm{m}} 31^{\mathrm{s}} .04$ & $+58^{\circ} 33^{\prime} 40^{\prime \prime} .5$ & 11.77 & 8.32E-14 & 9.E-16 & $5.79 \mathrm{E}-14$ & 3.E-16 \\
\hline Arp 299 & IC 0694 & $11^{\mathrm{h}} 28^{\mathrm{m}} 33^{\mathrm{s}} \cdot 67$ & $+58^{\circ} 33^{\prime} 46^{\prime \prime} .1$ & 11.41 & $3.59 \mathrm{E}-14$ & 4.E-16 & $2.30 \mathrm{E}-14$ & 1.E-16 \\
\hline ESO 440-IG058 & ESO 440-IG058 NED01 & $12^{\mathrm{h}} 06^{\mathrm{m}} 51^{\mathrm{s}} .70$ & $-31^{\circ} 56^{\prime} 46^{\prime \prime} 4$ & 10.54 & $1.63 \mathrm{E}-15$ & 2.E-16 & $2.43 \mathrm{E}-15$ & 9.E-17 \\
\hline ESO 440-IG058 & ESO 440-IG058 NED02 & $12^{\mathrm{h}} 06^{\mathrm{m}} 51^{\mathrm{s}} .87$ & $-31^{\circ} 56^{\prime} 59^{\prime \prime} .2$ & 11.37 & $6.34 \mathrm{E}-16$ & 9.E-17 & $1.05 \mathrm{E}-15$ & 4.E-17 \\
\hline SGC 1211-470 & ESO 267-G029 & $12^{\mathrm{h}} 13^{\mathrm{m}} 52^{\mathrm{s}} .28$ & $-47^{\circ} 16^{\prime} 25^{\prime \prime} .4$ & 11.18 & $5.66 \mathrm{E}-15$ & 5.E-16 & $4.23 \mathrm{E}-15$ & 9.E-16 \\
\hline SGC 1211-470 & ESO 267-G030 & $12^{\mathrm{h}} 14^{\mathrm{m}} 12^{\mathrm{s}} .81$ & $-47^{\circ} 13^{\prime} 42^{\prime \prime} .5$ & 11.23 & $2.29 \mathrm{E}-15$ & 4.E-16 & $2.23 \mathrm{E}-15$ & 8.E-16 \\
\hline NGC 4922 & NGC 4922 NED01 & $13^{\mathrm{h}} 01^{\mathrm{m}} 24^{\mathrm{s}} .51$ & $+29^{\circ} 18^{\prime} 29^{\prime \prime} .8$ & 8.87 & $3.55 \mathrm{E}-16$ & 1.E-17 & $8.25 \mathrm{E}-16$ & 6.E-18 \\
\hline NGC 4922 & NGC 4922 NED02 & $13^{\mathrm{h}} 01^{\mathrm{m}} 25^{\mathrm{s}} .27$ & $+29^{\circ} 18^{\prime} 49^{\prime \prime} .5$ & 11.38 & $1.04 \mathrm{E}-15$ & 3.E-17 & $1.37 \mathrm{E}-15$ & 1.E-17 \\
\hline MCG-02-33-098-9 & MCG-02-33-098 & $13^{\mathrm{h}} 02^{\mathrm{m}} 19^{\mathrm{s}} .66$ & $-15^{\circ} 46^{\prime} 04^{\prime \prime} .2$ & 11.00 & $1.39 \mathrm{E}-15$ & 9.E-17 & $3.22 \mathrm{E}-15$ & 7.E-17 \\
\hline MCG-02-33-098-9 & MCG-02-33-099 & $13^{\mathrm{h}} 02^{\mathrm{m}} 20^{\mathrm{s}} .38$ & $-15^{\circ} 45^{\prime} 59^{\prime \prime} 6$ & 10.66 & $4.11 \mathrm{E}-15$ & 3.E-16 & $3.89 \mathrm{E}-15$ & 8.E-17 \\
\hline VV 250 & VV 250b & $13^{\mathrm{h}} 15^{\mathrm{m}} 30^{\mathrm{s}} .69$ & $+62^{\circ} 07^{\prime} 45^{\prime \prime} .8$ & $\ldots$ & $2.92 \mathrm{E}-15$ & 1.E-16 & $2.80 \mathrm{E}-15$ & 4.E-17 \\
\hline VV 250 & VV 250a & $13^{\mathrm{h}} 15^{\mathrm{m}} 34^{\mathrm{s}} .96$ & $+62^{\circ} 07^{\prime} 29^{\prime \prime} .2$ & $\ldots$ & $5.74 \mathrm{E}-15$ & 3.E-16 & $6.06 \mathrm{E}-15$ & 1.E-16 \\
\hline NGC 5256 & MRK 266B & $13^{\mathrm{h}} 38^{\mathrm{m}} 17^{\mathrm{s}} .25$ & $+48^{\circ} 16^{\prime} 32^{\prime \prime} .9$ & 11.37 & $4.38 \mathrm{E}-15$ & 2.E-16 & $4.00 \mathrm{E}-15$ & 7.E-17 \\
\hline NGC 5256 & MRK 266A & $13^{\mathrm{h}} 38^{\mathrm{m}} 17^{\mathrm{s}} .79$ & $+48^{\circ} 16^{\prime} 41^{\prime \prime} 6$ & 11.11 & $5.80 \mathrm{E}-15$ & 2.E-16 & $5.45 \mathrm{E}-15$ & 9.E-17 \\
\hline Arp 240 & NGC 5257 & $13^{\mathrm{h}} 39^{\mathrm{m}} 52^{\mathrm{s}} .95$ & $+00^{\circ} 50^{\prime} 25^{\prime \prime} .9$ & 11.31 & $1.10 \mathrm{E}-14$ & 4.E-16 & $9.63 \mathrm{E}-15$ & 4.E-16 \\
\hline Arp 240 & NGC 5258 & $13^{\mathrm{h}} 39^{\mathrm{m}} 57^{\mathrm{s}} .72$ & $+00^{\circ} 49^{\prime} 53^{\prime \prime} .0$ & 11.32 & $2.84 \mathrm{E}-14$ & 6.E-16 & $2.11 \mathrm{E}-14$ & 5.E-16 \\
\hline NGC 5331 & NGC 5331 NED01 & $13^{\mathrm{h}} 52^{\mathrm{m}} 16^{\mathrm{s}} .21$ & $+02^{\circ} 06^{\prime} 05^{\prime \prime} .1$ & 11.54 & $1.32 \mathrm{E}-15$ & 2.E-17 & $1.59 \mathrm{E}-15$ & 6.E-18 \\
\hline NGC 5331 & NGC 5331 NED02 & $13^{\mathrm{h}} 52^{\mathrm{m}} 16^{\mathrm{s}} .43$ & $+02^{\circ} 06^{\prime} 30^{\prime} .9$ & 11.02 & $3.89 \mathrm{E}-15$ & 5.E-17 & $3.64 \mathrm{E}-15$ & 1.E-17 \\
\hline GOALS J144510.0-205331 & NGC 5734 & $14^{\mathrm{h}} 45^{\mathrm{m}} 09^{\mathrm{s}} .04$ & $-20^{\circ} 52^{\prime} 13^{\prime \prime} .2$ & 11.04 & $2.64 \mathrm{E}-15$ & 3.E-16 & $4.03 \mathrm{E}-15$ & 7.E-16 \\
\hline GOALS J144510.0-205331 & NGC 5743 & $14^{\mathrm{h}} 45^{\mathrm{m}} 11^{\mathrm{s}} .02$ & $-20^{\circ} 54^{\prime} 48^{\prime \prime} .6$ & 10.86 & $1.06 \mathrm{E}-14$ & 4.E-16 & $9.61 \mathrm{E}-15$ & 7.E-16 \\
\hline
\end{tabular}


Table 2

(Continued)

\begin{tabular}{|c|c|c|c|c|c|c|c|c|}
\hline $\begin{array}{c}\text { System Name } \\
\text { (1) }\end{array}$ & $\begin{array}{c}\text { Galaxy Name } \\
(2)\end{array}$ & $\begin{array}{c}\text { R.A. (J2000) } \\
(3)\end{array}$ & $\begin{array}{c}\text { Decl. (J2000) } \\
(4) \\
\end{array}$ & $\begin{array}{c}\log \left(L_{\mathrm{IR}}\right) \\
(5)\end{array}$ & $\begin{array}{c}f_{v}(\mathrm{FUV}) \\
(6)\end{array}$ & $\begin{array}{c}\sigma_{\mathrm{FUV}} \\
(7)\end{array}$ & $\begin{array}{c}f_{v}(\mathrm{NUV}) \\
(8) \\
\end{array}$ & $\begin{array}{c}\sigma_{\mathrm{NUV}} \\
(9) \\
\end{array}$ \\
\hline VV 340 & VV 340b & $14^{\mathrm{h}} 57^{\mathrm{m}} 00^{\mathrm{s}} 32$ & $+24^{\circ} 36^{\prime} 24^{\prime \prime} .6$ & 10.83 & $3.48 \mathrm{E}-15$ & 4.E-17 & $2.96 \mathrm{E}-15$ & 1.E-17 \\
\hline VV 340 & VV 340a & $14^{\mathrm{h}} 57^{\mathrm{m}} 00^{\mathrm{s}} .70$ & $+24^{\circ} 37^{\prime} 05^{\prime \prime} .8$ & 11.69 & $1.02 \mathrm{E}-15$ & 2.E-17 & $8.53 \mathrm{E}-16$ & 6.E-18 \\
\hline Arp 293 & NGC 6285 & $16^{\mathrm{h}} 58^{\mathrm{m}} 23^{\mathrm{s}} .99$ & $+58^{\circ} 57^{\prime} 21^{\prime \prime} .7$ & 10.72 & $3.80 \mathrm{E}-15$ & 2.E-16 & $3.47 \mathrm{E}-15$ & 3.E-16 \\
\hline Arp 293 & NGC 6286 & $16^{\mathrm{h}} 58^{\mathrm{m}} 31^{\mathrm{s}} .63$ & $+58^{\circ} 56^{\prime} 13^{\prime \prime} .3$ & 11.36 & $3.52 \mathrm{E}-15$ & 2.E-16 & $2.99 \mathrm{E}-15$ & 3.E-16 \\
\hline UGC 11175 & NGC 6621 & $18^{\mathrm{h}} 12^{\mathrm{m}} 55^{\mathrm{s}} .39$ & $+68^{\circ} 21^{\prime} 48^{\prime \prime} .2$ & 11.28 & $1.86 \mathrm{E}-15$ & $9 . \mathrm{E}-17$ & $2.93 \mathrm{E}-15$ & 5.E-17 \\
\hline UGC 11175 & NGC 6621 SE & $18^{\mathrm{h}} 12^{\mathrm{m}} 58^{\mathrm{s}} .52$ & $+68^{\circ} 21^{\prime} 29^{\prime \prime} .4$ & 9.52 & $3.63 \mathrm{E}-15$ & 2.E-16 & $2.73 \mathrm{E}-15$ & 5.E-17 \\
\hline UGC 11175 & NGC 6622 & $18^{\mathrm{h}} 12^{\mathrm{m}} 59^{\mathrm{s}} .68$ & $+68^{\circ} 21^{\prime} 15^{\prime \prime} .1$ & 9.23 & $1.48 \mathrm{E}-16$ & 7.E-18 & $4.79 \mathrm{E}-16$ & 9.E-18 \\
\hline KTS57 & IC 4686 & $18^{\mathrm{h}} 13^{\mathrm{m}} 38^{\mathrm{s}} .77$ & $-57^{\circ} 43^{\prime} 57^{\prime \prime} .3$ & 11.02 & $1.13 \mathrm{E}-14$ & 3.E-16 & $1.08 \mathrm{E}-14$ & 1.E-16 \\
\hline KTS57 & IC 4687 & $18^{\mathrm{h}} 13^{\mathrm{m}} 39^{\mathrm{s}} .80$ & $-57^{\circ} 43^{\prime} 30^{\prime \prime} .7$ & 11.32 & $4.11 \mathrm{E}-15$ & 1.E-16 & $3.52 \mathrm{E}-15$ & 4.E-17 \\
\hline KTS57 & IC 4689 & $18^{\mathrm{h}} 13^{\mathrm{m}} 40^{\mathrm{s}} .38$ & $-57^{\circ} 44^{\prime} 54^{\prime \prime} .3$ & 11.02 & $1.92 \mathrm{E}-15$ & 2.E-16 & $1.51 \mathrm{E}-15$ & 4.E-16 \\
\hline NGC 6670AB & NGC 6670B & $18^{\mathrm{h}} 33^{\mathrm{m}} 32^{\mathrm{s}} .78$ & $+59^{\circ} 53^{\prime} 11^{\prime \prime} .7$ & 11.32 & $7.77 \mathrm{E}-16$ & 2.E-17 & $8.71 \mathrm{E}-16$ & 9.E-18 \\
\hline NGC 6670AB & NGC 6670A & $18^{\mathrm{h}} 33^{\mathrm{m}} 37^{\mathrm{s}} .67$ & $+59^{\circ} 53^{\prime} 21^{\prime \prime} .3$ & 11.38 & $1.86 \mathrm{E}-15$ & 3.E-17 & $1.73 \mathrm{E}-15$ & 1.E-17 \\
\hline ESO 343-IG013 & ESO 343-IG013 NED01 & $21^{\mathrm{h}} 36^{\mathrm{m}} 10^{\mathrm{s}} .53$ & $-38^{\circ} 32^{\prime} 42^{\prime \prime} .8$ & 10.60 & $2.63 \mathrm{E}-15$ & 2.E-16 & $2.76 \mathrm{E}-15$ & 9.E-17 \\
\hline ESO 343-IG013 & ESO 343-IG013 NED02 & $21^{\mathrm{h}} 36^{\mathrm{m}} 10^{\mathrm{s}} .93$ & $-38^{\circ} 32^{\prime} 33^{\prime \prime} .0$ & 10.99 & $1.32 \mathrm{E}-15$ & 1.E-16 & $1.49 \mathrm{E}-15$ & 5.E-17 \\
\hline Arp 298 & NGC 7469 & $23^{\mathrm{h}} 03^{\mathrm{m}} 15^{\mathrm{s}} \cdot 64$ & $+08^{\circ} 52^{\prime} 25^{\prime \prime} .5$ & 11.58 & $4.32 \mathrm{E}-14$ & 1.E-16 & $3.11 \mathrm{E}-14$ & 1.E-16 \\
\hline Arp 298 & IC 5283 & $23^{\mathrm{h}} 03^{\mathrm{m}} 18^{\mathrm{s}} .04$ & $+08^{\circ} 53^{\prime} 36^{\prime \prime} .5$ & 10.79 & $1.77 \mathrm{E}-15$ & 3.E-17 & $1.76 \mathrm{E}-15$ & 5.E-17 \\
\hline NGC 7592 & NGC 7592A & $23^{\mathrm{h}} 18^{\mathrm{m}} 21^{\mathrm{s}} .78$ & $-04^{\circ} 24^{\prime} 57^{\prime \prime} .0$ & 11.17 & $2.35 \mathrm{E}-15$ & $6 . \mathrm{E}-17$ & $1.66 \mathrm{E}-15$ & 2.E-17 \\
\hline NGC 7592 & NGC 7592B & $23^{\mathrm{h}} 18^{\mathrm{m}} 22^{\mathrm{s}} .60$ & $-04^{\circ} 24^{\prime} 58^{\prime \prime} 0$ & 11.01 & $2.09 \mathrm{E}-14$ & 5.E-16 & $1.21 \mathrm{E}-14$ & 2.E-16 \\
\hline ESO 077-IG014 & ESO 077-IG014 NED01 & $23^{\mathrm{h}} 21^{\mathrm{m}} 03^{\mathrm{s}} .73$ & $-69^{\circ} 13^{\prime} 01^{\prime \prime} .0$ & 11.33 & $5.08 \mathrm{E}-16$ & 4.E-17 & $8.42 \mathrm{E}-16$ & 9.E-18 \\
\hline ESO 077-IG014 & ESO 077-IG014 NED02 & $23^{\mathrm{h}} 21^{\mathrm{m}} 05^{\mathrm{s}} .45$ & $-69^{\circ} 12^{\prime} 47^{\prime \prime} .3$ & 11.56 & $2.64 \mathrm{E}-17$ & 2.E-18 & $1.91 \mathrm{E}-16$ & 2.E-18 \\
\hline Arp 182 & NGC 7674 & $23^{\mathrm{h}} 27^{\mathrm{m}} 56^{\mathrm{s}} .71$ & $+08^{\circ} 46^{\prime} 44^{\prime \prime} .3$ & 11.55 & $1.30 \mathrm{E}-14$ & 1.E-16 & $9.96 \mathrm{E}-15$ & 4.E-17 \\
\hline Arp 182 & NGC 7674A & $23^{\mathrm{h}} 27^{\mathrm{m}} 58^{\mathrm{s}} .77$ & $+08^{\circ} 46^{\prime} 57^{\prime \prime} .9$ & 10.01 & $1.23 \mathrm{E}-15$ & 1.E-17 & $1.64 \mathrm{E}-15$ & 6.E-18 \\
\hline GOALS J2341454-033944 & MRK 933 & $23^{\mathrm{h}} 41^{\mathrm{m}} 43^{\mathrm{s}} .69$ & $-03^{\circ} 39^{\prime} 26^{\prime \prime} .5$ & 10.17 & $3.02 \mathrm{E}-15$ & 6.E-17 & $2.88 \mathrm{E}-15$ & 9.E-17 \\
\hline GOALS J2341454-033944 & MCG-01-60-021 & $23^{\mathrm{h}} 41^{\mathrm{m}} 47^{\mathrm{s}} .33$ & $-03^{\circ} 40^{\prime} 01^{\prime \prime} 7$ & 10.43 & $3.58 \mathrm{E}-16$ & 3.E-17 & $\ldots$ & $\ldots$ \\
\hline GOALS J2341454-033944 & MCG-01-60-022 & $23^{\mathrm{h}} 42^{\mathrm{m}} 00^{\mathrm{s}} .91$ & $-03^{\circ} 36^{\prime} 54^{\prime \prime} .4$ & 11.15 & $7.99 \mathrm{E}-15$ & 1.E-16 & $6.20 \mathrm{E}-15$ & 2.E-16 \\
\hline KTG 82 & NGC 7769 & $23^{\mathrm{h}} 51^{\mathrm{m}} 03^{\mathrm{s}} .91$ & $+20^{\circ} 09^{\prime} 01^{\prime \prime} .7$ & 10.74 & $3.39 \mathrm{E}-14$ & 8.E-17 & $2.35 \mathrm{E}-14$ & 6.E-17 \\
\hline KTG 82 & NGC 7770 & $23^{\mathrm{h}} 51^{\mathrm{m}} 22^{\mathrm{s}} .55$ & $+20^{\circ} 05^{\prime} 49^{\prime \prime} .2$ & 10.67 & $1.07 \mathrm{E}-14$ & 4.E-17 & $7.63 \mathrm{E}-15$ & 3.E-17 \\
\hline KTG 82 & NGC 7771 & $23^{\mathrm{h}} 51^{\mathrm{m}} 24^{\mathrm{s}} .80$ & $+20^{\circ} 06^{\prime} 42^{\prime \prime} .2$ & 11.17 & $5.13 \mathrm{E}-15$ & 3.E-17 & $6.39 \mathrm{E}-15$ & 3.E-17 \\
\hline
\end{tabular}

Note. Column 1: system name; Column 2: galaxy name; Column 3: the right ascension (J2000) of the IRAC $8 \mu \mathrm{m}$ centroid in J. Mazzarella et al. (2010, in preparation); Column 4: the declination (J2000) of the IRAC $8 \mu \mathrm{m}$ centroid in J. Mazzarella et al. (2010, in preparation); Column 5: the infrared luminosity in $\log _{10}$ Solar units computed using the MIPS flux densities reported in J. Mazzarella et al. (2010, in preparation) and the luminosity distances in Armus et al. (2009); see the text for details; Column 6: GALEX FUV flux density in units of $\mathrm{erg} \mathrm{s}^{-1} \mathrm{~cm}^{-2} \AA^{-1}$; Column 7: GALEX FUV flux density uncertainty; Column 8: GALEX NUV flux density in units of $\mathrm{erg} \mathrm{s}^{-1} \mathrm{~cm}^{-2} \AA^{-1}$; Column 9: GALEX NUV flux density uncertainty.
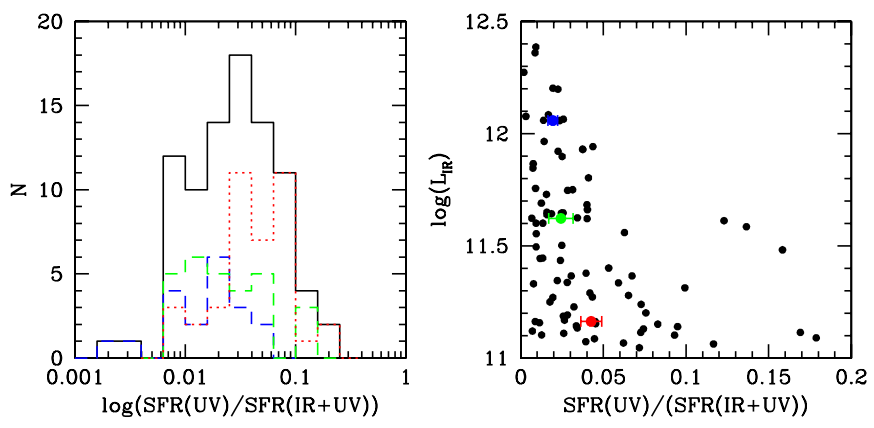

Figure 2. Left: histogram showing the ratio of SFR(UV) to SFR(UV+IR). The solid line is the full GOALS GALEX sample. Colored lines show the GOALS GALEX sample divided into luminosity bins as in Figure 1: $10^{11} L_{\odot}<L_{\mathrm{IR}}<$ $10^{11.4} L_{\odot}$ (red dotted line), $10^{11.4} L_{\odot}<L_{\mathrm{IR}}<10^{11.8} L_{\odot}$ (green dashed line), and $L_{\mathrm{IR}}>10^{11.8} L_{\odot}$ (blue dashed line). The FUV contribution to SFR is small for (U)LIRGs and decreases with increasing $\mathrm{L}_{\mathrm{IR}}$. Right: $L_{\mathrm{IR}}$ plotted against the ratio of SFR(UV) to SFR(UV+IR). Median ratios of the star formation rates are shown for each luminosity bin (red: $10^{11} L_{\odot}<L_{I \mathrm{R}}<10^{11.4} L_{\odot}$, green: $10^{11.4} L_{\odot}<L_{\mathrm{IR}}<10^{11.8} L_{\odot}$, blue: $L_{\mathrm{IR}}>10^{11.8} L_{\odot}$ ) along with $1 \sigma$ standard deviations of the mean. Although anti-correlated (Spearman rank correlation coefficient of -0.47), the correlation is not linear.

derived from $K$-band data were used where possible. For the galaxies without reliable $K$-band photometry, the masses estimated from $3.6 \mu \mathrm{m}$ data were scaled by the median ratio of $\operatorname{mass}(K) / \operatorname{mass}(3.6)$ from galaxies measured at both wavelengths. Stellar masses range from $4.3 \times 10^{10}$ to $6.4 \times 10^{11} M_{\odot}$, with a median of $1.4 \times 10^{11} M_{\odot}$ and a mean of $(1.63 \pm 0.09) \times$ $10^{11} M_{\odot}$. The specific star formation rate (SSFR; SFR per unit mass) ranges from $5.5 \times 10^{-11}$ to $3.5 \times 10^{-9} \mathrm{yr}^{-1}$, with a median of $3.9 \times 10^{-10} \mathrm{yr}^{-1}$ and a mean of $(6.2 \pm 0.7) \times 10^{-10} \mathrm{yr}^{-1}$. These correspond to mass doubling timescales of $18 \mathrm{Gyr}$ to 290 Myr, with a median of $2.6 \mathrm{Gyr}$.

The Spitzer Infrared Nearby Galaxy Survey (SINGS; Kennicutt et al. 2003) provides a useful comparison sample of lower luminosity galaxies observed with both GALEX and Spitzer. The upper bound of the SSFRs measured for SINGS galaxies is approximately $3 \times 10^{-10} \mathrm{yr}^{-1}$ (Dale et al. 2007). The IR/UV ratio, a useful observational measure of dust extinction, is defined as

$$
\mathrm{IR} / \mathrm{UV}=\frac{L_{\mathrm{IR}}}{v L_{v}(\mathrm{FUV})+v L_{v}(\mathrm{NUV})}
$$

and ranges from 5.8 to 813 , with a median of 39 . Figure 3 compares the IR/UV ratio against SSFR for both the GOALS and SINGS samples (Dale et al. 2007). In GOALS systems, the IR/UV ratio is correlated with SSFR $\left(r_{s}=0.55\right.$, significance $2 \times 10^{-8}$ ), with large scatter: LIRGs and ULIRGs with high SSFR also have high IR/UV ratios. The two quantities are anticorrelated $\left(r_{s}=-0.61\right.$, significance $\left.1 \times 10^{-6}\right)$ for SINGS galaxies with SSFR $>10^{-11} \mathrm{yr}^{-1}$. A handful of SINGS galaxies have IR/UV ratios which are as high as seen in the GOALS sample, but their SSFRs are significantly lower. Taken 
Table 3

Derived Quantities-Integrated Systems

\begin{tabular}{|c|c|c|c|c|c|c|c|c|c|}
\hline System & $\log \left(L_{\mathrm{FUV}}\right)$ & $\beta(G A L E X)$ & $\sigma_{\beta(G A L E X)}$ & $\begin{array}{l}\text { IRX } \\
(5)\end{array}$ & $\begin{array}{c}\sigma_{\text {IRX }} \\
(6)\end{array}$ & $\begin{array}{c}\text { Mass } \\
\left(M_{\odot}\right) \\
(7)\end{array}$ & $\begin{array}{c}\mathrm{SFR} \\
\left(M_{\odot} \mathrm{yr}^{-1}\right) \\
(8)\end{array}$ & $\begin{array}{c}\text { SSFR } \\
\left(\mathrm{yr}^{-1}\right) \\
(9)\end{array}$ & $\begin{array}{c}\text { IR/UV } \\
(10)\end{array}$ \\
\hline NGC 0023 & 9.39 & 0.409 & 0.016 & 1.724 & 0.003 & $1.59 \mathrm{E}+11$ & 23.34 & $1.47 \mathrm{E}-10$ & 19.2 \\
\hline NGC 0034 & 9.69 & -0.907 & 0.078 & 1.799 & 0.013 & $1.10 \mathrm{E}+11$ & {$[55.25]$} & [5.04E-10] & 31.2 \\
\hline Arp 256 & 10.22 & -0.139 & 0.016 & 1.262 & 0.003 & $1.80 \mathrm{E}+11$ & 57.11 & $3.18 \mathrm{E}-10$ & 7.6 \\
\hline ESO 350-IG038 & 10.15 & -1.281 & 0.009 & 1.128 & 0.001 & $4.63 \mathrm{E}+10$ & [37.02] & [7.99E-10] & 7.2 \\
\hline NGC 0232 & 9.04 & -0.265 & 0.224 & 2.404 & 0.037 & $1.94 \mathrm{E}+11$ & 48.65 & $2.50 \mathrm{E}-10$ & 108.7 \\
\hline MCG+12-02-001 & 7.90 & -1.915 & 2.361 & 3.593 & 0.343 & $8.10 \mathrm{E}+10$ & 54.49 & $6.73 \mathrm{E}-10$ & 2348.3 \\
\hline NGC 0317B & 8.82 & 1.421 & 0.339 & 2.367 & 0.060 & $1.16 \mathrm{E}+11$ & 26.97 & $2.33 \mathrm{E}-10$ & 63.0 \\
\hline IC 1623 & 10.33 & -0.649 & 0.010 & 1.379 & 0.002 & $1.62 \mathrm{E}+11$ & [94.09] & [5.80E-10] & 11.2 \\
\hline MCG-03-04-014 & 9.52 & 0.100 & 0.034 & 2.127 & 0.006 & $1.64 \mathrm{E}+11$ & 78.53 & $4.80 \mathrm{E}-10$ & 52.5 \\
\hline ESO 244-G012 & 9.53 & -0.376 & 0.104 & 1.848 & 0.017 & $1.37 \mathrm{E}+11$ & 42.66 & $3.11 \mathrm{E}-10$ & 31.1 \\
\hline CGCG 436-030 & 9.71 & 0.721 & 0.023 & 1.974 & 0.004 & $7.12 \mathrm{E}+10$ & {$[85.87]$} & [1.21E-09] & 31.3 \\
\hline IRAS F01364-1042 & 8.71 & 2.662 & 0.284 & 3.136 & 0.051 & $4.33 \mathrm{E}+10$ & 122.61 & 2.83E-09 & 247.6 \\
\hline NGC 0695 & 9.63 & 0.764 & 0.029 & 2.053 & 0.005 & $2.01 \mathrm{E}+11$ & 84.64 & $4.21 \mathrm{E}-10$ & 37.1 \\
\hline UGC 01385 & 9.50 & -0.560 & 0.090 & 1.546 & 0.015 & $6.05 \mathrm{E}+10$ & 20.32 & $3.36 \mathrm{E}-10$ & 16.2 \\
\hline NGC 0877 & 9.59 & -0.118 & 0.015 & 1.512 & 0.002 & $1.61 \mathrm{E}+11$ & 22.83 & $1.41 \mathrm{E}-10$ & 13.5 \\
\hline MCG+05-06-036 & 9.33 & 1.029 & 0.192 & 2.316 & 0.034 & $2.88 \mathrm{E}+11$ & 77.18 & $2.68 \mathrm{E}-10$ & 63.0 \\
\hline NGC 0958 & 9.62 & -0.249 & 0.021 & 1.581 & 0.004 & $2.79 \mathrm{E}+11$ & 28.71 & $1.03 \mathrm{E}-10$ & 16.3 \\
\hline NGC 1068 & 9.53 & -0.194 & 0.005 & 1.865 & 0.001 & $1.44 \mathrm{E}+11$ & [44.29] & [3.07E-10] & 30.9 \\
\hline UGC 02238 & 8.74 & -0.233 & 0.134 & 2.591 & 0.022 & $8.71 \mathrm{E}+10$ & 37.70 & $4.33 \mathrm{E}-10$ & 166.2 \\
\hline NGC 1275 & 9.67 & -0.147 & 0.007 & 1.593 & 0.001 & $4.29 \mathrm{E}+11$ & [32.78] & [7.65E-11] & 16.4 \\
\hline NGC 1365 & 9.24 & -0.203 & 0.012 & 1.760 & 0.002 & $1.44 \mathrm{E}+11$ & {$[17.83]$} & [1.24E-10] & 24.4 \\
\hline IRAS F03359+1523 & 8.98 & 0.660 & 0.873 & 2.562 & 0.157 & $5.56 \mathrm{E}+10$ & 61.22 & 1.10E-09 & 123.2 \\
\hline CGCG 465-012 & 8.14 & 3.748 & 2.176 & 3.054 & 0.395 & $1.17 \mathrm{E}+11$ & 27.51 & $2.35 \mathrm{E}-10$ & 139.2 \\
\hline UGC 02982 & 8.06 & -1.453 & 2.293 & 3.141 & 0.363 & $8.57 \mathrm{E}+10$ & 27.71 & $3.23 \mathrm{E}-10$ & 764.9 \\
\hline ESO 550-IG025 & 9.36 & 0.107 & 0.240 & 2.142 & 0.041 & $1.14 \mathrm{E}+11$ & 56.46 & $4.95 \mathrm{E}-10$ & 54.3 \\
\hline ESO 203-IG001 & 9.26 & -0.195 & 0.112 & 2.606 & 0.019 & $7.33 \mathrm{E}+10$ & 127.43 & $1.74 \mathrm{E}-09$ & 170.5 \\
\hline MCG-05-12-006 & 8.69 & 2.091 & 0.388 & 2.479 & 0.069 & $4.39 \mathrm{E}+10$ & 26.06 & $5.93 \mathrm{E}-10$ & 65.9 \\
\hline CGCG 468-002 & 8.32 & -0.940 & 1.609 & 2.900 & 0.269 & $8.37 \mathrm{E}+10$ & [29.15] & [3.48E-10] & 396.5 \\
\hline IRAS $05083+2441$ & 7.95 & 1.277 & 2.568 & 3.313 & 0.460 & $7.86 \mathrm{E}+10$ & 31.95 & $4.06 \mathrm{E}-10$ & 581.5 \\
\hline IRAS $05129+5128$ & 8.54 & 0.244 & 1.422 & 2.882 & 0.248 & $5.07 \mathrm{E}+10$ & 45.90 & $9.05 \mathrm{E}-10$ & 288.1 \\
\hline IRAS F05189-2524 & 9.38 & -0.362 & 0.130 & 2.783 & 0.022 & $3.19 \mathrm{E}+11$ & {$[253.48]$} & [7.95E-10] & 266.5 \\
\hline NGC 1961 & 9.66 & -0.146 & 0.011 & 1.403 & 0.002 & $3.92 \mathrm{E}+11$ & 21.38 & $5.45 \mathrm{E}-11$ & 10.6 \\
\hline UGC 03410 & 8.72 & -0.199 & 0.218 & 2.383 & 0.037 & $1.23 \mathrm{E}+11$ & 22.03 & $1.79 \mathrm{E}-10$ & 102.0 \\
\hline NGC 2146 & 8.30 & 0.789 & 0.025 & 2.820 & 0.004 & $6.41 \mathrm{E}+10$ & 22.87 & $3.57 \mathrm{E}-10$ & 215.2 \\
\hline ESO 255-IG007 & 9.85 & -0.110 & 0.030 & 2.048 & 0.005 & $1.80 \mathrm{E}+11$ & 140.00 & 7.79E-10 & 46.1 \\
\hline NGC 2342 & 9.86 & -0.274 & 0.063 & 1.453 & 0.011 & $1.97 \mathrm{E}+11$ & 37.60 & $1.90 \mathrm{E}-10$ & 12.2 \\
\hline NGC 2369 & 8.73 & -0.199 & 0.213 & 2.427 & 0.036 & $1.24 \mathrm{E}+11$ & 25.00 & $2.01 \mathrm{E}-10$ & 113.0 \\
\hline NGC 2388 & 9.72 & -0.721 & 0.015 & 1.559 & 0.002 & $1.43 \mathrm{E}+11$ & 34.74 & $2.43 \mathrm{E}-10$ & 17.3 \\
\hline MCG+02-20-003 & 9.45 & -0.563 & 0.096 & 1.680 & 0.016 & $8.22 \mathrm{E}+10$ & [24.22] & [2.95E-10] & 22.0 \\
\hline NGC 2623 & 9.25 & -0.234 & 0.038 & 2.351 & 0.006 & $6.42 \mathrm{E}+10$ & 69.19 & 1.08E-09 & 95.6 \\
\hline ESO 060-IG016 & 9.32 & 0.945 & 0.238 & 2.495 & 0.042 & $9.37 \mathrm{E}+10$ & {$[115.40]$} & [1.23E-09] & 97.5 \\
\hline IRAS F08572+3915 & 9.62 & 0.791 & 0.103 & 2.548 & 0.018 & $6.38 \mathrm{E}+11$ & [254.32] & [3.98E-10] & 115.1 \\
\hline IRAS 09022-3615 & 8.86 & 3.672 & 0.650 & 3.451 & 0.118 & $1.66 \mathrm{E}+11$ & [359.05] & [2.17E-09] & 356.6 \\
\hline IRAS F09111-1007 & 9.44 & 1.304 & 0.106 & 2.619 & 0.019 & $1.43 \mathrm{E}+11$ & 198.46 & $1.39 \mathrm{E}-09$ & 116.8 \\
\hline UGC 04881 & 9.55 & 0.726 & 0.040 & 2.197 & 0.007 & $2.59 \mathrm{E}+11$ & 97.13 & $3.76 \mathrm{E}-10$ & 52.3 \\
\hline UGC 05101 & 9.09 & 0.876 & 0.136 & 2.925 & 0.024 & $1.97 \mathrm{E}+11$ & [180.18] & [9.15E-10] & 267.6 \\
\hline MCG+08-18-013 & 9.78 & -0.974 & 0.100 & 1.555 & 0.016 & $7.42 \mathrm{E}+10$ & 39.55 & 5.33E-10 & 18.1 \\
\hline Arp 303 & 9.26 & -0.220 & 0.153 & 1.968 & 0.026 & $1.73 \mathrm{E}+11$ & 29.83 & $1.72 \mathrm{E}-10$ & 39.5 \\
\hline NGC 3110 & 9.72 & -0.183 & 0.085 & 1.646 & 0.014 & $1.32 \mathrm{E}+11$ & 41.72 & $3.15 \mathrm{E}-10$ & 18.6 \\
\hline ESO 374-IG032 & 9.39 & 2.969 & 0.054 & 2.394 & 0.010 & $2.89 \mathrm{E}+11$ & [106.13] & [3.67E-10] & 40.3 \\
\hline IRAS F10173+0828 & 7.10 & 12.181 & 11.648 & 4.760 & 2.118 & $3.67 \mathrm{E}+10$ & 126.32 & $3.44 \mathrm{E}-09$ & 235.0 \\
\hline NGC 3221 & 9.19 & 0.169 & 0.119 & 1.896 & 0.020 & $1.55 \mathrm{E}+11$ & 21.75 & $1.40 \mathrm{E}-10$ & 30.3 \\
\hline NGC 3256 & 9.34 & 0.433 & 0.018 & 2.299 & 0.003 & $1.14 \mathrm{E}+11$ & 76.46 & $6.71 \mathrm{E}-10$ & 71.6 \\
\hline ESO 264-G036 & 7.54 & 8.485 & 12.042 & 3.781 & 2.189 & $2.45 \mathrm{E}+11$ & 36.54 & $1.49 \mathrm{E}-10$ & 114.3 \\
\hline IRAS F10565+2448 & 8.83 & 1.997 & 0.237 & 3.247 & 0.042 & $1.49 \mathrm{E}+11$ & 209.13 & $1.40 \mathrm{E}-09$ & 398.4 \\
\hline MCG+07-23-019 & 9.86 & -0.804 & 0.111 & 1.761 & 0.020 & $9.36 \mathrm{E}+10$ & 75.13 & $8.03 \mathrm{E}-10$ & 28.0 \\
\hline CGCG 011-076 & 8.56 & 1.114 & 0.517 & 2.871 & 0.090 & $1.31 \mathrm{E}+11$ & 46.52 & $3.55 \mathrm{E}-10$ & 220.4 \\
\hline IRAS F11231+1456 & 9.23 & 0.897 & 0.240 & 2.413 & 0.041 & $1.84 \mathrm{E}+11$ & 76.35 & 4.15E-10 & 81.8 \\
\hline NGC 3690 & 10.16 & -0.923 & 0.029 & 1.771 & 0.005 & $1.55 \mathrm{E}+11$ & {$[150.55]$} & [9.74E-10] & 29.4 \\
\hline ESO 320-G030 & 8.47 & 0.633 & 0.255 & 2.693 & 0.045 & $4.63 \mathrm{E}+10$ & 25.64 & $5.54 \mathrm{E}-10$ & 168.0 \\
\hline ESO 440-IG058 & 9.11 & 1.027 & 0.336 & 2.325 & 0.059 & $1.24 \mathrm{E}+11$ & 47.53 & $3.83 \mathrm{E}-10$ & 64.4 \\
\hline IRAS F12112+0305 & 9.85 & -0.606 & 0.064 & 2.510 & 0.010 & $2.17 \mathrm{E}+11$ & 402.89 & $1.85 \mathrm{E}-09$ & 150.4 \\
\hline ESO 267-G030 & 8.99 & -0.060 & 0.473 & 2.260 & 0.082 & $2.57 \mathrm{E}+11$ & 31.31 & $1.22 \mathrm{E}-10$ & 74.4 \\
\hline NGC 4922 & 8.89 & 1.073 & 0.080 & 2.489 & 0.014 & $1.73 \mathrm{E}+11$ & [41.95] & [2.42E-10] & 92.6 \\
\hline
\end{tabular}


Table 3

(Continued)

\begin{tabular}{|c|c|c|c|c|c|c|c|c|c|}
\hline $\begin{array}{c}\text { System } \\
\text { (1) } \\
\end{array}$ & $\begin{array}{c}\log \left(L_{\mathrm{FUV}}\right) \\
(2)\end{array}$ & $\begin{array}{c}\beta(G A L E X) \\
(3)\end{array}$ & $\begin{array}{c}\sigma_{\beta(G A L E X)} \\
(4)\end{array}$ & $\begin{array}{l}\text { IRX } \\
\text { (5) } \\
\end{array}$ & $\begin{array}{c}\sigma_{\text {IRX }} \\
(6) \\
\end{array}$ & $\begin{array}{c}\text { Mass } \\
\left(M_{\odot}\right) \\
(7) \\
\end{array}$ & $\begin{array}{c}\mathrm{SFR} \\
\left(M_{\left.\odot \mathrm{yr}^{-1}\right)}\right) \\
(8)\end{array}$ & $\begin{array}{c}\text { SSFR } \\
\left(\mathrm{yr}^{-1}\right) \\
(9) \\
\end{array}$ & $\begin{array}{r}\text { IR/UV } \\
(10) \\
\end{array}$ \\
\hline CGCG 043-099 & 9.36 & -0.516 & 0.299 & 2.330 & 0.048 & $1.19 \mathrm{E}+11$ & 84.73 & $7.11 \mathrm{E}-10$ & 97.2 \\
\hline MCG-02-33-098-9 & 9.19 & 0.612 & 0.161 & 1.973 & 0.028 & $5.91 \mathrm{E}+10$ & 25.87 & $4.38 \mathrm{E}-10$ & 32.2 \\
\hline ESO 507-G070 & 9.01 & -0.040 & 0.115 & 2.544 & 0.020 & $1.23 \mathrm{E}+11$ & 62.77 & $5.12 \mathrm{E}-10$ & 142.1 \\
\hline VV 250a & 9.89 & 0.057 & 0.111 & 1.913 & 0.019 & $1.12 \mathrm{E}+11$ & 113.75 & $1.01 \mathrm{E}-09$ & 32.4 \\
\hline UGC 08387 & 9.35 & 0.284 & 0.156 & 2.379 & 0.026 & $6.72 \mathrm{E}+10$ & 94.18 & $1.40 \mathrm{E}-09$ & 89.6 \\
\hline NGC 5104 & 9.05 & -0.060 & 0.210 & 2.220 & 0.035 & $1.36 \mathrm{E}+11$ & 32.52 & $2.38 \mathrm{E}-10$ & 67.7 \\
\hline MCG-03-34-064 & 8.99 & 0.022 & 0.488 & 2.204 & 0.042 & $1.19 \mathrm{E}+11$ & {$[27.08]$} & {$[2.27 \mathrm{E}-10]$} & 63.7 \\
\hline NGC 5135 & 9.49 & 0.195 & 0.084 & 1.804 & 0.014 & $1.27 \mathrm{E}+11$ & {$[35.41]$} & {$[2.79 \mathrm{E}-10]$} & 24.4 \\
\hline IC 4280 & 9.49 & 0.391 & 0.109 & 1.661 & 0.019 & $1.45 \mathrm{E}+11$ & 25.24 & $1.74 \mathrm{E}-10$ & 16.7 \\
\hline NGC 5256 & 9.88 & -0.176 & 0.107 & 1.679 & 0.018 & $1.66 \mathrm{E}+11$ & 65.00 & $3.92 \mathrm{E}-10$ & 20.1 \\
\hline Arp 240 & 10.32 & -0.592 & 0.043 & 1.292 & 0.007 & $3.02 \mathrm{E}+11$ & 77.47 & $2.57 \mathrm{E}-10$ & 9.1 \\
\hline UGC 08739 & 9.29 & -0.730 & 0.183 & 1.854 & 0.030 & $9.89 \mathrm{E}+10$ & 24.99 & $2.53 \mathrm{E}-10$ & 34.1 \\
\hline NGC 5331 & 9.75 & 0.011 & 0.029 & 1.911 & 0.005 & $2.66 \mathrm{E}+11$ & 80.41 & $3.02 \mathrm{E}-10$ & 32.7 \\
\hline Arp 84 & 9.08 & 0.398 & 0.118 & 1.993 & 0.021 & $2.11 \mathrm{E}+11$ & 20.99 & $9.94 \mathrm{E}-11$ & 35.7 \\
\hline CGCG 247-020 & 8.48 & -0.627 & 1.038 & 2.911 & 0.167 & $5.54 \mathrm{E}+10$ & 42.81 & $7.72 \mathrm{E}-10$ & 380.3 \\
\hline NGC 5653 & 9.49 & 0.017 & 0.077 & 1.640 & 0.013 & $1.03 \mathrm{E}+11$ & 24.24 & $2.36 \mathrm{E}-10$ & 17.5 \\
\hline IRAS F14348-1447 & 9.92 & -0.792 & 0.138 & 2.466 & 0.023 & $3.74 \mathrm{E}+11$ & 428.54 & $1.14 \mathrm{E}-09$ & 141.6 \\
\hline NGC 5734 & 9.34 & -0.237 & 0.103 & 1.813 & 0.017 & $1.89 \mathrm{E}+11$ & 25.25 & $1.33 \mathrm{E}-10$ & 27.7 \\
\hline VV 340a & 9.80 & -0.376 & 0.026 & 1.950 & 0.046 & $3.15 \mathrm{E}+11$ & 98.31 & $3.12 \mathrm{E}-10$ & 39.3 \\
\hline CGCG 049-057 & 7.20 & 5.900 & 5.898 & 4.156 & 1.072 & $1.97 \mathrm{E}+10$ & 39.16 & $1.99 \mathrm{E}-09$ & 770.7 \\
\hline VV 705 & 9.86 & -0.559 & 0.152 & 2.061 & 0.025 & $1.56 \mathrm{E}+11$ & 147.81 & $9.48 \mathrm{E}-10$ & 52.9 \\
\hline IRAS F15250+3608 & 9.82 & -0.169 & 0.191 & 2.264 & 0.034 & $6.08 \mathrm{E}+10$ & 211.11 & $3.47 \mathrm{E}-09$ & 77.0 \\
\hline NGC 5936 & 9.69 & -0.398 & 0.064 & 1.450 & 0.011 & $9.68 \mathrm{E}+10$ & 25.23 & $2.61 \mathrm{E}-10$ & 12.5 \\
\hline UGC 09913 & 8.85 & 0.997 & 0.329 & 3.423 & 0.038 & $1.16 \mathrm{E}+11$ & 327.74 & 2.82E-09 & 813.3 \\
\hline NGC 5990 & 9.32 & -0.190 & 0.106 & 1.817 & 0.018 & $1.42 \mathrm{E}+11$ & [24.19] & {$[1.70 \mathrm{E}-10]$} & 27.7 \\
\hline NGC 6052 & 10.01 & -0.755 & 0.050 & 1.080 & 0.009 & $4.76 \mathrm{E}+10$ & 24.09 & $5.06 \mathrm{E}-10$ & 5.8 \\
\hline NGC 6090 & 10.26 & -0.176 & 0.017 & 1.325 & 0.003 & $2.24 \mathrm{E}+11$ & 71.10 & $3.17 \mathrm{E}-10$ & 8.9 \\
\hline IRAS F16164-0746 & 9.05 & -0.431 & 0.161 & 2.573 & 0.028 & $7.34 \mathrm{E}+10$ & 72.75 & $9.91 \mathrm{E}-10$ & 167.0 \\
\hline CGCG 052-037 & 8.97 & 0.430 & 0.352 & 2.475 & 0.061 & $1.18 \mathrm{E}+11$ & 48.75 & $4.13 \mathrm{E}-10$ & 107.3 \\
\hline NGC 6240 & 9.59 & 0.003 & 0.168 & 2.336 & 0.031 & $3.90 \mathrm{E}+11$ & [148.44] & [3.81E-10] & 87.1 \\
\hline NGC 6286 & 9.39 & -0.298 & 0.114 & 1.976 & 0.019 & $1.64 \mathrm{E}+11$ & 41.26 & $2.51 \mathrm{E}-10$ & 41.0 \\
\hline IRAS F17132+5313 & 9.53 & 0.364 & 0.073 & 2.435 & 0.013 & $1.72 \mathrm{E}+11$ & 159.67 & $9.28 \mathrm{E}-10$ & 99.8 \\
\hline IRAS F17207-0014 & 7.31 & 4.576 & 8.665 & 5.154 & 1.574 & $1.52 \mathrm{E}+11$ & 501.22 & $3.29 \mathrm{E}-09$ & 12840.8 \\
\hline UGC 11041 & 9.11 & -0.559 & 0.174 & 2.000 & 0.029 & $7.92 \mathrm{E}+10$ & 22.85 & $2.89 \mathrm{E}-10$ & 45.9 \\
\hline CGCG 141-034 & 8.60 & -1.561 & 0.773 & 2.597 & 0.117 & $5.60 \mathrm{E}+10$ & 27.39 & $4.89 \mathrm{E}-10$ & 222.8 \\
\hline NGC 6621 & 9.36 & 0.203 & 0.126 & 1.930 & 0.021 & $1.55 \mathrm{E}+11$ & 34.28 & $2.21 \mathrm{E}-10$ & 32.5 \\
\hline IC 4687 & 9.73 & -0.465 & 0.065 & 1.895 & 0.011 & $1.60 \mathrm{E}+11$ & 74.70 & $4.66 \mathrm{E}-10$ & 35.3 \\
\hline NGC 6670AB & 9.36 & 0.177 & 0.049 & 2.290 & 0.008 & $1.90 \mathrm{E}+11$ & 78.26 & $4.12 \mathrm{E}-10$ & 74.9 \\
\hline IC 4734 & 9.30 & -0.696 & 0.141 & 2.045 & 0.023 & $9.10 \mathrm{E}+10$ & 39.30 & $4.32 \mathrm{E}-10$ & 52.6 \\
\hline ESO 593-IG008 & 9.72 & 1.894 & 0.439 & 2.209 & 0.080 & $3.53 \mathrm{E}+11$ & 150.38 & $4.26 \mathrm{E}-10$ & 42.9 \\
\hline IRAS F19297-0406 & 8.05 & 0.433 & 1.073 & 4.401 & 0.179 & $1.72 \mathrm{E}+11$ & 494.79 & $2.88 \mathrm{E}-09$ & 9041.1 \\
\hline ESO 339-G011 & 8.88 & 0.764 & 0.380 & 2.322 & 0.066 & $1.03 \mathrm{E}+11$ & {$[27.62]$} & [2.69E-10] & 68.9 \\
\hline NGC 6907 & 9.98 & -0.622 & 0.009 & 1.134 & 0.001 & $1.54 \mathrm{E}+11$ & 25.24 & $1.64 \mathrm{E}-10$ & 6.3 \\
\hline NGC 6926 & 9.71 & -0.757 & 0.090 & 1.609 & 0.015 & $1.95 \mathrm{E}+11$ & {$[37.50]$} & [1.92E-10] & 19.5 \\
\hline CGCG 448-020 & 10.28 & -1.134 & 0.099 & 1.662 & 0.016 & $1.34 \mathrm{E}+11$ & 156.77 & $1.17 \mathrm{E}-09$ & 23.9 \\
\hline ESO 286-IG019 & 9.95 & -0.206 & 0.027 & 2.108 & 0.005 & $1.13 \mathrm{E}+11$ & 203.56 & $1.80 \mathrm{E}-09$ & 54.3 \\
\hline ESO 286-G035 & 9.09 & 0.196 & 0.176 & 2.102 & 0.030 & $5.84 \mathrm{E}+10$ & 27.58 & $4.72 \mathrm{E}-10$ & 48.4 \\
\hline ESO 343-IG013 & 9.12 & 0.173 & 0.214 & 2.014 & 0.036 & $6.75 \mathrm{E}+10$ & 24.22 & $3.59 \mathrm{E}-10$ & 39.8 \\
\hline NGC 7130 & 9.71 & 0.327 & 0.017 & 1.712 & 0.003 & $1.45 \mathrm{E}+11$ & [46.88] & [3.23E-10] & 19.1 \\
\hline IC 5179 & 9.62 & -0.137 & 0.058 & 1.619 & 0.010 & $1.23 \mathrm{E}+11$ & 31.01 & $2.52 \mathrm{E}-10$ & 17.3 \\
\hline ESO 602-G025 & 9.30 & -0.169 & 0.264 & 2.037 & 0.044 & $1.37 \mathrm{E}+11$ & 38.38 & $2.80 \mathrm{E}-10$ & 45.6 \\
\hline UGC 12150 & 7.59 & 5.341 & 5.742 & 3.760 & 1.043 & $1.10 \mathrm{E}+11$ & 38.87 & $3.55 \mathrm{E}-10$ & 386.2 \\
\hline IRAS F22491-1808 & 10.00 & 0.176 & 0.076 & 2.198 & 0.013 & $2.27 \mathrm{E}+11$ & 279.16 & $1.23 \mathrm{E}-09$ & 60.7 \\
\hline NGC 7469 & 10.02 & -0.748 & 0.008 & 1.632 & 0.001 & $2.39 \mathrm{E}+11$ & {$[80.26]$} & [3.35E-10] & 20.6 \\
\hline CGCG 453-062 & 8.61 & 1.285 & 0.659 & 2.768 & 0.116 & $8.99 \mathrm{E}+10$ & 41.52 & $4.62 \mathrm{E}-10$ & 165.3 \\
\hline ESO 148-IG002 & 10.01 & -0.282 & 0.025 & 2.054 & 0.004 & $1.06 \mathrm{E}+11$ & 204.60 & $1.94 \mathrm{E}-09$ & 48.8 \\
\hline IC 5298 & 9.15 & -0.553 & 0.312 & 2.451 & 0.051 & $1.29 \mathrm{E}+11$ & 69.67 & $5.38 \mathrm{E}-10$ & 129.7 \\
\hline NGC 7552 & 9.32 & 0.403 & 0.005 & 1.793 & 0.001 & $7.62 \mathrm{E}+10$ & {$[22.98]$} & [3.01E-10] & 22.5 \\
\hline NGC 7592 & 10.07 & -1.240 & 0.067 & 1.332 & 0.011 & $1.13 \mathrm{E}+11$ & [47.03] & {$[4.15 \mathrm{E}-10]$} & 11.4 \\
\hline ESO 077-IG014 & 8.90 & 1.576 & 0.211 & 2.856 & 0.038 & $1.77 \mathrm{E}+11$ & 100.48 & $5.68 \mathrm{E}-10$ & 185.3 \\
\hline NGC 7674 & 10.00 & -0.489 & 0.022 & 1.560 & 0.004 & $3.03 \mathrm{E}+11$ & {$[65.76]$} & [2.17E-10] & 16.4 \\
\hline IRAS F23365+3604 & 9.33 & 3.515 & 0.183 & 2.872 & 0.033 & $1.41 \mathrm{E}+11$ & 276.72 & $1.96 \mathrm{E}-09$ & 99.8 \\
\hline MCG-01-60-022 & 9.52 & -0.671 & 0.031 & 1.752 & 0.005 & $2.08 \mathrm{E}+11$ & 33.35 & $1.61 \mathrm{E}-10$ & 26.6 \\
\hline Arp 86 & 9.40 & -0.263 & 0.030 & 1.667 & 0.005 & $2.84 \mathrm{E}+11$ & 21.21 & $7.47 \mathrm{E}-11$ & 19.9 \\
\hline
\end{tabular}


Table 3

(Continued)

\begin{tabular}{|c|c|c|c|c|c|c|c|c|c|}
\hline $\begin{array}{c}\text { System } \\
\text { (1) }\end{array}$ & $\begin{array}{c}\log \left(L_{\mathrm{FUV}}\right) \\
(2)\end{array}$ & $\begin{array}{c}\beta(G A L E X) \\
(3)\end{array}$ & $\begin{array}{c}\sigma_{\beta(G A L E X)} \\
(4)\end{array}$ & $\begin{array}{l}\text { IRX } \\
(5) \\
\end{array}$ & $\begin{array}{c}\sigma_{\mathrm{IRX}} \\
(6) \\
\end{array}$ & $\begin{array}{c}\text { Mass } \\
\left(M_{\odot}\right) \\
(7) \\
\end{array}$ & $\begin{array}{c}\mathrm{SFR} \\
\left(M_{\odot} \mathrm{yr}^{-1}\right) \\
(8)\end{array}$ & $\begin{array}{c}\mathrm{SSFR} \\
\left(\mathrm{yr}^{-1}\right) \\
(9)\end{array}$ & $\begin{array}{l}\text { IR/UV } \\
(10) \\
\end{array}$ \\
\hline NGC 7771 & 9.78 & -0.880 & 0.006 & 1.621 & 0.001 & $4.00 \mathrm{E}+11$ & 45.12 & $1.13 \mathrm{E}-10$ & 20.6 \\
\hline
\end{tabular}

Notes. Column 1: system name; Column 2: the total FUV luminosity in $\log _{10}$ Solar units; Column 3: $\beta(G A L E X)$ calculated as described in Section 3.1, Equation (1); Column 4: $\beta(G A L E X)$ uncertainty; Column 5: ratio of IR to FUV flux IRX calculated as described in Section 3.1; Column 6: IRX uncertainty; Column 7: stellar mass calculated as described in Section 3.2; Column 8: star formation rate calculated as described in Section 3.2. Values in brackets should be considered upper limits due to possible AGN contamination. Column 9: specific star formation rate calculated as described in Section 3.2. Values in brackets should be considered upper limits due to possible AGN contamination. Column 10: IR/UV ratio calculated as described in Section 3.2, Equation (2).

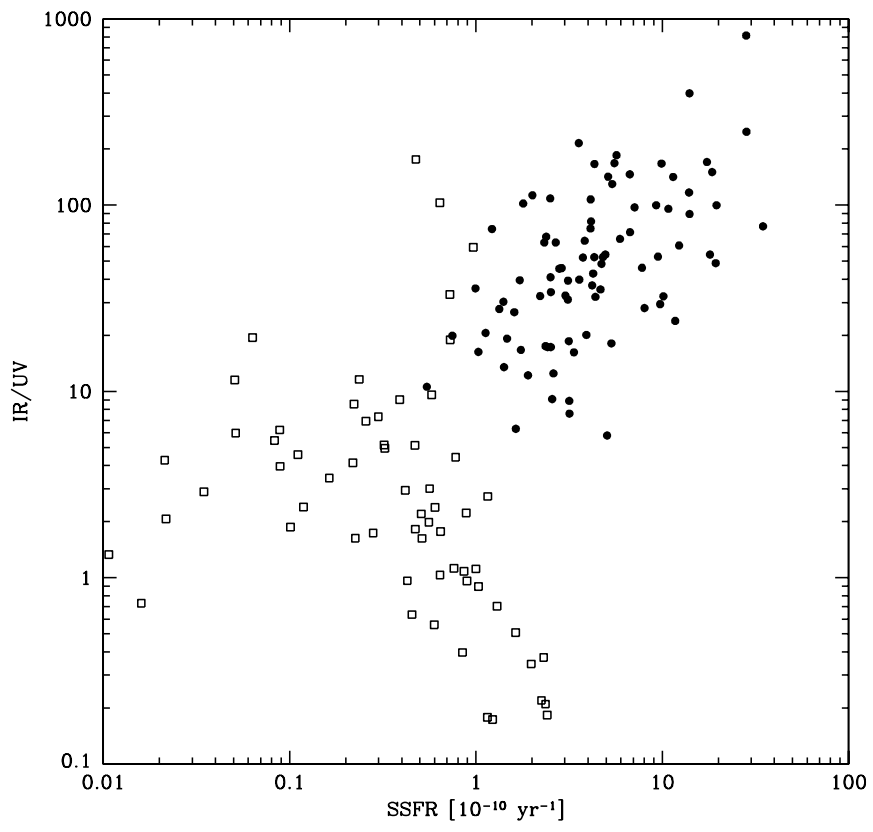

Figure 3. IR/UV ratio plotted against specific star formation rate. Solid circles are GOALS galaxies (not including those with IRAC colors suggesting a strong AGN), while open squares are SINGS galaxies (Dale et al. 2007). The GOALS outlier at the high IR/UV, high SSFR extreme is Arp 220. LIRGs and ULIRGs have much higher IR/UV ratios and SSFR than lower luminosity galaxies, and the two quantities are correlated for GOALS systems and anti-correlated for SINGS galaxies with SSFR $>10^{-11} \mathrm{yr}^{-1}$.

together, the GOALS and SINGS sources span nearly 4 orders of magnitude in IR/UV at high SSFR $\left(>10^{-10} \mathrm{yr}^{-1}\right)$, probing very different star-forming populations.

To investigate subpopulations of the GOALS sample in SSFR, we define bins with SSFR $<3 \times 10^{-10} \mathrm{yr}^{-1}, 3 \times 10^{-10}<$ SSFR $<6 \times 10^{-10} \mathrm{yr}^{-1}$, and SSFR $>6 \times 10^{-10} \mathrm{yr}^{-1}$ : galaxies which span the same range of SSFR as the SINGS sample, galaxies with up to twice the SSFR as the most extreme SINGS galaxies, and galaxies with more than twice the SSFR of the most extreme SINGS galaxies, respectively. These subpopulations are plotted on the IRX- $\beta(G A L E X)$ diagram in Figure 4 . The systems with higher SSFR have higher median offsets from the starburst relation than systems with lower SSFR. Median $\beta$ values are $-0.2 \pm 0.2,0.1 \pm 0.1$, and $-0.20 \pm 0.09$ (high, medium, and low SSFR bins, respectively). Median IRX values are $2.35 \pm 0.09,2.10 \pm 0.08$, and $1.81 \pm 0.06$, respectively. Systems with $\beta<0.5$ allowing a direct comparison to the starburst relation have median vertical deviations of $0.9 \pm 0.1$, $0.4 \pm 0.1$, and $0.37 \pm 0.09$, respectively.

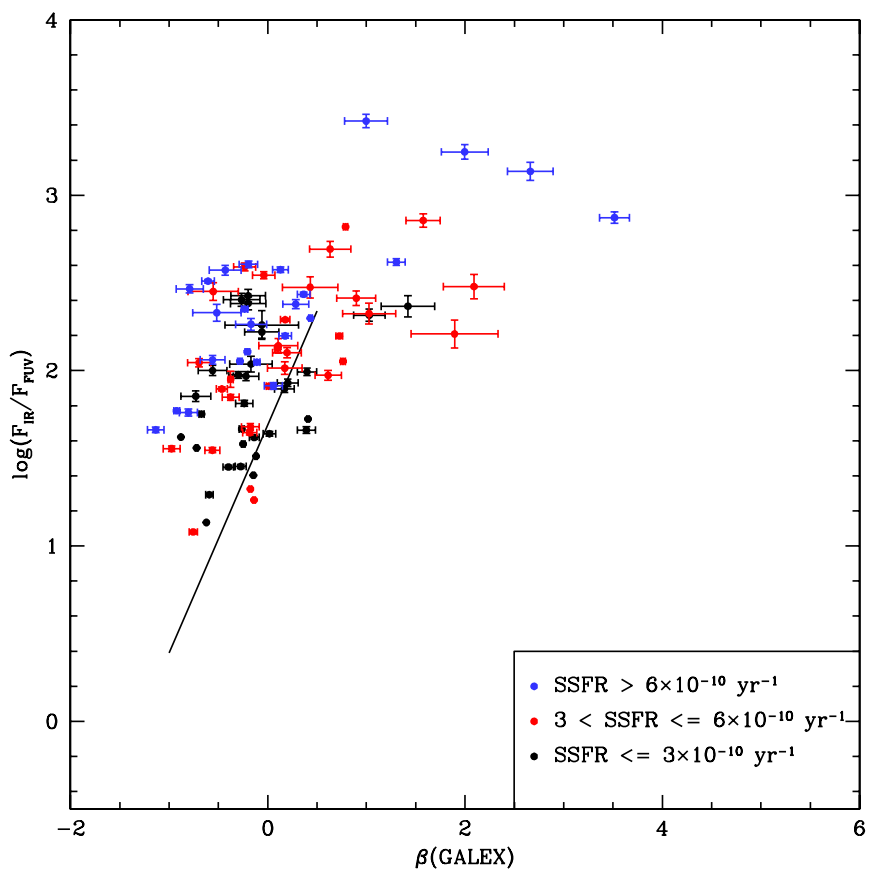

Figure 4. IRX $-\beta(G A L E X)$ plot with GOALS systems (not including those with IRAC colors suggesting a strong AGN) color coded by specific SFR: black points have SSFR within the range spanned by SINGS galaxies, red points have up to twice the SSFR of any SINGS galaxy, and blue points have more than twice the SSFR of any SINGS galaxy. The solid line is the same as in Figure 1. Systems with higher SSFR are systematically redder in $\beta$ and have larger IRX than systems with lower SSFR.

\subsection{Resolved Systems}

A number of the interacting LIRGs in GOALS are near enough to resolve with both GALEX and Spitzer and derive IR and UV properties for each galaxy. Derived quantities for the galaxies in resolved systems are presented in Table 4. The component galaxies of resolved pair/triple systems are plotted on the IRX- $\beta(G A L E X)$ diagram in Figure 5. Many GOALS systems consist of an LIRG with one or more sub-LIRG companions. The sub-LIRG galaxies are on average consistent with the GDP sample. LIRGs are on average offset above the starburst relation, with $L_{\mathrm{IR}}>10^{11.4} L_{\odot}$ systems having larger offsets than lower luminosity LIRGs. For systems with $\beta<0.5$, median offsets are $1.1 \pm 0.2$ and $0.4 \pm 0.1$ for the $L_{\mathrm{IR}}>10^{11.4} L_{\odot}$ and $10^{11} L_{\odot}<L_{\mathrm{IR}}<10^{11.4} L_{\odot}$ populations, respectively. An individual galaxy in general does not lie in the same region of the IRX $-\beta$ diagram as the LIRG system of which it is a component; see Section 4.3 for further discussion.

Of the 18 resolved systems for which masses could be estimated, the median mass ratio of the galaxy companions is 
Table 4

Derived Quantities_-Resolved Components

\begin{tabular}{|c|c|c|c|c|c|c|c|c|}
\hline $\begin{array}{c}\text { Galaxy } \\
\text { (1) }\end{array}$ & $\begin{array}{c}\log \left(L_{\mathrm{FUV}}\right) \\
(2)\end{array}$ & $\begin{array}{c}\beta(G A L E X) \\
\text { (3) }\end{array}$ & $\begin{array}{c}\sigma_{\beta(G A L E X)} \\
(4)\end{array}$ & $\begin{array}{l}\text { IRX } \\
(5) \\
\end{array}$ & $\begin{array}{c}\sigma_{\text {IRX }} \\
(6)\end{array}$ & $\begin{array}{c}\text { Mass } \\
\left(M_{\odot}\right) \\
(7)\end{array}$ & $\begin{array}{c}\mathrm{SFR} \\
\left(M_{\odot} \mathrm{yr}^{-1}\right) \\
(8)\end{array}$ & $\begin{array}{c}\text { SSFR } \\
\left(\mathrm{yr}^{-1}\right) \\
(9)\end{array}$ \\
\hline NGC 0034 & 9.69 & -0.91 & 0.15 & 1.739 & 0.013 & $8.92 \mathrm{E}+10$ & {$[46.91]$} & {$[5.26 \mathrm{E}-10]$} \\
\hline NGC 0035 & 9.35 & 0.53 & 0.19 & 1.257 & 0.020 & $2.05 \mathrm{E}+10$ & 6.99 & $3.41 \mathrm{E}-10$ \\
\hline MCG-02-01-052 & 9.89 & -0.39 & 0.03 & 0.468 & 0.004 & $\ldots$ & 3.95 & $\ldots$ \\
\hline MCG-02-01-051 & 9.95 & -0.64 & 0.03 & 1.501 & 0.003 & $\ldots$ & 48.63 & $\ldots$ \\
\hline NGC 0232 & 9.04 & -0.26 & 0.55 & 2.312 & 0.037 & $1.10 \mathrm{E}+11$ & 39.14 & $3.57 \mathrm{E}-10$ \\
\hline NGC 0235A & 8.73 & -0.18 & 1.05 & 1.998 & 0.062 & $8.46 \mathrm{E}+10$ & {$[9.21]$} & [1.09E-10] \\
\hline NGC 0317A & 7.84 & 4.09 & 0.34 & 1.120 & 0.060 & $8.15 \mathrm{E}+10$ & 0.16 & $1.95 \mathrm{E}-12$ \\
\hline NGC 0317B & 8.77 & 0.76 & 0.34 & 2.412 & 0.060 & $3.44 \mathrm{E}+10$ & 26.63 & $7.73 \mathrm{E}-10$ \\
\hline IC $1623 A$ & 10.31 & -0.71 & 0.01 & 0.773 & 0.002 & $1.37 \mathrm{E}+11$ & 21.07 & $1.54 \mathrm{E}-10$ \\
\hline IC $1623 B$ & 8.88 & 0.65 & 0.01 & 2.703 & 0.002 & $4.72 \mathrm{E}+10$ & [67.19] & [1.42E-09] \\
\hline ESO 244-G012 NED01 & 9.45 & -0.62 & 0.10 & 0.042 & 0.017 & $\ldots$ & 0.53 & $\ldots$ \\
\hline ESO 244-G012 NED02 & 8.79 & 0.49 & 0.10 & 2.584 & 0.017 & $\ldots$ & 41.19 & $\ldots$ \\
\hline UGC 01385 & 8.75 & -0.28 & 0.48 & 2.271 & 0.040 & $4.74 \mathrm{E}+10$ & 18.30 & $3.86 \mathrm{E}-10$ \\
\hline KUG 0152+366 & 9.42 & -0.62 & 0.16 & 0.399 & 0.016 & $1.31 \mathrm{E}+10$ & 1.14 & $8.71 \mathrm{E}-11$ \\
\hline NGC 0876 & 9.59 & -0.12 & 0.02 & 0.923 & 0.002 & $2.43 \mathrm{E}+10$ & 5.60 & $2.31 \mathrm{E}-10$ \\
\hline NGC 0877 & 8.24 & 0.06 & 0.32 & 2.727 & 0.021 & $1.37 \mathrm{E}+11$ & 16.17 & $1.18 \mathrm{E}-10$ \\
\hline MCG+05-06-035 & 9.17 & 1.09 & 0.19 & 1.980 & 0.034 & $1.89 \mathrm{E}+11$ & 24.84 & $1.31 \mathrm{E}-10$ \\
\hline MCG+05-06-036 & 8.80 & 0.88 & 0.19 & 2.672 & 0.034 & $9.86 \mathrm{E}+10$ & 51.76 & $5.25 \mathrm{E}-10$ \\
\hline UGC 02894 & 8.80 & -0.34 & 1.31 & 1.827 & 0.093 & $4.73 \mathrm{E}+10$ & 7.28 & $1.54 \mathrm{E}-10$ \\
\hline CGCG 465-012 & 8.15 & 3.75 & 2.38 & 2.920 & 0.395 & $6.95 \mathrm{E}+10$ & 20.18 & $2.90 \mathrm{E}-10$ \\
\hline ESO 550-IG025 NED01 & 9.28 & -0.01 & 0.24 & 1.994 & 0.041 & $\ldots$ & 32.28 & $\ldots$ \\
\hline ESO 550-IG025 NED02 & 8.64 & 0.55 & 0.24 & 2.497 & 0.041 & $\ldots$ & 23.54 & $\ldots$ \\
\hline CGCG 468-002 NED01 & 7.07 & 4.40 & 1.61 & 3.669 & 0.269 & $6.54 \mathrm{E}+10$ & 9.57 & $1.46 \mathrm{E}-10$ \\
\hline CGCG 468-002 NED02 & 8.30 & -2.57 & 1.61 & 2.751 & 0.269 & $1.83 \mathrm{E}+10$ & {$[19.52]$} & {$[1.06 \mathrm{E}-09]$} \\
\hline IRAS $05083+2441$ & 7.95 & 1.10 & 3.94 & 3.264 & 0.462 & $5.56 \mathrm{E}+10$ & 28.38 & $5.11 \mathrm{E}-10$ \\
\hline 2MASX J05112888+2445593 & 5.66 & 7.79 & 483.06 & 4.650 & 87.595 & $2.30 \mathrm{E}+10$ & 3.54 & $1.54 \mathrm{E}-10$ \\
\hline UGC 03405 & 8.72 & -0.20 & 0.46 & 1.626 & 0.037 & $3.73 \mathrm{E}+10$ & 3.83 & $1.03 \mathrm{E}-10$ \\
\hline UGC 03410 & 8.23 & 0.19 & 1.18 & 2.787 & 0.096 & $8.55 \mathrm{E}+10$ & 18.05 & $2.11 \mathrm{E}-10$ \\
\hline NGC 2341 & 9.15 & 0.80 & 0.31 & 1.830 & 0.032 & $7.10 \mathrm{E}+10$ & 16.69 & $2.35 \mathrm{E}-10$ \\
\hline NGC 2342 & 9.86 & -0.27 & 0.11 & 1.178 & 0.011 & $1.27 \mathrm{E}+11$ & 18.93 & $1.50 \mathrm{E}-10$ \\
\hline NGC 2385 & 7.76 & 4.19 & 0.48 & 1.923 & 0.079 & $2.76 \mathrm{E}+10$ & 0.84 & $3.04 \mathrm{E}-11$ \\
\hline NGC 2388 & 9.72 & -0.72 & 0.03 & 1.453 & 0.002 & $7.73 \mathrm{E}+10$ & 26.11 & $3.38 \mathrm{E}-10$ \\
\hline NGC 2389 & 7.11 & 3.86 & 2.12 & 3.453 & 0.334 & $3.83 \mathrm{E}+10$ & 6.34 & $1.65 \mathrm{E}-10$ \\
\hline NGC 2416 & 9.45 & -0.56 & 0.20 & $\ldots$ & $\ldots$ & $3.30 \mathrm{E}+10$ & $\ldots$ & $\ldots$ \\
\hline $\mathrm{MCG}+02-20-003$ & 9.14 & 0.73 & 0.25 & $\ldots$ & $\ldots$ & $4.92 \mathrm{E}+10$ & $\ldots$ & $\ldots$ \\
\hline 2MASX J09133644-1019296 & 7.90 & 2.62 & 1.02 & 4.049 & 0.181 & $\ldots$ & 154.77 & $\ldots$ \\
\hline 2MASX J09133888-1019196 & 9.45 & 1.10 & 0.06 & 1.942 & 0.010 & $\ldots$ & 42.94 & $\ldots$ \\
\hline UGC 04881 NED02 & 7.98 & 0.82 & 0.22 & 3.285 & 0.038 & $8.52 \mathrm{E}+10$ & 32.23 & $3.78 \mathrm{E}-10$ \\
\hline UGC 04881 NED01 & 8.37 & 0.78 & 0.13 & 3.192 & 0.023 & $1.73 \mathrm{E}+11$ & 63.94 & $3.69 \mathrm{E}-10$ \\
\hline CGCG 239-011 NED01 & 9.63 & -1.39 & 0.23 & 0.345 & 0.020 & $6.25 \mathrm{E}+09$ & 1.65 & $2.64 \mathrm{E}-10$ \\
\hline MCG+08-18-013 & 9.25 & -0.19 & 0.32 & 2.066 & 0.032 & $6.79 \mathrm{E}+10$ & 36.24 & $5.34 \mathrm{E}-10$ \\
\hline 2MASX J11210825-0259399 & 8.26 & -0.51 & 2.74 & 2.127 & 0.154 & $9.64 \mathrm{E}+09$ & 4.23 & $4.39 \mathrm{E}-10$ \\
\hline CGCG 011-076 & 8.25 & 2.09 & 1.27 & 3.134 & 0.157 & $1.21 \mathrm{E}+11$ & 42.19 & $3.48 \mathrm{E}-10$ \\
\hline IC $2810 \mathrm{~A}$ & 9.19 & 0.93 & 0.40 & 2.266 & 0.044 & $1.21 \mathrm{E}+11$ & 49.68 & $4.09 \mathrm{E}-10$ \\
\hline IC $2810 B$ & 8.14 & 0.56 & 3.82 & 3.035 & 0.253 & $6.26 \mathrm{E}+10$ & 26.21 & 4.19E-10 \\
\hline NGC 3690 & 10.00 & -0.87 & 0.03 & 1.766 & 0.005 & $1.26 \mathrm{E}+11$ & [101.44] & {$[8.02 \mathrm{E}-10]$} \\
\hline IC 0694 & 9.63 & -1.06 & 0.03 & 1.780 & 0.005 & $7.79 \mathrm{E}+10$ & [45.19] & {$[5.80 \mathrm{E}-10]$} \\
\hline ESO 440-IG058 NED01 & 8.97 & 0.95 & 0.34 & 1.574 & 0.059 & $\ldots$ & 6.02 & $\ldots$ \\
\hline ESO 440-IG058 NED02 & 8.56 & 1.20 & 0.34 & 2.819 & 0.059 & $\ldots$ & 41.15 & $\ldots$ \\
\hline ESO 267-G029 & 9.39 & -0.70 & 0.52 & 1.537 & 0.037 & $9.56 \mathrm{E}+10$ & 14.52 & $1.52 \mathrm{E}-10$ \\
\hline ESO 267-G030 & 8.99 & -0.06 & 1.00 & 1.986 & 0.082 & $1.61 \mathrm{E}+11$ & 16.52 & $1.03 \mathrm{E}-10$ \\
\hline NGC 4922 NED01 & 8.30 & 2.02 & 0.08 & 0.576 & 0.014 & $\ldots$ & 0.13 & $\ldots$ \\
\hline NGC 4922 NED02 & 8.76 & 0.64 & 0.08 & 2.615 & 0.014 & $\ldots$ & [41.61] & $\ldots$ \\
\hline MCG-02-33-098 & 8.60 & 2.00 & 0.16 & 2.406 & 0.028 & $\ldots$ & 17.52 & $\ldots$ \\
\hline MCG-02-33-099 & 9.07 & -0.13 & 0.16 & 1.593 & 0.028 & $\ldots$ & 7.93 & $\ldots$ \\
\hline VV 250b & 9.42 & -0.10 & 0.11 & $\ldots$ & $\ldots$ & $4.60 \mathrm{E}+10$ & $\ldots$ & $\ldots$ \\
\hline VV 250a & 9.72 & 0.13 & 0.11 & $\ldots$ & $\ldots$ & $6.62 \mathrm{E}+10$ & $\ldots$ & $\ldots$ \\
\hline MRK 266B & 9.51 & -0.22 & 0.11 & 1.853 & 0.018 & $\ldots$ & 40.44 & $\ldots$ \\
\hline MRK 266A & 9.64 & -0.15 & 0.11 & 1.477 & 0.018 & $\ldots$ & 22.48 & $\ldots$ \\
\hline NGC 5257 & 9.77 & -0.33 & 0.13 & 1.541 & 0.014 & $1.42 \mathrm{E}+11$ & 35.66 & $2.50 \mathrm{E}-10$ \\
\hline NGC 5258 & 10.18 & -0.70 & 0.08 & 1.136 & 0.009 & $1.59 \mathrm{E}+11$ & 36.03 & $2.26 \mathrm{E}-10$ \\
\hline NGC 5331 NED01 & 9.15 & 0.45 & 0.03 & 2.393 & 0.005 & $\ldots$ & 60.78 & $\ldots$ \\
\hline NGC 5331 NED02 & 9.62 & -0.16 & 0.03 & 1.398 & 0.005 & $\cdots$ & 18.10 & $\cdots$ \\
\hline NGC 5734 & 8.73 & 1.01 & 0.48 & 2.204 & 0.051 & $1.23 \mathrm{E}+11$ & 15.09 & $1.23 \mathrm{E}-10$ \\
\hline
\end{tabular}


Table 4

(Continued)

\begin{tabular}{|c|c|c|c|c|c|c|c|c|}
\hline $\begin{array}{c}\text { Galaxy } \\
\text { (1) }\end{array}$ & $\begin{array}{c}\log \left(L_{\mathrm{FUV}}\right) \\
(2)\end{array}$ & $\beta(G A L E X)$ & $\begin{array}{c}\sigma_{\beta(G A L E X)} \\
(4)\end{array}$ & $\begin{array}{l}\text { IRX } \\
(5) \\
\end{array}$ & $\begin{array}{c}\sigma_{\mathrm{IRX}} \\
(6) \\
\end{array}$ & $\begin{array}{c}\text { Mass } \\
\left(M_{\odot}\right) \\
(7) \\
\end{array}$ & $\begin{array}{c}\mathrm{SFR} \\
\left(M_{\odot} \mathrm{yr}^{-1}\right) \\
(8)\end{array}$ & $\begin{array}{c}\mathrm{SSFR} \\
\left(\mathrm{yr}^{-1}\right) \\
(9) \\
\end{array}$ \\
\hline NGC 5743 & 9.34 & -0.24 & 0.20 & 1.401 & 0.017 & $6.63 \mathrm{E}+10$ & 9.56 & $1.44 \mathrm{E}-10$ \\
\hline VV 340b & 9.58 & -0.39 & 0.03 & 1.425 & 0.005 & $6.86 \mathrm{E}+10$ & 17.56 & $2.56 \mathrm{E}-10$ \\
\hline VV 340a & 9.05 & -0.44 & 0.06 & 2.610 & 0.010 & $2.46 \mathrm{E}+11$ & 79.04 & $3.21 \mathrm{E}-10$ \\
\hline NGC 6285 & 9.11 & -0.22 & 0.25 & 1.532 & 0.026 & $3.84 \mathrm{E}+10$ & 7.58 & $1.97 \mathrm{E}-10$ \\
\hline NGC 6286 & 9.07 & -0.39 & 0.28 & 2.204 & 0.027 & $1.26 \mathrm{E}+11$ & 33.01 & $2.63 \mathrm{E}-10$ \\
\hline NGC 6621 & 8.87 & 1.09 & 0.13 & 2.401 & 0.021 & $\ldots$ & 32.78 & $\ldots$ \\
\hline NGC 6621 SE & 9.17 & -0.69 & 0.13 & 0.355 & 0.021 & $\ldots$ & 0.58 & $\ldots$ \\
\hline NGC 6622 & 7.78 & 2.81 & 0.13 & 1.451 & 0.021 & $\ldots$ & 0.29 & $\ldots$ \\
\hline IC 4686 & 9.54 & -0.12 & 0.07 & 1.499 & 0.012 & $2.28 \mathrm{E}+10$ & 19.22 & $8.41 \mathrm{E}-10$ \\
\hline IC 4687 & 9.10 & -0.38 & 0.07 & 2.241 & 0.012 & $8.69 \mathrm{E}+10$ & 38.51 & $4.43 \mathrm{E}-10$ \\
\hline IC 4689 & 8.77 & -0.57 & 0.62 & 2.176 & 0.045 & $5.04 \mathrm{E}+10$ & 15.49 & $3.07 \mathrm{E}-10$ \\
\hline NGC 6670B & 8.77 & 0.27 & 0.08 & 2.558 & 0.014 & $1.25 \mathrm{E}+11$ & 36.78 & $2.95 \mathrm{E}-10$ \\
\hline NGC 6670A & 9.14 & -0.17 & 0.05 & 2.229 & 0.008 & $6.53 \mathrm{E}+10$ & 41.39 & $6.34 \mathrm{E}-10$ \\
\hline ESO 343-IG013 NED01 & 8.95 & 0.12 & 0.21 & 1.649 & 0.036 & $\ldots$ & 6.85 & $\ldots$ \\
\hline ESO 343-IG013 NED02 & 8.65 & 0.28 & 0.21 & 2.342 & 0.036 & $\ldots$ & 17.01 & $\ldots$ \\
\hline NGC 7469 & 10.00 & -0.78 & 0.01 & 1.585 & 0.001 & $1.95 \mathrm{E}+11$ & [66.69] & [3.41E-10] \\
\hline IC 5283 & 8.61 & -0.02 & 0.08 & 2.179 & 0.007 & $4.39 \mathrm{E}+10$ & 10.72 & $2.44 \mathrm{E}-10$ \\
\hline NGC 7592A & 9.08 & -0.83 & 0.07 & 2.097 & 0.011 & $\ldots$ & {$[25.85]$} & $\ldots$ \\
\hline NGC 7592B & 10.02 & -1.29 & 0.07 & 0.991 & 0.011 & $\ldots$ & 17.96 & $\ldots$ \\
\hline ESO 077-IG014 NED01 & 8.88 & 1.21 & 0.21 & 2.448 & 0.038 & $1.23 \mathrm{E}+11$ & 37.28 & $3.04 \mathrm{E}-10$ \\
\hline ESO 077-IG014 NED02 & 7.60 & 4.73 & 0.21 & 3.960 & 0.038 & $5.42 \mathrm{E}+10$ & 62.98 & $1.16 \mathrm{E}-09$ \\
\hline NGC 7674 & 9.96 & -0.64 & 0.02 & 1.587 & 0.004 & $2.49 \mathrm{E}+11$ & [61.26] & [2.46E-10] \\
\hline NGC 7674A & 8.94 & 0.69 & 0.02 & 1.072 & 0.004 & $5.37 \mathrm{E}+10$ & 1.77 & $3.29 \mathrm{E}-11$ \\
\hline MRK 933 & 9.14 & -0.11 & 0.09 & 0.772 & 0.008 & $6.43 \mathrm{E}+09$ & 1.41 & $2.19 \mathrm{E}-10$ \\
\hline MCG-01-60-021 & 8.21 & $\ldots$ & $\ldots$ & 1.963 & 0.035 & $1.30 \mathrm{E}+11$ & 2.59 & $2.00 \mathrm{E}-11$ \\
\hline MCG-01-60-022 & 9.56 & -0.61 & 0.07 & 1.654 & 0.005 & $7.16 \mathrm{E}+10$ & 28.44 & $3.97 \mathrm{E}-10$ \\
\hline NGC 7769 & 9.78 & -0.88 & 0.01 & 0.961 & 0.001 & $1.38 \mathrm{E}+11$ & 9.53 & $6.90 \mathrm{E}-11$ \\
\hline NGC 7770 & 9.28 & -0.80 & 0.01 & 1.396 & 0.002 & $2.59 \mathrm{E}+10$ & {$[8.16]$} & [3.15E-10] \\
\hline NGC 7771 & 8.96 & 0.52 & 0.02 & 2.214 & 0.003 & $2.37 \mathrm{E}+11$ & 25.79 & $1.09 \mathrm{E}-10$ \\
\hline
\end{tabular}

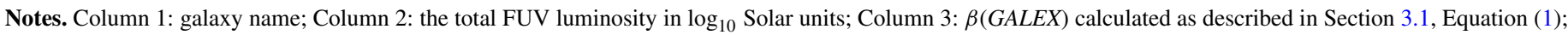

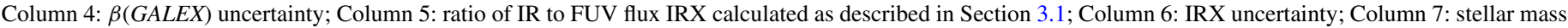

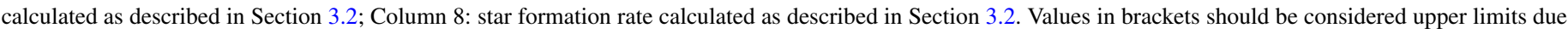

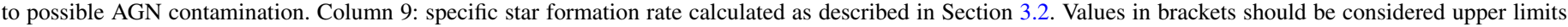
due to possible AGN contamination.

2.6:1, with a range from 1.1:1 to 8.1:1. The high mass component of these pairs/triples is, on average, offset above the starburst relation (Figure 6), while the low mass components are, on average, consistent with the starburst relation. For systems with $\beta<0.5$, median offsets are $0.9 \pm 0.1$ and $0.3 \pm 0.1$ for the high mass and low mass components, respectively.

\section{DISCUSSION}

The complete sample of the nearest LIRGs and ULIRGs that comprise GOALS is ideal for studying the relationship between the IR and UV properties of LIRGs. A key diagnostic tool which we explore in this paper is the IRX $-\beta(G A L E X)$ diagram, comparing the IR excess (ratio of IR to FUV emission) to the FUV-NUV color parameterized as the power-law slope $\beta(G A L E X)$. If a class of galaxies, such as starburst galaxies, follows tight relations on this diagram, then the measurement of the rest-frame UV color allows IRX and thus $L_{\mathrm{IR}}$ to be derived. This is of particular interest at high redshift, where $L_{\mathrm{IR}}$ can only be directly measured using far-infrared and submillimeter observations but rest-frame UV observations can be made at visual wavelengths in deep surveys. Since LIRGs contribute significantly to the star formation activity at high redshift (e.g., Magnelli et al. 2009), understanding the IRX $-\beta(G A L E X)$ relation in this population is extremely important. The IRX $-\beta(G A L E X)$ diagram, and the combination of UV and IR data more generally, provides an indication of the obscuration to the young stars (or active nucleus) within a galaxy. This can provide a rough test of the evolutionary sequence in which some starburst galaxies transition from LIRGs to ULIRGs to QSO hosts over the course of a major merger event as the dust and gas is first funneled toward the nuclei fueling a starburst, only to be cleared away by the action of AGN and starburst winds in the final stages of the transformation to a QSO.

To estimate the importance of high- $\beta$ galaxies among the IR population as a whole, the fraction of the total IR luminosity integrated over all 629 galaxies in the RBGS contributed by the 112 LIRGs and ULIRGs of the GOALS GALEX sample is shown as a function of $\beta$ in Figure 7. Within the GOALS sample, more luminous systems have, on average, larger IRX and redder $\beta$ values than less luminous systems while maintaining roughly the same offset from the starburst relation. As shown in Figure 7, a minimum of $19 \%$ of the total infrared luminosity of the 629 galaxies that comprise the RBGS is produced in LIRGs and ULIRGs with a $\beta>0$ (IUE or GALEX). These red sources are typically absent from UV-selected samples at high redshift, 


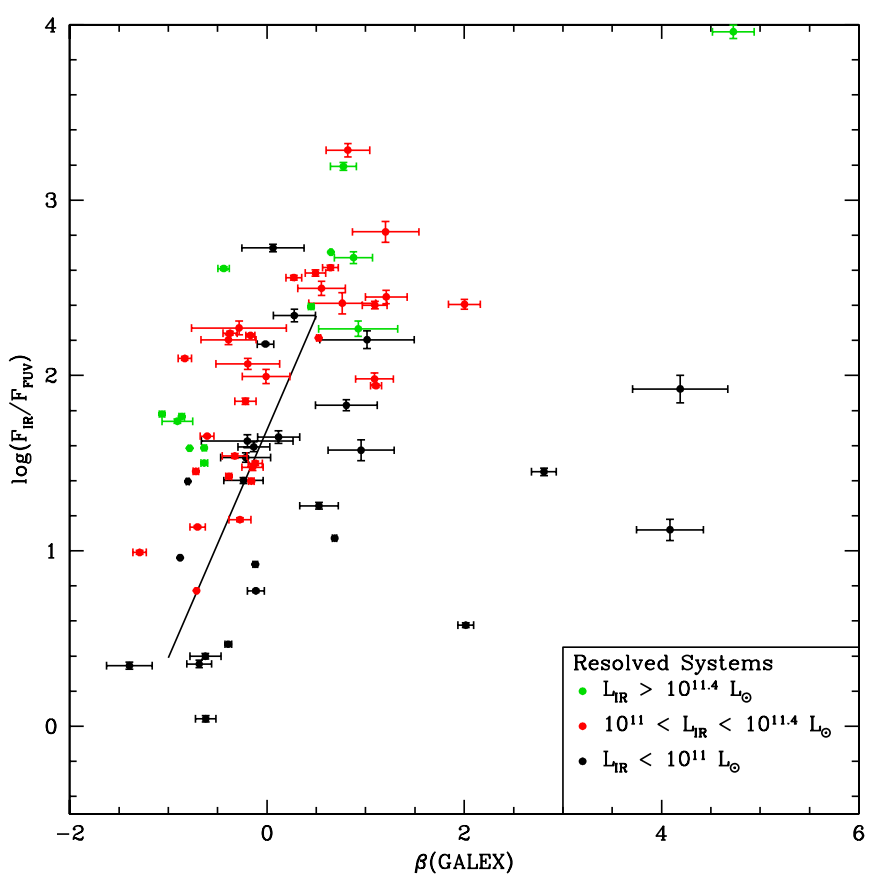

Figure 5. IRX $-\beta(G A L E X)$ plot showing the locations of individual galaxies in resolved pairs. As in Figure 1 , black points have $L_{\mathrm{IR}}<10^{11} L_{\odot}$, red points have $10^{11} L_{\odot}<L_{\mathrm{IR}}<10^{11.4} L_{\odot}$, and green points have $10^{11.4} L_{\odot}<L_{\mathrm{IR}}<10^{11.8} L_{\odot}$. The solid line is the same as in Figure 1. Sub-LIRG galaxies are on average consistent with the GDP sample. LIRGs are on average offset above the starburst relation, with $L_{\mathrm{IR}}>10^{11.4} L_{\odot}$ systems having larger offsets than lower luminosity LIRGs.

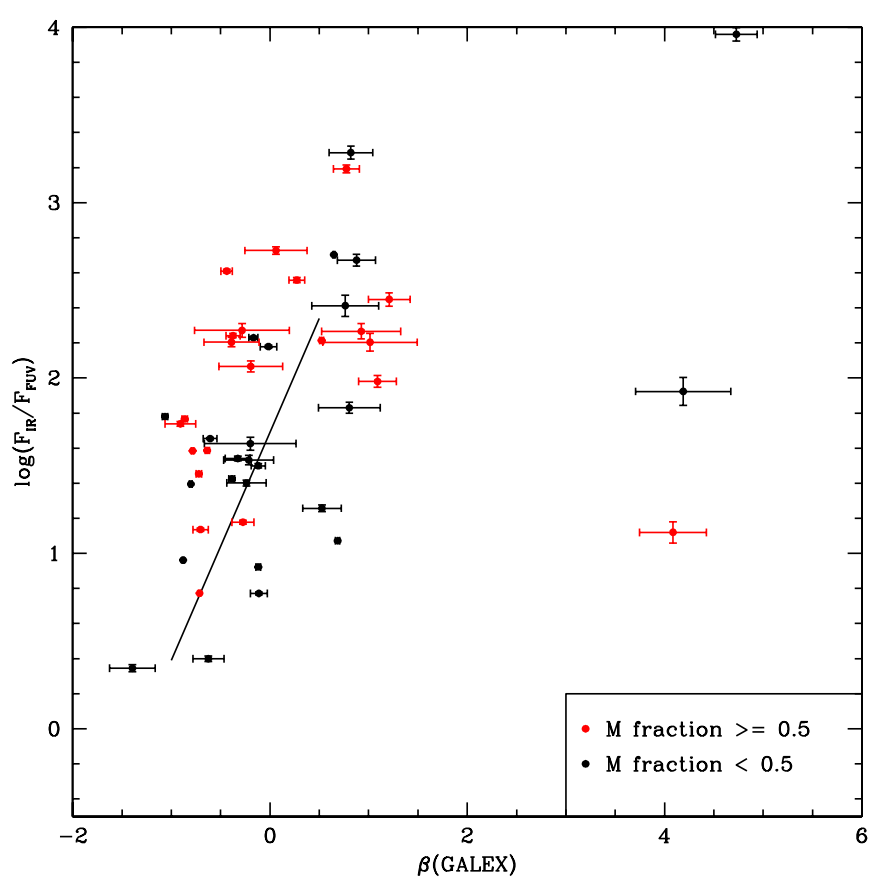

Figure 6. IRX $-\beta(G A L E X)$ plot showing the location of individual galaxies in close pairs for which a mass could be estimated. The galaxy with $>50 \%$ of the mass in each system is shown in red, while other galaxies are in black. The solid line shows the starburst relation, as in Figure 1. On average, the high mass galaxy in a system is offset above the starburst relation, while the lower mass galaxy lies slightly below the starburst relation.

regardless of their estimated IR luminosity. This is a strict lower limit, since there are a number of low- $z$ LIRGs not observed or detected with GALEX which might have large $\beta$.

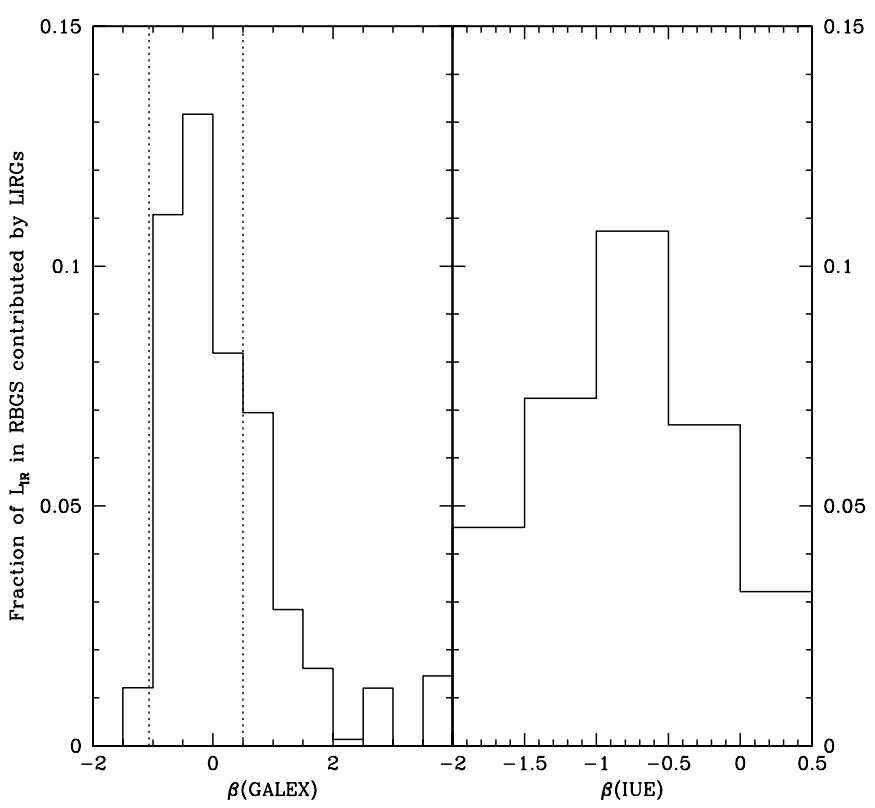

Figure 7. Fraction of total IR luminosity summed over all 629 systems in the RBGS sample contributed by LIRGs and ULIRGs with known UV colors (the GOALS GALEX sample, 112 systems). The IR luminosity fraction defined in this way is shown as a function of $\beta(G A L E X)$ (left panel) and $\beta(I U E)$ (right panel) over the range of the conversion given in the Appendix. The dotted lines in the left panel mark the range of $\beta(G A L E X)$ shown in the right panel. At least $19 \%$ of the IR luminosity of the RBGS is produced by (U)LIRGs with red UV colors $(\beta>0)$.

\subsection{Explaining Scatter in the IRX- $\beta(G A L E X)$ Relation}

The trend for certain populations to have, on average, larger values of IRX and redder values of $\beta(G A L E X)$ (parallel to the starburst relation) has been explained as a sequence in optical depth (Charlot \& Fall 2000). Thus, on average, more luminous LIRGs and ULIRGs have more extinction than less luminous LIRGs, and interacting systems have more extinction than noninteracting systems. This is consistent with the evolutionary scenario mentioned earlier.

We interpret the scatter of LIRGs and ULIRGs in the IRX- $\beta(G A L E X)$ diagram as follows. Deviations to the right of the starburst relation are interpreted as purely the result of redder UV colors (extra NUV emission for a given amount of FUV emission), most likely due to light from older stellar populations (Kong et al. 2004). Deviations above the starburst relation are interpreted as the result of increases in IRX, which we define as $\triangle I R X$. This quantity is a measure of the extent to which the IR and UV emission become decoupled, for example, in heavily obscured nuclei which emit strongly in the FIR (UV radiation reprocessed by dust) but do not contribute to the observed (escaping) UV emission. Like the starburst relation, $\triangle I R X$ is not necessarily accurate for $\beta(G A L E X)<-1$ or $\beta(G A L E X)>0.5$. A minimum of $11 \%$ of the total $L_{\mathrm{IR}}$ of the RBGS sample is produced in LIRGs and ULIRGs with $\triangle \mathrm{IRX}>1$, an order of magnitude above the starburst relation.

Cortese et al. (2006) concluded that attempting to estimate $L_{\mathrm{IR}}$ from rest-frame UV data of high-redshift galaxies will be uncertain by $>50 \%$ for normal galaxies. We find that using the starburst relation to estimate $L_{\mathrm{IR}}$ from rest-frame UV observations of LIRGs and ULIRGs would on average underestimate $L_{\mathrm{IR}}$ by a factor of 2.7 with a range of $L_{\mathrm{IR}}$ (true)/ $L_{\mathrm{IR}}$ (estimated) between 0.2 and 68 . Overestimates can be much greater for red UV colors beyond the range of the starburst 


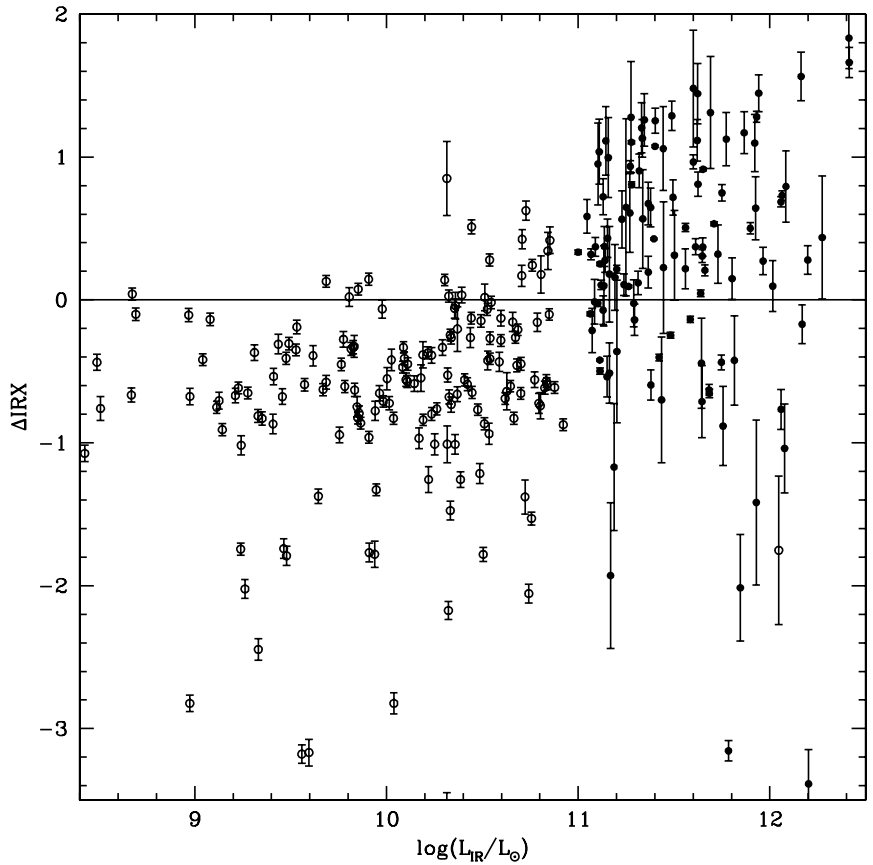

Figure 8. $\triangle \mathrm{IRX}$ vs. $L_{\mathrm{IR}}$. GOALS systems are shown as solid points, while galaxies from GDP are shown as open points. $\triangle \mathrm{IRX}$ increases with IR luminosity for $L_{\mathrm{IR}} \gtrsim 10^{10} L_{\odot}$.

relation $(\beta(G A L E X)>0.5)$, up to a factor of 2400 for a linear extrapolation. Previous studies have investigated possible second parameters for the scatter of normal galaxies to the right the starburst relation. Using a sample of a wide variety of galaxy types, Seibert et al. (2005) found no correlation between the deviation from the starburst relation and $L_{\mathrm{IR}}, L_{\mathrm{UV}}, L_{\mathrm{bol}}$, or UV and optical colors. Among normal galaxies, any correlation with star formation history is weak (Kong et al. 2004; Cortese et al. 2006) or nonexistent (Seibert et al. 2005; Boquien et al. 2009).

A number of observables might explain the scatter in $\Delta \mathrm{IRX}$, providing a second parameter to allow more accurate measurements of $L_{\mathrm{IR}}$ at high redshift as well as physical insight into the evolution of LIRGs and ULIRGs. A central question is what mechanism(s) lead to the UV emission being heavily obscured or decoupled from the IR emission in (U)LIRGs (Goldader et al. 2002) but not in lower luminosity starbursts? Since many LIRGs and essentially all ULIRGs are merger remnants with intense, compact, dust-enshrouded nuclear starbursts or AGN, a concentration parameter might correlate with IRX- $\beta(G A L E X)$ scatter as an indicator of decoupled IR and UV emission. Similarly, warm IR colors such as IRAS $25 \mu \mathrm{m} / 60 \mu \mathrm{m}$ might indicate dust in close proximity to a powerful UV source (starburst or AGN). AGNs provide another possible mechanism to explain scatter from the starburst relation. The [3.6]-[4.5] and [5.8]-[8] IRAC colors (Stern et al. 2005) can be used as an indicator of AGN emission. Systems identified as potential AGN might correlate with larger IRX above what the starburst relation would predict. Finally, although heightened IRX in a population of LIRGs and ULIRGs is most logically explained by elevated IR emission, it is possible for low UV emission to produce the same result.

As shown in Figure 8, $\Delta$ IRX increases with IR luminosity for $L_{\mathrm{IR}} \gtrsim 10^{10} L_{\odot}$, with considerable scatter. GOALS systems with IRAC colors that may indicate the presence of an AGN tend to have larger IRX ratios by a factor of 6 . No correlation is found between $\triangle \mathrm{IRX}$ and any of the following quantities:

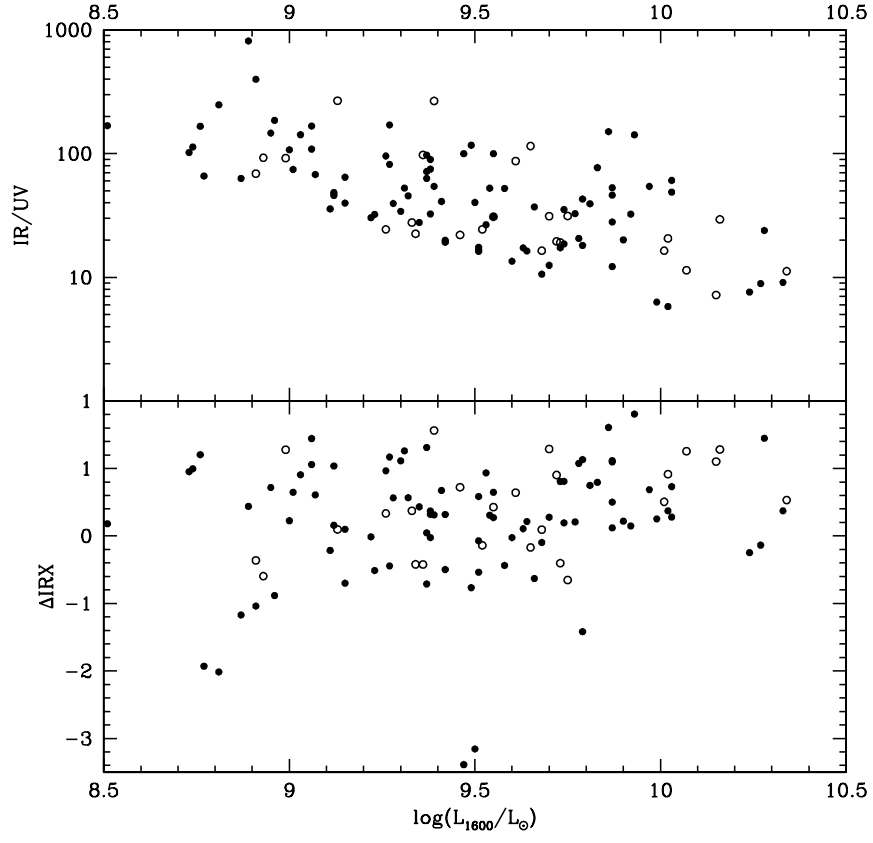

Figure 9. Top: the IR/UV ratio plotted against $L_{1600}$, the luminosity at $1600 \AA$ (interpolated from FUV and NUV). The lower envelope shows the sample selection cutoff of $L_{\mathrm{IR}}>10^{11} L_{\odot}$. Bottom: $\Delta \mathrm{IRX}$ vs. $L_{1600}$. No trend is seen; galaxies of high $\triangle I R X$ span the full range of UV luminosity. In both panels, galaxies with IRAC colors suggesting a significant AGN contribution are shown as open circles.

IRAS $25 \mu \mathrm{m} / 60 \mu \mathrm{m}$ color, IRAS $60 \mu \mathrm{m} / 100 \mu \mathrm{m}$ color, Spitzer $8 \mu \mathrm{m} / 24 \mu \mathrm{m}$ color, $L_{\mathrm{FUV}}, 8 \mu \mathrm{m}$ concentration $(1 \mathrm{kpc} /$ total $)$. The lack of correlation between $\Delta \mathrm{IRX}$ and global parameters other than $L_{\mathrm{IR}}$ suggests that the decoupling between UV and IR emission takes place on sub-kpc scales in most LIRGs and ULIRGs, well below our resolution with GALEX and Spitzer MIPS $24 \mu \mathrm{m}$, which is $2.6 \mathrm{kpc}\left(6^{\prime \prime}\right)$ at the median distance of the GOALS sample $(89 \mathrm{Mpc})$. Future studies (e.g., with Herschel and Hubble Space Telescope (HST)) at higher spatial resolution in the FIR and UV will be able to investigate this further. Such studies have already been done for a few nearby quiescent starforming galaxies. Boquien et al. (2009) found that variation in dust extinction curves and geometry is the most important factor determining the location of individual star-forming regions on the IRX- $\beta$ diagram. Muñoz-Mateos et al. (2009) examined radial profiles of all available SINGS galaxies and found that star formation history is the primary driver determining the position on the IRX $-\beta$ diagram of a radial annulus within a galaxy. The lack of correlation between $\triangle \mathrm{IRX}$ and FIR colors suggests that when dust is close to the heating source (producing warm FIR colors), that source is obscured and the UV color $\beta(G A L E X)$ increases along with IRX. Galaxies with positive $\triangle I R X$ span a range of $\log \left(L_{\mathrm{FUV}}\right)$ from 8.6 to 10.3 uniformly. The range of FUV luminosities indicates that LIRGs and ULIRGs with large $\triangle \mathrm{IRX}$ value are IR bright, not UV faint. Figure 9 shows the IR/UV ratio and $\triangle \mathrm{IRX}$ plotted against the $1600 \AA$ A luminosity (derived by linear interpolation between FUV and NUV).

In order to explore the dependence of IRX and $\beta$ on the morphological properties of LIRGs, all GOALS systems were visually classified as either interacting or non-interacting based on the inspection of the Spitzer IRAC 3.6 $\mu$ m images. A galaxy was deemed interacting if it exhibited a tidal bridge or tail, double nuclei, multiple galaxies in a common envelope, or a disturbed morphology. The interacting and non-interacting 


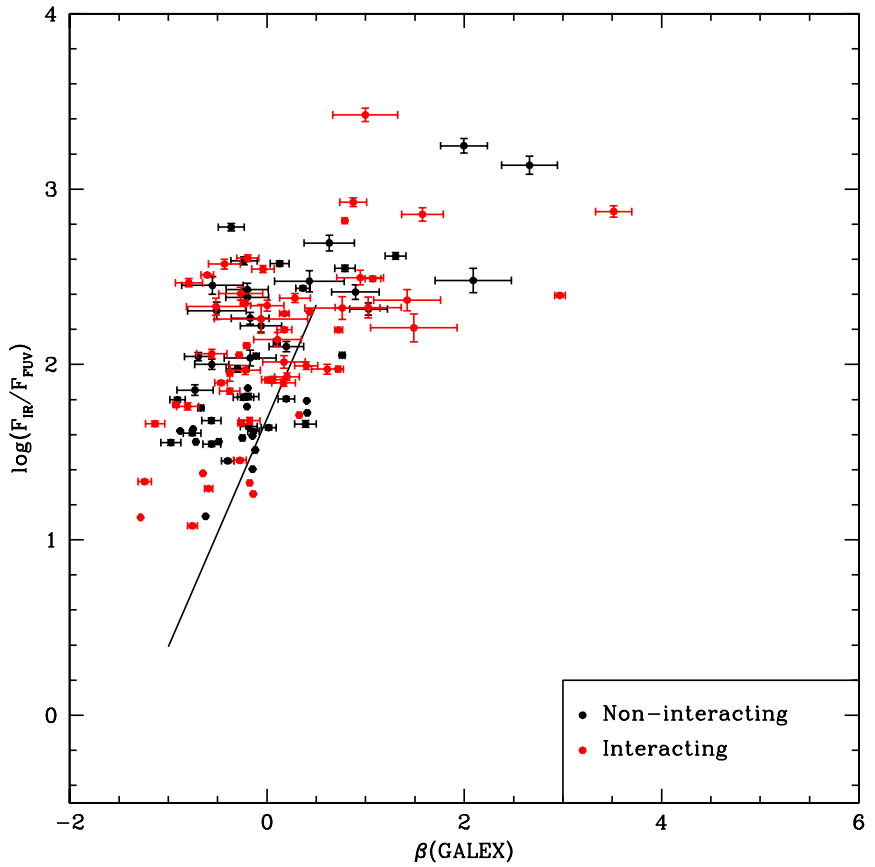

Figure 10. IRX- $\beta(G A L E X)$ plot comparing interacting (red) and noninteracting (black) LIRG systems. The solid line is the same as in Figure 1. The interacting and non-interacting populations are consistent with being drawn from the same distribution.

subpopulations are shown on the IRX- $\beta(G A L E X)$ diagram in Figure 10. Although the median position of the interacting population has redder $\beta$ (median 0.0 versus -0.19 ) and larger IRX (2.01 versus 1.86 ) than the non-interacting population, the two populations are consistent with being drawn from the same distribution. The galaxies with the lowest IRX are predominantly interacting, and these systems are among the most UV-luminous sources in the GOALS sample with $\log \left(L_{\mathrm{FUV}} / L_{\odot}\right) \gtrsim 10$.

UVLGs are an interesting type of object to compare with (U)LIRGs since they are objects with extremely high SFR but little dust obscuration. Five LIRGs in our sample are also UVLGs or near-UVLGs $\left(L_{\mathrm{FUV}} \geqslant 10^{10.2} L_{\odot}\right)$ : Arp 256 , VV 114 , Arp 240, NGC 6090, and CGCG 448-020. The stellar masses of these systems range from $11.1 \leqslant \log \left(M_{\text {stellar }} / M_{\odot}\right) \leqslant 11.5$. SFRs derived from the combination of UV and IR luminosities range from $1.8 \leqslant \log \left(\frac{\mathrm{SFR}}{M_{\odot} \mathrm{yr}^{-1}}\right) \leqslant 2.2$, and SSFRs range from $-9.6 \leqslant \log \left(\mathrm{SSFR} / \mathrm{yr}^{-1}\right) \leqslant-8.9$. The sample of Heckman et al. (2005) is divided into Large UVLGs and Compact UVLGs, which have mass ranges of $10.5 \leqslant \log \left(M_{\text {stellar }} / M_{\odot}\right) \leqslant 11.1$ and $9.5 \leqslant \log \left(M_{\text {stellar }} / M_{\odot}\right) \leqslant 10.7$, respectively, SFR ranges of $0.6 \leqslant \log \left(\frac{\mathrm{SFR}}{M_{\odot} \mathrm{yr}^{-1}}\right) \leqslant 1.2$ and $0.6 \leqslant \log \left(\frac{\mathrm{SFR}}{M_{\odot} \mathrm{yr}^{-1}}\right) \leqslant 1.4$, respectively, and SSFR ranges of $-10.5 \leqslant \log \left(\mathrm{SSFR} / \mathrm{yr}^{-1}\right) \leqslant$ -9.5 and $-9.8 \leqslant \log \left(\mathrm{SSFR} / \mathrm{yr}^{-1}\right) \leqslant-8.6$, respectively. The LIRG UVLGs have larger stellar masses and considerably higher SFR than either the Large or Compact UVLG samples as a whole. The LIRG UVLGs have similar SSFR to the Compact UVLG sample, the latter group being considered as local analogs to high-redshift Lyman break galaxies (LBGs; see Overzier et al. 2009).

\subsection{Optical and $U V$-selected (U)LIRG Samples}

Figures 3 and 4 show that, on average, (U)LIRGs with high SSFR have larger IRX and IR/UV and redder $\beta$ than (U)LIRGs with lower SSFR, implying greater extinction by dust in the high
SSFR systems. The GOALS sample spans the same range of SSFR as the UV-selected sample of Buat et al. (2009). However the UV-selected sample does not include galaxies with high IRX $(\log ($ IRX $) \gtrsim 2.0)$, which comprise $48 \%$ of the GOALS sample. The LIRGs in the LBG sample of Buat et al. (2009) include some systems similar to the GOALS UVLGs, while the majority have higher $L_{\mathrm{UV}}$ and low IRX.

The inverse of SSFR provides a doubling timescale for the stellar mass of a galaxy. The range for GOALS systems (excluding those with IRAC colors suggesting a possible AGN) is from $18 \mathrm{Gyr}$ to $290 \mathrm{Myr}$, with a median of 2.6 Gyr. Kaviraj (2009) fit double-burst star formation history models to a large sample of SDSS-selected LIRGs out to $z=0.2$, finding average burst ages of $7 \mathrm{Gyr}$ and $1 \mathrm{Gyr}$. The 43 systems in common between Kaviraj (2009) and the GOALS GALEX sample are consistent with being drawn from the same distribution in $\beta$ and IRX as the entire GOALS GALEX sample.

\subsection{Resolved Systems and Implications for Unresolved LIRGs at High Redshift}

As emphasized by Charmandaris et al. (2004), individual galaxies in interacting systems can have very different farinfrared and UV properties leading to incorrect assumptions about the system as a whole when viewed as a single unresolved system (e.g., at high redshift). In particular, these authors note that the mid-IR/UV ratios of the components of the Arp 299 and VV 114 systems vary by well over an order of magnitude between the individual interacting galaxies. Our combined GALEX and Spitzer observations of the GOALS sample show that this situation exists in a significant number of LIRG systems at low redshift. We define a source that produces at least twice as much luminosity as the companion to be dominant at that wavelength. Among LIRGs which can be resolved into interacting galaxies, approximately $32 \%$ consist of one galaxy which dominates the IR luminosity while a companion dominates the UV (hereafter referred to as "VV 114-like" systems). Extrapolating to number counts at $z \geqslant 1$ as in Charmandaris et al. (2004), this implies that as many as $15 \%-30 \%$ of high-redshift galaxies are unresolved VV 114-like systems.

In $21 \%$ of resolved systems, a single galaxy dominates both the IR and UV emission (such as Arp 182, for example). On average, the $\Delta \mathrm{IRX}$ value of the dominant galaxy is over four times larger than that of IR-dominant galaxies of similar UV color in a VV 114-like system. If we look at the masses of resolved pairs, the $\Delta \mathrm{IRX}$ of the more massive galaxy is on average four times greater than that of the less massive galaxy. These are independent effects: the IR-dominant galaxy in a resolved system is likely to dominate the mass of the system regardless of its contribution to the UV luminosity of the system. If we make the simplistic assumption that LIRG mergers form a single evolutionary sequence, our observations suggest that the phase in which the component galaxies have comparable IR and UV emission is $50 \%$ longer than the phase in which a single galaxy dominates both wavelengths. Furthermore, the fact that the high mass component is above the starburst relation would also be consistent with the fact that a synchronization of the nuclear starbursts in the two interacting galaxies is rare.

The ability to visualize merger simulations at observed wavelengths from the FUV to the FIR will facilitate the interpretation of data sets such as that presented in this paper. The SUNRISE code of Jonsson et al. (2006) may help answer outstanding questions such as: what types of mergers (and what 
fraction of viewing orientations) consist of an IR-dominant LIRG with a UV-dominant companion? What mergers consist of an LIRG which dominates both IR and UV relative to its companion? How long do these phases last? Do certain types of progenitor galaxies (Hubble type, mass ratio, gas fraction, orbit, etc.) lead to different observables (IRX, $\beta(G A L E X)$, IR or UV fraction, SSFR, etc.) during the merger?

Although the different definitions of $\beta(G A L E X)$ preclude a direct comparison, the GOALS sample appears to be generally consistent with the merger simulations shown in Jonsson et al. (2006). In particular, the ULIRG simulations predict an IRX that is up to a factor of 10 times greater than starburst galaxies with a narrow range of blue to intermediate UV colors. The GOALS ULIRGs within the same range of $\beta(I U E)$ have a median $\triangle I R X$ of 0.9 .

\section{CONCLUSIONS}

We present a comparison of the UV and infrared properties of 135 LIRGs and ULIRGs in the GOALS sample observed by GALEX and Spitzer. We find that

1. LIRGs have larger IR excesses than lower luminosity galaxies of similar UV color. On average, more luminous LIRGs and ULIRGs have larger IRX and redder colors.

2. The contribution of the FUV to the measured SFR is on average 4\%; UV emission alone is not a reliable indicator of the SFR for LIRGs.

3. The median SSFR of the GOALS sample $\left(3.9 \times 10^{-10} \mathrm{yr}^{-1}\right.$, corresponding to a mass doubling timescale of $2.6 \mathrm{Gyr})$ is approximately equal to the maximum SSFR seen in lower luminosity galaxies; however, the median IR/UV ratio (39) for GOALS galaxies is more than an order of magnitude greater.

4. Deviations from the starburst IRX- $\beta(G A L E X)$ relation $\triangle \mathrm{IRX}$ increase with IR luminosity for $L_{\mathrm{IR}} \gtrsim 10^{10} L_{\odot}$, with considerable scatter. LIRG systems with IRAC colors that may indicate the presence of an AGN have average IRX ratios a factor of 6 larger than the rest of the sample. $\triangle \mathrm{IRX}$ is not strongly correlated with IRAS $25 \mu \mathrm{m} / 60 \mu \mathrm{m}$ color, IRAS $60 \mu \mathrm{m} / 100 \mu \mathrm{m}$ color, Spitzer $8 \mu \mathrm{m} / 24 \mu \mathrm{m}$ color, $L_{\mathrm{FUV}}$, or $8 \mu \mathrm{m}$ concentration $(1 \mathrm{kpc} /$ total $)$.

5. A minimum of $19 \%$ of the total $L_{\mathrm{IR}}$ of the RBGS sample is produced in LIRGs and ULIRGs with $\beta>0$, sources that are typically absent from UV-selected samples at high redshift. A minimum of $11 \%$ of the total $L_{\mathrm{IR}}$ of the RBGS sample is produced in LIRGs and ULIRGs with $\Delta I R X>1$, an order of magnitude above the starburst relation.

6 . Using the starburst IRS $-\beta$ relation to estimate $L_{\mathrm{IR}}$ from rest-frame UV observations of LIRGs and ULIRGs would underestimate $L_{\mathrm{IR}}$ by a factor of 3 on average, with a wide range (factors of 0.2-68) of possible under- or overestimates, particularly for red UV colors (large values of $\beta$ ) where $L_{\mathrm{IR}}$ could be overestimated by as much as a factor of 2400 using a linear extrapolation of the starburst relation.

7. The UV and IR properties of GOALS systems are qualitatively consistent with an evolutionary picture in which some galaxies transition from LIRGs to ULIRGs over the course of a major merger event. More luminous galaxies, mergers, and galaxies with high SSFR are more heavily obscured than less luminous galaxies, non-mergers, and galaxies with lower SSFR.
8. Among LIRG systems resolved into individual interacting galaxies, pairs in which one galaxy dominates the IR emission while the companion dominates UV emission (such as the well-studied LIRG system VV 114) are more common than pairs in which one galaxy dominates both wavelengths (32\% and $21 \%$ of the sample, respectively). On average, galaxies which dominate both wavelengths have $\Delta I R X$ values four times larger than an IR-dominant galaxy in a "VV 114-like" system. The large fraction of "VV 114-like" systems has important implications for observations of interacting galaxies at high redshift in that the IR and UV properties of the unresolved systems can differ by over an order of magnitude from the properties of the component galaxies.

This research has made use of the NASA/IPAC Extragalactic Database (NED) which is operated by the Jet Propulsion Laboratory, California Institute of Technology, under contract with the National Aeronautics and Space Administration. This research has made use of the NASA/IPAC Infrared Science Archive, which is operated by the Jet Propulsion Laboratory, California Institute of Technology, under contract with the National Aeronautics and Space Administration. Based on observations made with the NASA Galaxy Evolution Explorer. GALEX is operated for NASA by the California Institute of Technology under NASA contract NAS5-98034. V.C. acknowledges partial support from the EU ToK grant 39965 and FP7-REGPOT 206469. We thank Ranga Chary, Brian Siana, and Harry Teplitz for helpful discussions. We thank Armando Gil de Paz for making his GALEX background subtraction code available, Danny Dale for providing the SINGS data points in Figure 3, and the anonymous referee for helpful comments.

\section{APPENDIX \\ UV COLORS}

The UV color of an object can be parameterized in several ways, complicating the comparison of results between different data sets. The UV continuum slope $\beta$ was defined by Calzetti et al. (1994) for use with IUE spectra. More recent photometric instruments such as STIS (Goldader et al. 2002) and GALEX cannot directly measure this spectroscopic $\beta$, referred to as $\beta(I U E)$ in the main text of this paper and in Figure 1. Instead, the slope between an NUV data point and an FUV data point is measured and labeled $\beta$, referred to as $\beta(G A L E X)$. Some authors abandon the UV slope and instead measure a conventional color FUV-NUV, expressed in magnitudes (e.g., GDP).

Since 11 galaxies from Meurer et al. (1999) are included in GDP, we derive an empirical conversion between $\beta(I U E)$ and $\beta(G A L E X)$ :

$$
\beta(I U E)=(-0.3 \pm 0.1)+(1.6 \pm 0.2) \beta(G A L E X) .
$$

This conversion is not necessarily valid outside the range $-2<\beta(I U E)<0.5$ or $-1<\beta(G A L E X)<0.5$.

\section{REFERENCES}

Armus, L., et al. 2009, PASP, 121, 559

Boquien, M., et al. 2009, ApJ, 706, 553

Buat, V., Takeuchi, T. T., Burgarella, D., Giovannoli, E., \& Murata, K. L. 2009, A\&A, 507, 693

Calzetti, D., Kinney, A. L., \& Storchi-Bergmann, T. 1994, ApJ, 429, 582 
Caputi, K. I., Dole, H., Lagache, G., McLure, R. J., Dunlop, J. S., Puget, J.-L., Le Floc'h, E., \& Pérez-González, P. G. 2006, A\&A, 454, 143

Charlot, S., \& Fall, S. M. 2000, ApJ, 539, 718

Charmandaris, V., Le Floc'h, E., \& Mirabel, I. F. 2004, ApJ, 600, L15

Charmandaris, V., Stacey, G. J., \& Gull, G. 2002, ApJ, 571, 282

Cortese, L., et al. 2006, ApJ, 637, 242

Dale, D. A., et al. 2007, ApJ, 655, 863

de Vaucouleurs, G., de Vaucouleurs, A., Corwin, H. G., Jr., Buta, R. J., Paturel, G., \& Fouque, P. 1991, Third Reference Catalogue of Bright Galaxies, Vols. $1-3$, XII (Berlin: Springer)

Genzel, R., Tacconi, L. J., Rigopoulou, D., Lutz, D., \& Tecza, M. 2001, ApJ, 563,527

Gil de Paz, A., et al. 2007, ApJS, 173, 185 (GDP)

Goldader, J. D., Meurer, G., Heckman, T. M., Seibert, M., Sanders, D. B., Calzetti, D., \& Steidel, C. C. 2002, ApJ, 568, 651

Heckman, T. M., et al. 2005, ApJ, 619, L35

Jonsson, P., Cox, T. J., Primack, J. R., \& Somerville, R. S. 2006, ApJ, 637, 255

Kaviraj, S. 2009, MNRAS, 394, 1167

Kennicutt, R. C., Jr. 1998, ARA\&A, 36, 189

Kennicutt, R. C., Jr., et al. 2003, PASP, 115, 928

Kong, X., Charlot, S., Brinchmann, J., \& Fall, S. M. 2004, MNRAS, 349, 769

Lacey, C. G., Baugh, C. M., Frenk, C. S., Silva, L., Granato, G. L., \& Bressan, A. 2008, MNRAS, 385, 1155
Le Floc'h, E., et al. 2005, ApJ, 632, 169

Leitherer, C., et al. 1999, ApJS, 123, 3

Magnelli, B., Elbaz, D., Chary, R. R., Dickinson, M., Le Borgne, D., Frayer, D. T., \& Willmer, C. N. A. 2009, A\&A, 496, 57

Magorrian, J., et al. 1998, AJ, 115, 2285

Meurer, G. R., Heckman, T. M., \& Calzetti, D. 1999, ApJ, 521, 64

Muñoz-Mateos, J. C., et al. 2009, ApJ, 701, 1965

Overzier, R. A., et al. 2009, ApJ, 706, 203

Salpeter, E. E. 1955, ApJ, 121, 161

Sanders, D. B., Mazzarella, J. M., Kim, D.-C., Surace, J. A., \& Soifer, B. T. 2003, AJ, 126, 1607

Sanders, D. B., \& Mirabel, I. F. 1996, ARA\&A, 34, 749

Sanders, D. B., Soifer, B. T., Elias, J. H., Madore, B. F., Matthews, K., Neugebauer, G., \& Scoville, N. Z. 1988a, ApJ, 325, 74

Sanders, D. B., Soifer, B. T., Elias, J. H., Neugebauer, G., \& Matthews, K. 1988b, ApJ, 328, L35

Seibert, M., et al. 2005, ApJ, 619, L55

Stern, D., et al. 2005, ApJ, 631, 163

Surace, J. A., \& Sanders, D. B. 2000, AJ, 120, 604

Surace, J. A., Sanders, D. B., \& Evans, A. S. 2000, ApJ, 529, 170

Tacconi, L. J., Genzel, R., Lutz, D., Rigopoulou, D., Baker, A. J., Iserlohe, C., \& Tecza, M. 2002, ApJ, 580, 73

Wyder, T. K., et al. 2005, ApJ, 619, L15 